\title{
Monte Carlo Modeling of
}

\section{Crystal Channeling at High Energies}

THIS IS A TEMPORARY TITLE PAGE

It will be replaced for the final print by a version

provided by the service academique.

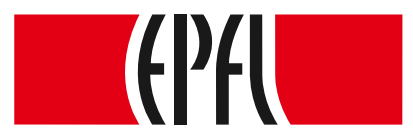

ÉCOLE POLYTECHNIQUE FÉDÉRALE DE LAUSANNE
Thèse n. 6046

présentée le 02 décembre 2013

à la Faculté des Sciences de Base, laboratoire LPAP

programme doctoral en Physique

École Polytechnique Fédérale de Lausanne

pour l'obtention du grade de Docteur ès Sciences

par

Philippe Schoofs

acceptée sur proposition du jury:

Prof. Frédéric Mila, président du jury

Prof. Leonid Rivkin, directeur de thèse

Dr. Francesco Cerutti, co-directeur de thèse

Dr. Mike Seidel, rapporteur

Dr. Stefano Redaelli, rapporteur

Dr. Chiara Bracco, rapporteur

Lausanne, EPFL, 2013 



\section{ACKNOWLEDGEMENTS}

A PhD cannot be fulfilled without helpful advices, discussions and exchanges of ideas. I would like to thank all of those with whom I have had the luck to interact during these years.

First of all, I would like to thank my supervisor Francesco Cerutti for his constant support. His extensive knowledge goes from particle-matter interaction to scientific English and I am glad to have had someone like him as supervisor. This would not have become a $\mathrm{PhD}$ topic without the help of Prof. Rivkin, who has given most precious advices and has always been kind and supportive throughout this work. My deepest thanks go to him as well. I also would like to thank Roberto Losito for giving me the opportunity to pursue my research in this very interesting field at the crossroads between solid-state physics, particle-matter interaction and Monte Carlo programing.

I would like to express my gratitude towards George Smirnov who has introduced me to the field of crystal channeling. Also, I would like to thank Alfredo Ferrari, always supportive of the work, and giving more than precious advices about multiple aspects of the method.

Then, I would like to extend these thanks to Walter Scandale and also to Simone Montesano who coordinates the experimental activities with great efficiency. But also Yury Gavrikov for the long nights spent in the North Hall during which he taught me a lot and Valentina Previtali, Daniele Mirarchi, Will Ferguson and Mark Pesaresi for much appreciated discussions about experimental setups, data acquisition and reconstruction, or aspects of the simulation.

Finally, I am grateful towards the friends that have been there for me in the last few years and especially Cedric with whom I have spent much time discussing our respective works and discovering this nice region. It made my time at CERN an even better experience. I also want to thank my colleagues from the EET section with a special "thank you" to Alessio, Lefteris and Thanasis for all the good times shared together. I could not end without underlining the support I got from my family back in Brussels, so much appreciated all along the work. 



\section{SUMMARY}

Charged particles entering a crystal close to some preferred direction can be trapped in the electromagnetic potential well existing between consecutive planes or strings of atoms. This channeling effect can be used to extract beam particles if the crystal is bent beforehand. Crystal channeling is becoming a reliable and efficient technique for collimating beams and removing halo particles. At the European Organization for Nuclear Research (CERN), the installation of silicon crystals in the Large Hadron Collider (LHC) is under scrutiny by the UA9 collaboration with the goal of investigating if they are a viable option for the collimation system upgrade. This thesis describes a new Monte Carlo model of planar channeling which has been developed from scratch in order to be implemented in the FLUKA code simulating particle transport and interactions. Crystal channels are described through the concept of continuous potential taking into account thermal motion of the lattice atoms and using Moliere screening function. The energy of the particle transverse motion determines whether or not it is trapped between the crystal planes while single Coulomb scattering on lattice atoms can lead to dechanneling. The volume capture and reflection applying to quasi-channeled particles are also modeled. Analogously to dechanneling, single scattering is used to determine the occurrence of volume capture. The parameters of the crystals, such as torsion or miscut, are described as well. For channeled particles, the suppression of electromagnetic and nuclear collisions is implemented. It is stronger for particles oscillating close to the center of the channel and is crucial for a correct evaluation of the rate of dechanneling without having recourse to the use of a macroscopic dechanneling length.

The UA9-H8 experiment conducted at CERN aims at investigating new crystal physics as well as characterizing crystals that can be of interest in view of the implementation of crystal collimation at CERN, including in the LHC. This experiment uses silicon strip detectors situated on both sides of the crystal. Putting together upstream and downstream tracks in coincidence and matching at an identical fitted location on the crystal, it yields information about the deflections given to the beam population. Several runs from the UA9-H8 experiment are analyzed and compared to the model results. Channeling and dechanneling rates, as well as angular distributions at crystal exit are shown to be in a very encouraging agreement both for strip and quasi-mosaic crystals.

Keywords : Crystal, channeling, Monte Carlo, Fluka, volume reflection, volume capture, Molière potential, crystal-assisted collimation 



\section{RÉSUMÉ}

Des particles chargées entrant dans un cristal avec une orientation proche de l'un des axes de symmetrie du réseau peut être piégée dans les canaux existant entre des plans ou chaînes d'atomes consécutives. Cet effet, dit de channeling, est utilisable dans un crystal courbé pour extraire les particles d'un faisceau. A l'Organisation Européenne pour la Recherche Nucléaire (CERN), l'installation de cristaux de silicium dans le Grand Collisionneur de Hadrons (Large Hadron Collider, LHC) fait l'objet de recherches afin de déterminer si elle est viable dans le cadre de l'amélioration du système de collimation. Cette thèse décrit un nouveau modèle Monte Carlo du channeling dans les cristaux qui a été développé en vue d'être inséré dans le code FLUKA qui simule le transport des particules et leur interaction avec la matière. Les canaux sont décrits au travers du concept de potentiel continu et prenant en compte le mouvement thermique des atomes. L'énergie du mouvement transverse de la particule détermine si elle est channelée ou pas. Les diffusions coulombiennes sur les atomes et électrons sont la source de dechanneling. Le traitement des effets de volume tels la reflection ou la capture des particules quasi-channelées est également inclus. De manière analogue au dechanneling, le scattering coulombien est également utilisé pour reproduire la capture volumique. D'autres propriétés des cristaux dont la torsion et le miscut peuvent également être pris en compte dans le modèle. L'alteration des modèles FLUKA existants permet de diminuer l'occurence des collisions electromagnétiques et nucléaires pour les particules en channeling, d'autant plus qu'elles oscillent près du centre du canal. Cette suppression permet d'obtenir un taux de dechanneling cohérent avec les prédictions, sans avoir recours à un paramètre macroscopique.

L'expérience UA9-H8 menée au CERN étudie la physique des cristaux et vise a caractériser ceux qui pourraient être utilisés dans un futur système de collimation par cristal, notamment dans le LHC. Cette expérience utilise 5 Silicon Strip Detectors situés de part et d'autre du cristal. En comparant les traces amont et aval obtenues en coïncidence et aboutissant aux mêmes positions d'impact sur le cristal, on obtient des traces composées permettant l'analyse des deflections angulaires dues au cristal. Plusieurs runs UA9-H8 ont été analysés et comparés aux résultats du modèle. Les taux de channeling et de dechanneling, ainsi que les distributions angulaires en sortie de cristal sont reproduites de manière très encourageantes par le modèle pour des cristaux de type strip et quasimosaique.

Mots clefs : Cristal, channeling, Monte-Carlo, Fluka, réflection volumique, capture volumique, potentiel de Molière, collimation par cristaux 



\section{LIST OF FIGURES}

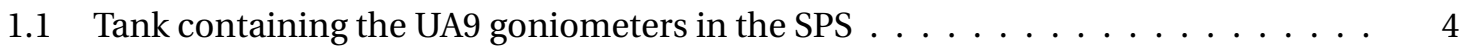

1.2 Standard and crystal-assisted collimation layouts $\ldots \ldots \ldots \ldots \ldots$

2.1 Conventional and primitive unit cells for body-centered cubic (BCC) and facecentered cubic $($ FCC) lattices. . . . . . . . . . . . . . . . . . 8

2.2 Examples of two nodes-based lattices : honeycomb and diamond-shaped lattices 9

2.3 (100), (110) and (111) orientations of the diamond-shaped lattice . . . . . . . . 10

2.4 Debye temperature and absolute temperature . . . . . . . . . . . . . . . . 12

2.5 Planar continuous potential and channel potential . . . . . . . . . . . . 14

2.6 Thermal variations of the channel potential . . . . . . . . . . . . . . . 15

2.7 Centrifugal term and effective potential $\ldots \ldots \ldots \ldots \ldots \ldots$

2.8 Harmonic approximation of the effective potential . . . . . . . . . . . . 17

2.9 Channeling and volume reflection seen in terms of transverse energy $\ldots \ldots \ldots$

2.10 Volume capture and dechanneling seen in terms of transverse energy $\ldots . . . . . \quad 19$

2.11 Beam distribution against horizontal kick and crystal orientation . . . . . . . . . 22

3.1 Principle of a complete crystal collimation simulation tool . . . . . . . . . . 26

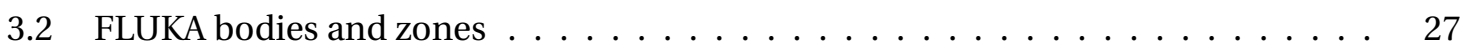

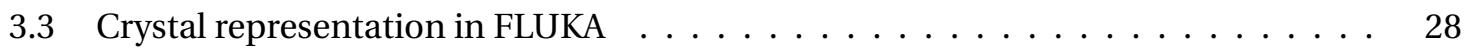

3.4 Crystal frame of reference vectors $\boldsymbol{A}$ and $\boldsymbol{B} \ldots \ldots \ldots \ldots$

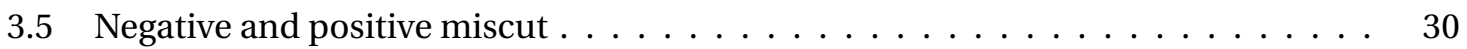

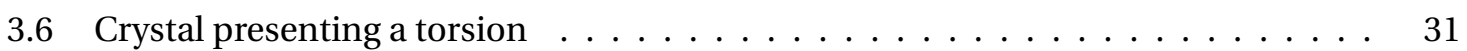

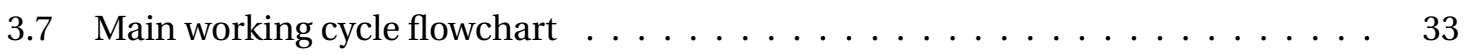

3.8 Volume reflection and volume capture $\ldots \ldots \ldots \ldots \ldots \ldots \ldots \ldots$

3.9 Quasi-channeled particles transverse energy spectrum . . . . . . . . . . . . . 37

3.10 Comparison of exit angles distributions $\ldots \ldots \ldots \ldots \ldots \ldots$

3.11 Minimum impact parameter for a channeled particle . . . . . . . . . . . . . 39

3.12 Form factor applied to Coulomb scattering of channeled particles . . . . . . . 40

3.13 Nuclear density across a channel . . . . . . . . . . . . . . . . . . . 42

3.14 Nuclear scaling factor across a channel . . . . . . . . . . . . . . . . . 43

3.15 Distribution of oscillation amplitudes for channeled protons . . . . . . . . 45

3.16 Moliere potential profile across a channel in three cases . . . . . . . . . . . 46

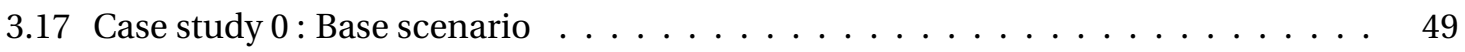

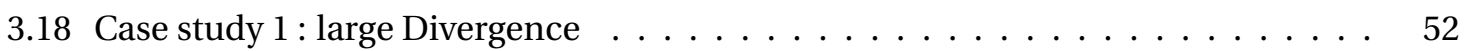

3.19 Case study 2 : with strong torsion $\ldots \ldots \ldots \ldots \ldots \ldots \ldots$

3.20 Case study $3:$ short crystal, small radius $\ldots \ldots \ldots \ldots \ldots \ldots$

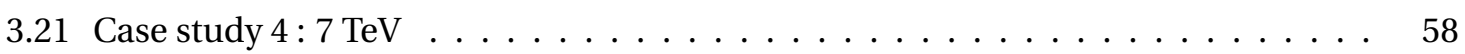

3.22 Case study $5:$ lead ions $\ldots \ldots \ldots \ldots \ldots \ldots \ldots$ 


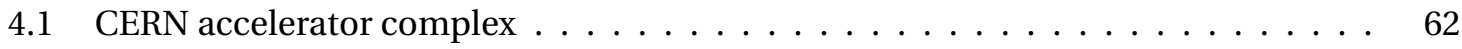

4.2 Septum extracting particles to the North Area. . . . . . . . . . . . . . . 63

4.3 Schematic layout of the TCC2 target area $\ldots \ldots \ldots \ldots \ldots \ldots \ldots$

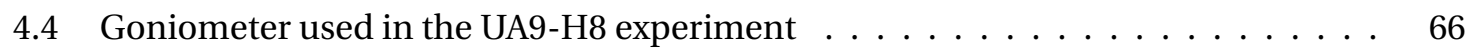

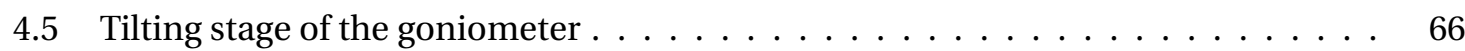

4.6 Strip and Quasi-Mosaic crystals $\ldots \ldots \ldots \ldots \ldots \ldots \ldots \ldots$

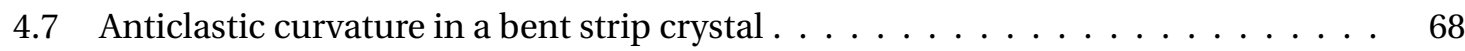

4.8 Quasi-mosaic curvature detailed . . . . . . . . . . . . . . . . . 69

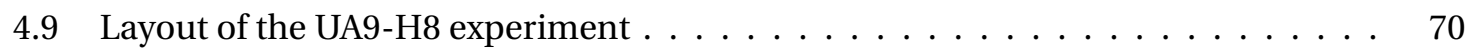

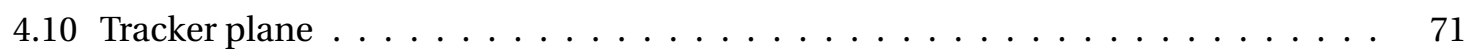

5.1 LHC strip - Incoming beam distributions $\ldots \ldots \ldots \ldots \ldots \ldots$

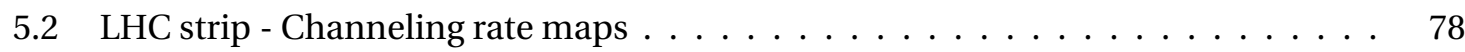

5.3 LHC strip - Angular deflection distribution . . . . . . . . . . . . . . . 79

5.4 LHC strip - Distribution of the incoming angles after torsion compensation . . . . 81

5.5 LHC strip - Channeling rate evolution along $x, y$ and $x^{\prime} \ldots \ldots \ldots$. . . . 82

5.6 LHC strip - Exit kick distribution after torsion compensation and narrow filter of

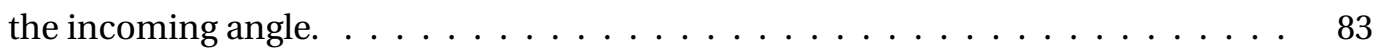

5.7 SPS strip - Channeling rate map as a function of $x^{\prime}$ and $y \ldots \ldots$. . . . . . 84

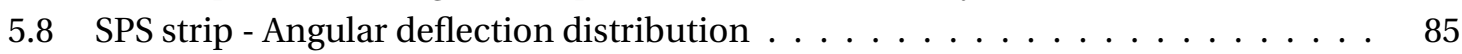

5.9 SPS strip - Channeling rate along $X, Y$ and $X^{\prime} \ldots \ldots \ldots \ldots \ldots \ldots$

5.10 SPS strip - Angular deflection distributions in VR orientation . . . . . . . . . . . 87

5.11 LHC QM - Angular deflection distributions $\ldots \ldots \ldots \ldots$

5.12 LHC QM - Channeling rate along $x, y$ and $x^{\prime} \ldots \ldots \ldots \ldots$

A.1 Flowchart of the event generator main function $\ldots \ldots \ldots \ldots$

A.2 Flowchart of the evaluation of the channeling status . . . . . . . . . . . . 102

A.3 Flowchart of the tracking in the volume effects zone . . . . . . . . . . . . . . 103

A.4 Flowchart of the transverse energy reevaluation after an interaction $\ldots \ldots \ldots$

B.1 Student distributions compared to the normal distribution . . . . . . . . . . . 108

B.2 Chi-square distributions . . . . . . . . . . . . . . . . . . . . . . 109

C.1 Run 608 - Angular deflection distributions . . . . . . . . . . . . . . . . . . 111

C.2 Run 630 - Angular deflection distributions . . . . . . . . . . . . . . . . . . . . 112

C.3 Run 899 - Angular deflection distributions . . . . . . . . . . . . . . . . . . . 112

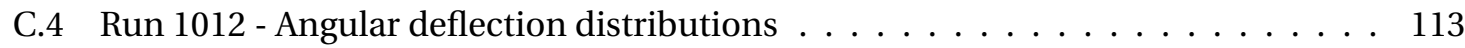

C.5 Run 1240 - Angular deflection distributions . . . . . . . . . . . . . . . . . . . . 114 


\section{LIST OF TABLES}

2.1 Channel widths in various types of Silicon channels $\ldots \ldots \ldots \ldots$

3.1 Transverse energy thresholds at crystal entrance $\ldots \ldots \ldots \ldots . \ldots \ldots$

3.2 Nuclear reaction suppression factors $\ldots \ldots \ldots \ldots \ldots \ldots \ldots \ldots$

3.3 Input parameters used in the case study $\ldots \ldots \ldots \ldots \ldots \ldots \ldots \ldots$

4.1 Technical data of the four goniometer stages $\ldots \ldots \ldots$. . . . . . . . . . 67

4.2 Characteristics of crystals tested in the UA9-H8 experiment $\ldots \ldots \ldots \ldots \ldots$

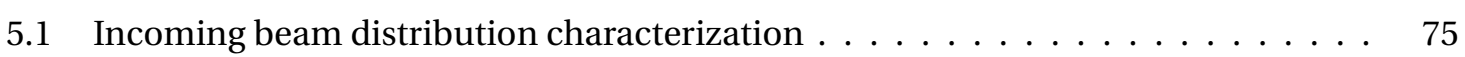

5.2 LHC strip - Key figures of the deflection distribution . . . . . . . . . . . . . . 80

5.3 SPS strip - Key figures of the deflection distribution $\ldots \ldots \ldots$. . . . . . . . . 85

5.4 SPS strip - Key figures of the deflection distribution in VR orientation . . . . . . . . 87

5.5 LHC QM - Key figures of the deflection distribution . . . . . . . . . . . . . 90

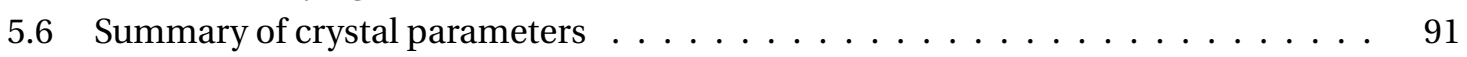

5.7 Channeling and reflected/amorphous peak characteristics from experiment and

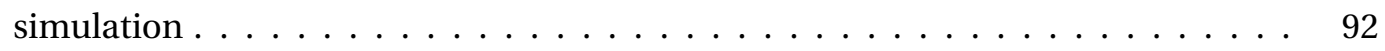

5.8 Results of the analysis for channeling and dechanneling rates $\ldots \ldots \ldots \ldots$ 



\section{ACRONYMS}
AM amorphous mode
BCC body-centered cubic
BLM beam loss monitor

CERN European Organization for Nuclear Research

CH channeled

Cl confidence interval

DAQ data acquisition system

EM electromagnetic

FCC face-centered cubic

IHEP Institute for High-Energy Physics, Protvino

LHC Large Hadron Collider

MCS multiple Coulomb scattering

NIR nuclear interaction rate

QC quasi-channeled

QM quasi-mosaic

rms root-mean square

SPS Super Proton Synchrotron

VC volume capture

VR volume reflection 



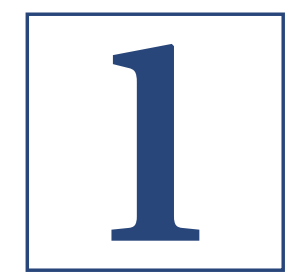

\section{FIRST CHAPTER}

\section{INTRODUCTION}

The Large Hadron Collider (LHC), the world's largest collider, has come to its first long shutdown period and the staff at the European Organization for Nuclear Research (CERN) are now working on its consolidation in order to approach its nominal energy. Later on, a significant luminosity upgrade is already planned. In view of this upgrade, improvement of the collimation system is under investigation, and crystals are amongst the tools under scrutiny in that context. The ability of bent crystals to deflect particles, equivalent to what would be the one of a several hundreds tesla magnet, can be used to clean the beam halo more efficiently than with standard amorphous collimators. As a function of the crystal channels orientation with respect to the beam direction, a fraction of the incident particles is "channeled", getting an angular kick and being removed from the beam halo, with a very limited impact on the crystal itself and a significant reduction of the secondary halo.

Since the sixties and Lindhard's description of a channel potential through the continuous approximation, up until the large number of experiments that have been carried out along the last years, crystal channeling has become a promising technique for collimating beams and raised quite some interest at accelerator centers around the world. In addition to CERN, it has been investigated at IHEP and at the Fermilab's Tevatron. However, no simulation tool gives a complete description of the beam and secondaries tracking in crystals and their surroundings yet, giving birth to this $\mathrm{PhD}$ topic.

Channeling occurs when particles entering the crystal with a specific angle are trapped inside natural channels formed by successive planes or strings of atoms. If the crystal is bent beforehand, this phenomenon can be used to deflect particles for collimation or extraction purposes. The continuous potential model assumes that every particle present in a channel is in the electromagnetic (EM) field of many lattice atoms and that 
the atomic potential can be averaged over a whole atomic plane or string. In the case of planar channeling, further approximating the continuous potential with a harmonic one leads to the equation of motion yielding a sinusoidal trajectory between the planes. In the transverse direction, oscillations are only limited by the atomic potential around the trajectory so that particles with a high transverse energy escape the channel and propagate in amorphous mode (AM) in the remainder of the crystal.

The main aim of this PhD activity was to develop an implementation of anisotropic crystal effects suitable for integration in the FLUKA Monte Carlo code, in order to include them in a simulation tool already of current use for accelerator design and upgrade, and machine protection purposes. The goal was to produce an event generator model able to describe in a general way all the physical processes occurring in crystals such as channeling, dechanneling, volume capture (VC) and volume reflection (VR).

A strictly related objective was the validation of the model with single-pass data in view of more demanding benchmarking along the Super Proton Synchrotron (SPS) ring. The H8 experiment from which the sets of data are taken is situated in CERN North experimental area, where particles are tracked by a telescope composed of 5 multi-strip detector planes on both sides of a crystal determining incoming and outgoing angles as well as hit positions. It is intended to provide several observables among which the characterization of the channeled and amorphous/reflected peak and the (de)channeling and VC rates.

In the course of this thesis, we will first address channeling-related phenomena and describe their original Monte Carlo implementation. Chapter 2 introduces relevant crystal structures ; their different orientations are presented and the respective channels are shown. Then, the effective potential inside a channel is derived, starting from the continuous potential formalism introduced by Lindhard and incorporating the Moliere screening function, as well as the thermal motion. Finally, the case of bent crystals, useful for collimation purposes, is introduced and all coherent processes taking place in crystals are addressed.

In chapter 3, the Monte Carlo event generator is described. First, the two frames of reference used to define the crystal position and orientation are introduced as well as the channels direction. Then, the course of the chapter describes the different stages of a particle travel across a crystal. It begins with its entry and the determination of its status (channeling, quasi-channeling or amorphous). It then describes oscillations and volume effects and, following this, the suppression of EM and nuclear interactions is discussed. Finally, a comparison of the model results is presented for different physics cases.

The second part of the thesis concerns the experimental activity providing the data used for benchmarking purposes. Chapter 4 focuses on the experiment conducted in 
CERN North Area on the H8 beamline. Its goal is to assess crystal performances in view of their possible installation in the SPS, where the collaboration is conducting a multi-pass crystal collimation experiment, or in the LHC, where a future crystal-assisted collimation scheme is under study in the framework of the new LUA9 project. Crystals types and fabrication process are discussed. Goniometer and detectors specifications are given as well.

Chapter 5 contains benchmarking studies, in which data from UA9-H8 experimental runs has been analyzed and compared with the model, spanning various beam and crystal parameters. Results concern the reproduction of channeling and dechanneling rates, and the distribution of angular deflection at the crystal exit. Runs from 2010 to 2012 involving strip and quasi-mosaic crystals were considered. In view of these results, possible additions to the model refining the event generator are discussed at the end of the chapter.

Finally, chapter 6 presents conclusions along with a summary of the most prominent results. It closes with an overview of possible improvements of the model for its broader application.

\section{Crystal Collimation}

The viability of crystal-assisted collimation as part of the LHC upgrade plan is being investigated by the UA9 collaboration in close collaboration with the CERN EN-STI group, for example in the SPS (fig. 1.1) where an experiment is ongoing since 2009. In a multistage collimation system, crystals have demonstrated a good level of performance. The results obtained by UA9 in the SPS showed that a short SPS-suited crystal creates 6 times less losses and a less populated far off-momentum halo which is the main source of collimation leakage $[1,2]^{1}$.As a consequence, the LUA9 experiment, investigating their use in the LHC, is being prepared at the time of writing this report, aiming to be run in 2015. This section briefly introduces the concept and the challenges associated with the use of coherent effects occurring in crystals towards beam cleaning.

The current multi-stage collimation system, described extensively in [3] for example, relies on nuclear scattering to clean the beam halo. As sketched in fig.1.2, a primary collimator (made of $60 \mathrm{~cm}$ of graphite) is placed as limiting aperture in the beam halo. As the inelastic scattering length in graphite is about $41 \mathrm{~cm}$ at $7 \mathrm{TeV}$, the majority of incoming protons interact in the collimator producing a cascade. The secondary collimator is designed to absorb these secondaries (secondary halo) preventing them from being lost

\footnotetext{
${ }^{1}$ For ions, the collimation efficiency, directly related to the channeling efficiency, was found to be large, even though some reserves are made concerning crystal resistance to radiation in a LHC ion beam.
} 


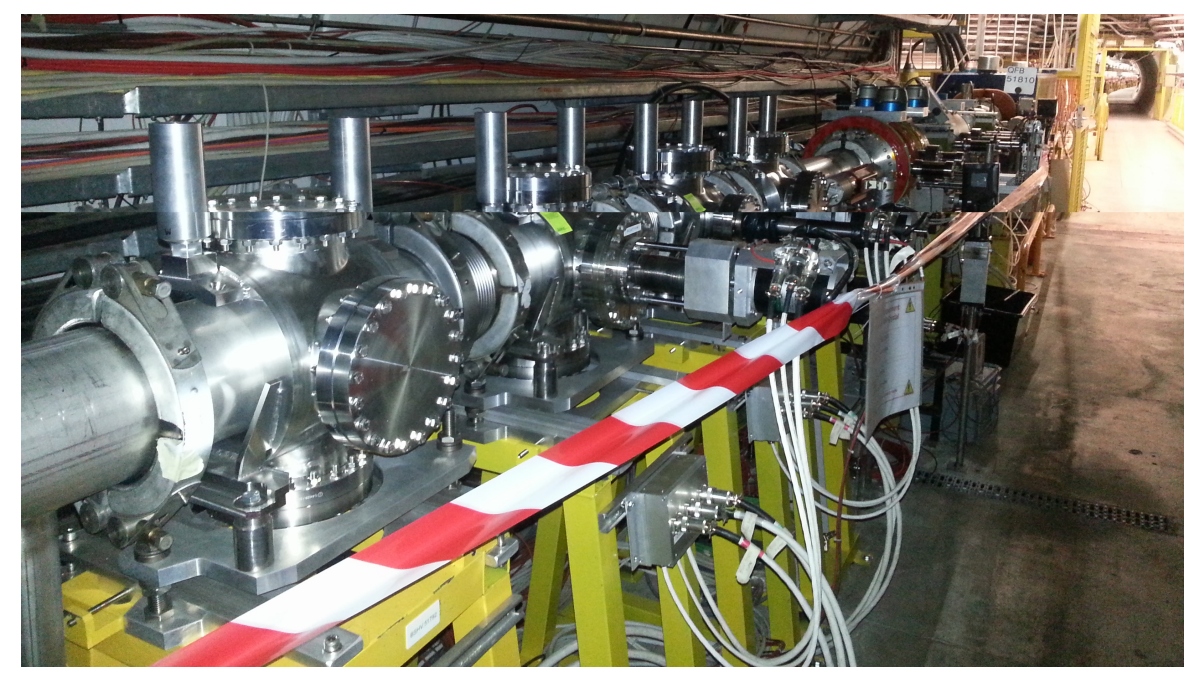

Fig. 1.1. Experimental setup of the UA9-SPS experiment : steel tanks containing the goniometers and the crystals. Their motion controllers can be seen on the right-hand side of the tanks. Courtesy A. Mereghetti.

in critical areas of the machine (cold magnets for example). However, another fraction of particles undergoing less destructive events (such as multiple Coulomb scattering (MCS) or single diffractive scattering) in the primary collimator is susceptible to get lost outside of the collimation region. So tertiary collimators are used to protect specific equipments against losses far from the collimation region.

Crystal collimation uses a bent crystal as primary collimator. Through channeling or volume reflection, a crystal can deflect coherently a fraction of the beam. As the crystal is typically a few $\mathrm{mm}_{\text {long }}{ }^{2}$, the number of nuclear interactions taking place inside of it is much lower than in a standard layout, and the secondary collimator has to deal essentially with primary particles. Its role becomes then different as it has to absorb primary beam particles instead of mainly secondaries, and additional shielding is required to deal with the cascade produced in turn in this absorber. But the strong reduction of nuclear interactions also means a strong decrease in single diffractive events. These events yield beam particles with slightly modified energy and angle that can be lost outside the collimation region and are the main contributor to far losses. Decreasing the amount of such interactions will lead to a reduction of the losses in critical parts of the ring ${ }^{3}$. On the other hand, the average amount of passages that a particle needs before it gets channeled depends on the channeling efficiency of the considered crystal. Some beam particles might need multiple passages before being trapped in a channel, tempering this effect. Finally, dechanneled particles escaping the crystal after having

\footnotetext{
2 which has the additional advantage of reducing the impedance budget of LHC of about $20 \%$ [4].

3 assuming that the interactions induced in the downstream absorber are not impacting the collimation efficiency
} 

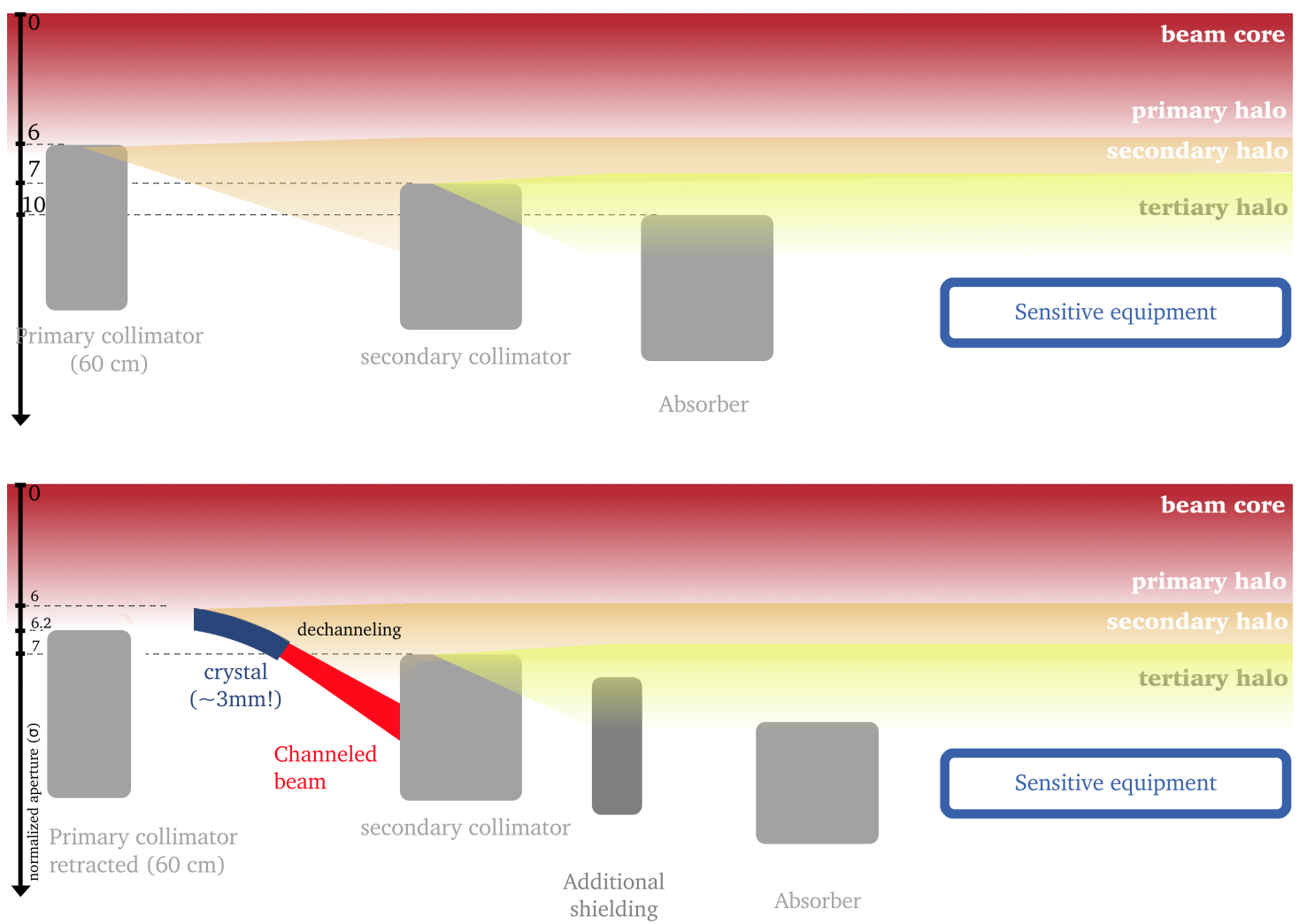

Fig. 1.2. Top : schematic view of the current collimation layout in which a majority of the beam particles undergoes a nuclear interaction, producing a cascade which is in turn absorbed by the secondary collimator. Tertiary collimators or absorbers protect specific equipments along the ring. Bottom : crystalassisted layout currently under investigation in the LHC. In a crystal-assisted setup, the channeled beam, consisting of primary particles, impacts the secondary collimator. The cascade coming out of the secondary will most likely require some additional shielding, playing the role of the current TCLA.

received a partial kick are likely to be lost outside the collimation region as well, which has to be taken into account in the design stage [5].

In the LHC, a crystal collimation experiment has been approved and is being installed in IR7 (betatron cleaning insertion) at the time of writing the report. There, crystals are to be installed on state-of-the-art goniometers providing accuracy and repeatability down to the $\mu \mathrm{rad}$ scale as, at $7 \mathrm{TeV}$, the angular aperture of the channels is about $2 \mu \mathrm{rad}$. Along with the placement of the crystal in perfect channeling orientation in a beam as energetic as the LHC, the design of absorbers able to handle a power density of the order of $500 \mathrm{~kW}^{4}[6]$ impacting on a region of a few square millimeters is the main challenge for the LUA9 project. Thermal and radiation loads present in the LHC could

\footnotetext{
${ }^{4}$ The nominal power density that the collimation system must be able to withstand is $100 \mathrm{~kW}$. During the design, an accidental scenario was considered in which $500 \mathrm{~kW}$ must be handled for $10 \mathrm{~s}$ before the beam is automatically dumped.
} 
prevent coherent effects by damaging the crystal structure and even affect the integrity of the device. In order to investigate the silicon crystal robustness, it has been put to the test in the HiRadMat facility, where a considerable fraction of the $440-\mathrm{GeV}$ proton beam, having a transverse size $\sigma=0.5 \mathrm{~mm}$, (and amounting in total to $10^{14}$ impacting particles) has hit the device without leaving any trace of macroscopic damage [7]. 


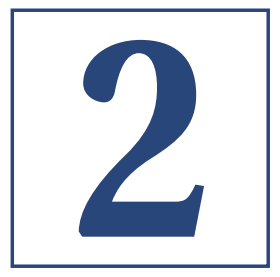

\section{SECOND CHAPTER}

\section{CRYSTAL CHANNELING BASICS}

In this chapter, basic notions of crystallography are introduced and the channels found along some preferred directions in crystals are described. The channel potential playing a major role in the channeling process is then derived. This allows to determine the channeling condition for a given incoming particle and to describe the effects taking place in crystals such as dechanneling, volume capture or volume reflection.

\subsection{Crystal Structure}

\subsection{1 | Bravais lattice}

This short excerpt of crystallography gives an overview of the main concepts useful to understand the geometry of channels inside a crystal. The reader looking for a more thorough description can refer to Solid-state physics handbooks, such as [8] for example. Crystals consist of a periodic arrangement of atoms. Given the primitive vectors $\boldsymbol{a}_{\boldsymbol{i}}$, $i=1,2,3$, a Bravais lattice can be defined as a finite or infinite ensemble of points whose coordinates are obtained through the following translation operation :

$$
\boldsymbol{T}=n_{1} \boldsymbol{a}_{\mathbf{1}}+n_{2} \boldsymbol{a}_{2}+n_{3} \boldsymbol{a}_{\mathbf{3}}
$$

where $n_{i} \leq N_{i}$ is the $i$-th index of the corresponding lattice node ${ }^{1}$.

The primitive cell is defined as the zone of space covering all space without overlapping or leaving voids when translated according to eq. (2.1) for all $n_{i}$. The more general unit cell covers the whole space only for a subset of the translations. The difference between the conventional and the primitive unit cell is emphasized in fig. 2.1 in the case of

\footnotetext{
${ }^{1}$ The number of nodes in the lattice being given by $N=N_{1} N_{2} N_{3}$.
} 


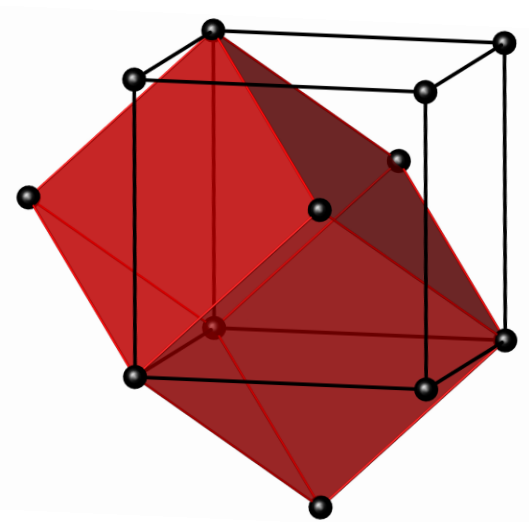

(a)

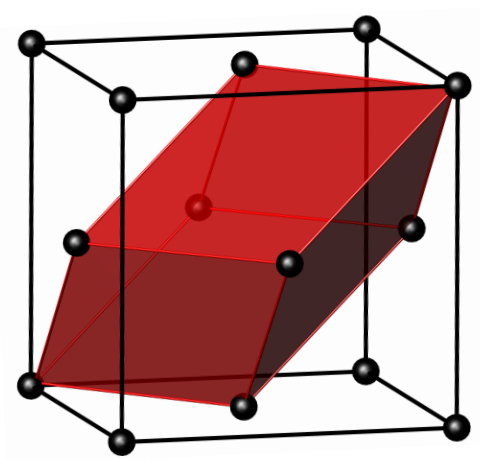

(b)

Fig. 2.1. Conventional unit cells (black) and primitive unit cells (red) for (a) a body-centered lattice and (b) a face-centered lattice [9].

a body-centered cubic (BCC) (left-hand side) and of a face-centered cubic (FCC) (righthand side) lattice. As a direct consequence of its definition, the primitive cell contains only one atom : in the example of fig. 2.1, 8 corner atoms contribute each for one eighth (in average because the cell does not necessarily have a cuboid shape).

The notion of Bravais lattice can be extended to take into account crystals with any arrangement of atoms, called a basis, inside their primitive cell. This arrangement, reproduced through the translation operation (2.1), gives birth to a lattice where all atoms are not necessarily equivalent ${ }^{2}$. Fig. 2.2 illustrates two examples of lattices with a basis containing two nodes. On the left, the two-dimensional honeycomb lattice is shown. Its atoms are of two types with different lattice layouts around them (one being the mirror-image of the other). Similarly, the diamond-structure (fig. 2.2,b) is composed of two FCC lattices, one being shifted by one quarter of the body diagonal. The primitive cell is the same as for the regular FCC lattice but contains 2 atoms : 8 eighths in the corners of the cell and 1 inside. The diamond structure is of particular interest in the context of this work. Indeed, the vast majority of crystals used in the context of crystal collimation are made of silicon or germanium, which follow that structure.

\subsection{2 | Channels orientation}

In crystals, directions are indicated through their Miller index $\langle i j k>$. This index represents the vector :

$$
<i j k>=i \boldsymbol{a}_{1}+j \boldsymbol{a}_{2}+k \boldsymbol{a}_{3}
$$

\footnotetext{
${ }^{2}$ That lattice is not a Bravais lattice per se because lattice nodes are not equivalent; they are not situated in the lattice in the same configuration.
} 


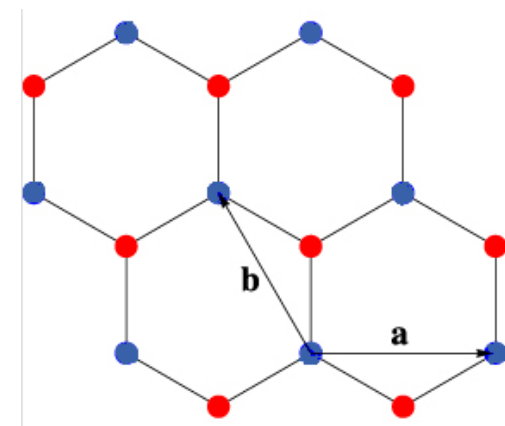

(a)

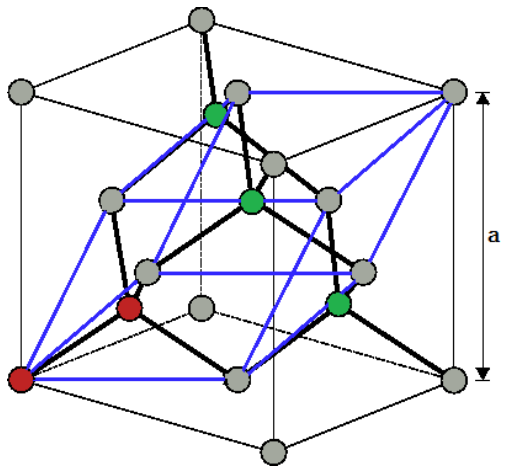

(b)

Fig. 2.2. Examples of two nodes-based lattices. (a) Honeycomb lattice with color emphasizing two kinds of atoms. (b) Diamond lattice composed of two intertwined FCC lattices (gray and green). Basis atoms are shown in red and an example of primitive cell is shown in blue.

Table 2.1. Channel widths in pure Silicon for the three main channel orientations. $(111)_{n}$ and $(111)_{w}$ refer respectively to the narrow and wide channels found along (111)-oriented silicon.

\begin{tabular}{c|cc}
\hline CHANnEL ORIENTATION & \multicolumn{2}{|c}{ CHANNEL WidTH } \\
\hline$(100)$ & $a / 4$ & $1.36 \AA$ \\
$(110)$ & $a / 2 \sqrt{2}$ & $1.92 \AA$ \\
$(111)_{n}$ & $a \sqrt{3} / 4$ & $0.78 \AA$ \\
$(111)_{w}$ & $3 a \sqrt{3} / 4$ & $2.35 \AA$ \\
\hline
\end{tabular}

where $\left(\boldsymbol{a}_{1}, \boldsymbol{a}_{2}, \boldsymbol{a}_{3}\right)$ are the lattice basis vectors. Similarly, we can define a family of parallel planes $(i j k)$ via their orthogonal vector $\langle i j k>$.

Channeling is possible when atoms are positioned in such a way that the EM potential of their screened nuclei creates canals in which charged particles can be confined. Depending on the symmetry of the confinement space, axial channeling or planar channeling may occur. In axial channeling, the atomic strings $\langle i j k\rangle$ form the channels while, in planar channeling, a family of parallel planes $(i j k)$ plays that role.

Fig. 2.3 gives an illustration of the three main orientations for planar channeling in a diamond-shaped lattice : from left to right, the (100), the (110) and the (111) orientations. The width of the channels is given in table 2.1 as a function of the lattice constant $a$ (equal to $5.43 \AA$ in Silicon) and in absolute terms. One can notice that, in the (111) orientation, channel planes are actually double and include 2 layers of atoms. This is not an effect of perspective: in reality there are two separated planes forming a second narrower channel. 


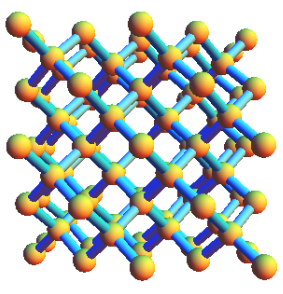

(a)

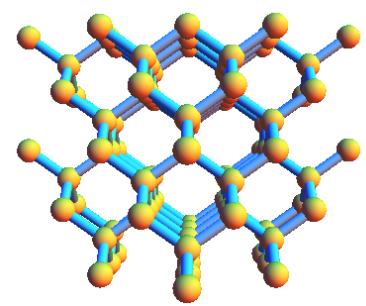

(b)

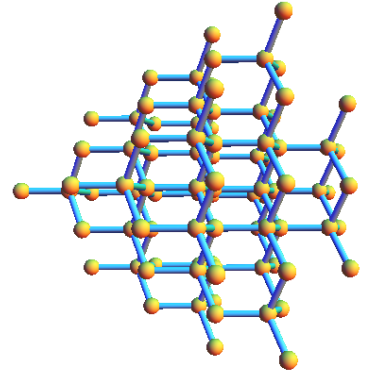

(c)

Fig. 2.3. Diamond-shaped lattice seen from the (a) (100) orientation, (b) (110) orientation and (c) (111) orientation. In the (100) and (110) orientations, there is only one family of planes, all equivalent. In consequence, all channels are the same. In the (111) orientation, the groupings actually host two different planes, forming two different channels (noted $(111)_{w}$ and $(111)_{n}$ ). The corresponding channel widths are given in table 2.1 .

\subsection{Potential in a channel}

In this section, the EM potential of a planar channel is derived. First, the continuous potential formalism describing the interaction of a particle with an entire plane of atoms, is introduced. Then, the modification of the continuous potential due to thermal motion is taken into account to find the expression of the straight planar potential. Finally, the case of a bent crystal, crucial to any collimation application, is considered with the centrifugal component of the potential introducing asymmetry and the notion of critical radius.

\subsection{1 | Continuous potential}

When a charged particle passes through a crystal close to one of its major symmetry axis or parallel to main atomic planes, it has a significant probability of undergoing a small-angle scattering. The probability that subsequent scatterings involve small-angles too is still high and, as a consequence, the particle will undergo a correlated series of collisions that constitutes the basis of the channeling effect.

Consider now a particle of charge $Z_{p}$ coming at a distance $r$ of a lattice atom of charge $Z_{c}$. The potential due to the lattice atom, taking into account the screening effect, is [10] :

$$
V(r)=\frac{Z_{p} Z_{c} e^{2}}{r} \Phi\left(\frac{r}{a_{t f}}\right)
$$


The Thomas-Fermi screening length $a_{t f}$ is given by [11] :

$$
a_{t f}=\left(\frac{9 \pi^{2}}{128}\right)^{1 / 3} a_{0}\left(Z_{c}^{2 / 3}+Z_{p}^{2 / 3}\right)^{-1 / 2}
$$

$a_{0}$ being the Bohr radius. In eq. (2.3), $\Phi$ is the screening function representing the distribution of the electron density in the atom. In the following, we use Molière's approximation for $\Phi[12]$ :

$$
\Phi\left(\frac{r}{a_{t f}}\right)=\sum_{i=1}^{3} \alpha_{i} \exp \left(-\frac{\beta_{i} r}{a_{t f}}\right)
$$

For particles coming towards a lattice preferred direction at small angles, successive scatterings cannot be considered independent events. This is the founding principle of the continuous potential theory developed in the 1960s by Lindhard [11]. The interaction potential of such incoming particles with a string or a plane of atoms is obtained through summation of the potential of the individual lattice nodes. The incoming angle being small, one can consider the potential from a continuous source and, in the case of planar channeling, the continuous form $U_{p l}$ is given by :

$$
U_{p l}(x)=N d_{p} \iint_{-\infty}^{\infty} V(x, y, z) \mathrm{d} y \mathrm{~d} z
$$

$N$ being the atomic density and $d_{p}$ the interplanar distance. Developing (2.6) using Molière screening function (2.5) in (2.3) :

$$
U_{p l}(x)=2 \pi N d_{p} Z_{c} Z_{p} e^{2} a_{t f} \sum_{i=1}^{3} \frac{\alpha_{i}}{\beta_{i}} \exp \left(-\frac{\beta_{i} x}{a_{t f}}\right)
$$

where $\left\{\alpha_{i}\right\}=\{0.1,0.55,0.35\}$ and $\left\{\beta_{i}\right\}=\{6.0,1.2,0.3\}$ for $i=\{1,2,3\}$ respectively.

The continuous potential prevents positively charged particles from approaching the atoms, thus reducing the amount of close-range interactions they undergo. The channeling of negatively charged particles is slightly different since they are confined in the neighborhood of the atoms and not in the space between them. They interact more than positively charged channeled particles and observed dechanneling lengths are in consequence much smaller. The work done in this thesis focuses on channeling of positively charged particles. The direct application of this work to experiments carried out at CERN with protons and lead ions justifies this choice.

\subsection{2 | Thermal motion}

The potential (2.7) is altered by the fact the lattice atoms are not static but have thermal motion. The mean square amplitude of this oscillation is given by [13] :

$$
u_{T}^{2}=\frac{\hbar^{2}}{M K \Theta_{D}}\left[1+4 \frac{T}{\Theta_{D}} \Gamma\left(\frac{\Theta_{D}}{T}\right)\right]
$$




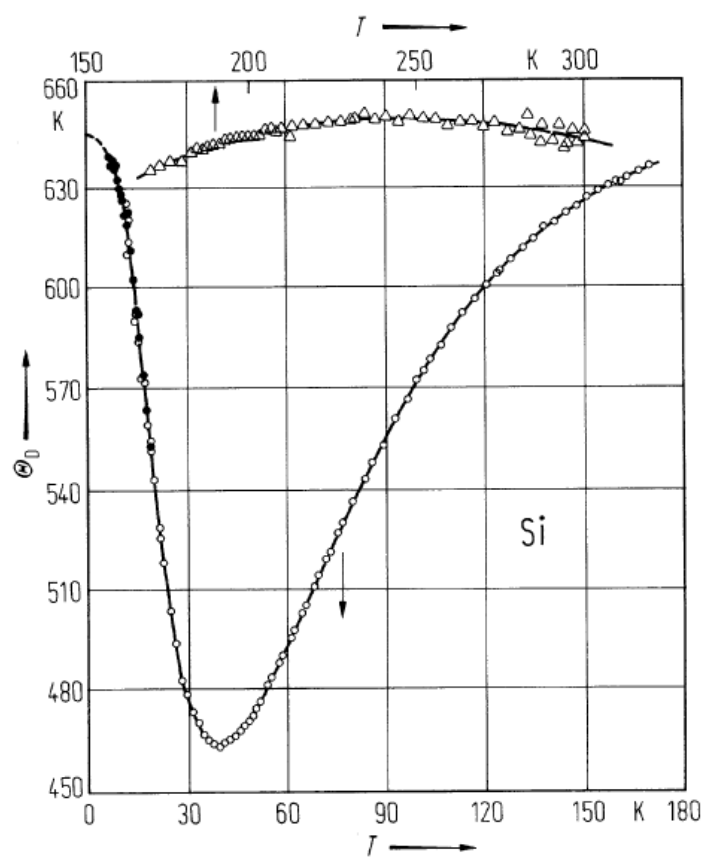

Fig. 2.4. Evolution of the Debye temperature $\Theta_{D}$ in Silicon as a function of the absolute temperature $T$. The bottom curve stands for temperature 0 to $170 \mathrm{~K}$ (bottom scale). The top curve applies for temperatures from 170 to $300 \mathrm{~K}$ (top scale) [14].

where $M$ is the atomic mass of crystal atoms, $K$ is the Boltzmann constant, $\Theta_{D}$ is the Debye temperature at the absolute temperature $T$ and

$$
\Gamma\left(\frac{\Theta_{D}}{T}\right)=\frac{T}{\Theta_{D}} \int_{0}^{\Theta_{D} / T} \frac{t}{e^{t}-1} \mathrm{~d} t
$$

The evolution of $\Theta_{D}$ as a function of the absolute temperature is shown in fig. 2.4. For the sake of simplicity, we will consider it takes the constant value of $640 \mathrm{~K}$, keeping in mind that this approximation is valid only for temperatures ranging from $150 \mathrm{~K}$ up to about $350 \mathrm{~K}$. At room temperature, we find that $u_{T}=0.075 \AA$.

Considering that the oscillations of the lattice atoms are independent from each other, the distribution of their transverse position follows the Gaussian distribution

$$
P_{T}(x)=\frac{1}{\sqrt{2 \pi u_{T}^{2}}} \exp \left(-\frac{x^{2}}{2 u_{T}^{2}}\right)
$$

In order to obtain the potential from the plane of oscillating atoms, consider a particle at a transverse coordinate $x$. The potential at that location is the sum of the contributions $V\left(\left|x-x^{\prime}\right|\right)$ from all the infinitesimal atomic layers $\left(x^{\prime}, x^{\prime}+\mathrm{d} x^{\prime}\right)$. We obtain the following 
convolution product :

$$
U_{p l, T}(x)=\int_{\infty}^{-\infty} V\left(\left|x-x^{\prime}\right|\right) P_{T}\left(x^{\prime}\right) \mathrm{d} x^{\prime}
$$

which yields in turn :

$$
\begin{aligned}
U_{p l, T}(x)= & \frac{K}{\sqrt{2 \pi u_{T}^{2}}} \sum_{i=1}^{3} \frac{\alpha_{i}}{\beta_{i}} \int_{\infty}^{-\infty} \exp \left(-\frac{\beta_{i}\left|x^{\prime}-x\right|}{a_{t f}}\right) \exp \left(-\frac{x^{\prime 2}}{2 u_{T}^{2}}\right) \mathrm{d} x^{\prime} \\
= & \frac{K}{\sqrt{2 \pi u_{T}^{2}}} \sum_{i=1}^{3} \frac{\alpha_{i}}{\beta_{i}}[\exp \left(-\frac{\beta_{i} x}{a_{t f}}\right) \underbrace{\int_{-\infty}^{x} \exp \left(\frac{\beta_{i} x^{\prime}}{a_{t f}}-\frac{x^{\prime 2}}{2 u_{T}^{2}}\right) \mathrm{d} x^{\prime}}_{A} \\
& +\exp \left(\frac{\beta_{i} x}{a_{t f}}\right) \underbrace{\int_{x}^{\infty} \exp \left(-\frac{\beta_{i} x^{\prime}}{a_{t f}}-\frac{x^{\prime 2}}{2 u_{T}^{2}}\right) \mathrm{d} x^{\prime}}_{B}]
\end{aligned}
$$

where $K=2 \pi N d_{p} Z_{c} Z_{p} e^{2} a_{t f}$. One can evaluate the integrals $A$ and $B$ using the substitution $t=\frac{x^{\prime}}{\sqrt{2} u_{T}}-\frac{\beta_{i} u_{T}}{\sqrt{2} a_{t f}}$ :

$$
\begin{aligned}
A & =\int_{-\infty}^{x} \exp \left[-\left(\frac{x^{\prime}}{\sqrt{2} u_{T}}-\frac{\beta_{i} u_{T}}{\sqrt{2} a_{t f}}\right)^{2}+\frac{\beta_{i}^{2} u_{T}^{2}}{2 a_{t f}^{2}}\right] \mathrm{d} x^{\prime} \\
& =u_{T} \sqrt{\frac{\pi}{2}} \exp \left(\frac{\beta_{i}^{2} u_{T}^{2}}{2 a_{t f}^{2}}\right)\left[1+\operatorname{erf}\left(\frac{x}{\sqrt{2} u_{T}}-\frac{\beta_{i} u_{T}}{\sqrt{2} a_{t f}}\right)\right]
\end{aligned}
$$

where

$$
\operatorname{erf}(x)=\frac{2}{\sqrt{\pi}} \int_{0}^{x} \exp \left(-t^{2}\right) \mathrm{d} t
$$

is the usual error function. From there comes :

$$
A=u_{T} \sqrt{\frac{\pi}{2}} \exp \left(\frac{\beta_{i}^{2} u_{T}^{2}}{2 a_{t f}^{2}}\right)\left[\operatorname{erfc}\left(\frac{\beta_{i} u_{T}}{\sqrt{2} a_{t f}}-\frac{x}{\sqrt{2} u_{T}}\right)\right]
$$

using one of the properties of the complementary error function $\operatorname{erfc}(x)$ :

$$
1+\operatorname{erf}(x)=\operatorname{erfc}(-x)
$$

In a similar way, one obtains for $B$ :

$$
B=u_{T} \sqrt{\frac{\pi}{2}} \exp \left(\frac{\beta_{i}^{2} u_{T}^{2}}{2 a_{t f}^{2}}\right)\left[\operatorname{erfc}\left(\frac{\beta_{i} u_{T}}{\sqrt{2} a_{t f}}+\frac{x}{\sqrt{2} u_{T}}\right)\right]
$$




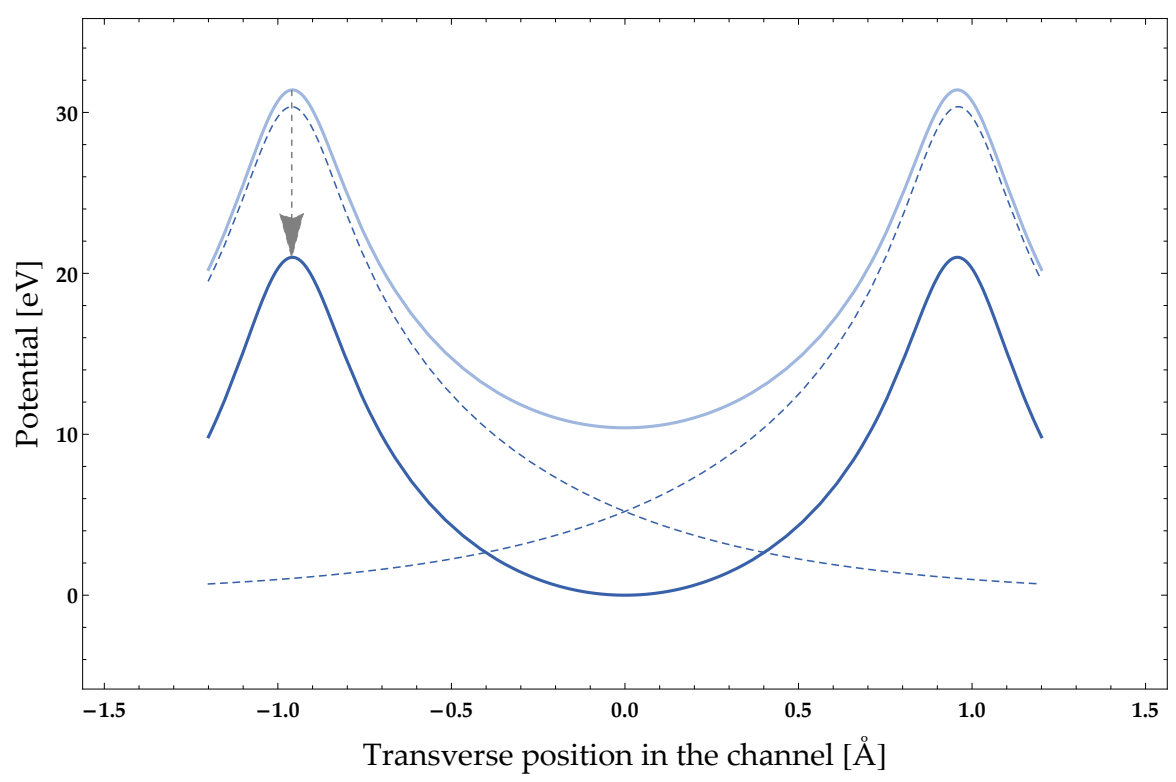

Fig. 2.5. The planar, continuous potentials for adjacent (110) planes in Silicon (dashed lines - see eq. (2.18)) and the straight crystal potential obtained from their addition (thin solid line). To better evaluate the potential barrier in the channel, one prefers to have the minimum of the potential curve at 0 (thick line, see eq. (2.19) )

Incorporating eqs. (2.15) and (2.17) into (2.12), we get for the planar potential :

$$
\begin{aligned}
U_{p l, T}(x) & =2 \pi N d_{p} Z_{c} Z_{p} e^{2} a_{t f} \\
& \times \sum_{i=1}^{3} \frac{\alpha_{i}}{2 \beta_{i}} \exp \left(\frac{\beta_{i}^{2} u_{T}^{2}}{2 a_{t f}^{2}}\right)\left\{\exp \left(-\frac{\beta_{i} x}{a_{t f}}\right) \operatorname{erfc}\left[\frac{1}{\sqrt{2}}\left(\frac{\beta_{i} u_{T}}{a_{t f}}-\frac{x}{u_{T}}\right)\right]\right. \\
& \left.+\exp \left(\frac{\beta_{i} x}{a_{t f}}\right) \operatorname{erfc}\left[\frac{1}{\sqrt{2}}\left(\frac{\beta_{i} u_{T}}{a_{t f}}+\frac{x}{u_{T}}\right)\right]\right\}
\end{aligned}
$$

The interplanar potential in a straight crystal $U_{s}$ is obtained by summing the contributions from the adjacent planes. Assuming $x=0$ at the center of the channel, we have :

$$
U_{s, T}(x)=U_{p l, T}\left(x-d_{p} / 2\right)+U_{p l, T}\left(x+d_{p} / 2\right)-2 U_{p l, T}(0) .
$$

where the last term reflects the choice

$$
U_{s, T}(0)=0 .
$$

Along with $U_{s, T}(x)$ (thick solid line), fig. 2.5 displays the planar potential from the two neighboring planes $U_{p l, T}(x)$ (dashed), their sum (thin solid) and the shift added to comply with (2.20). 


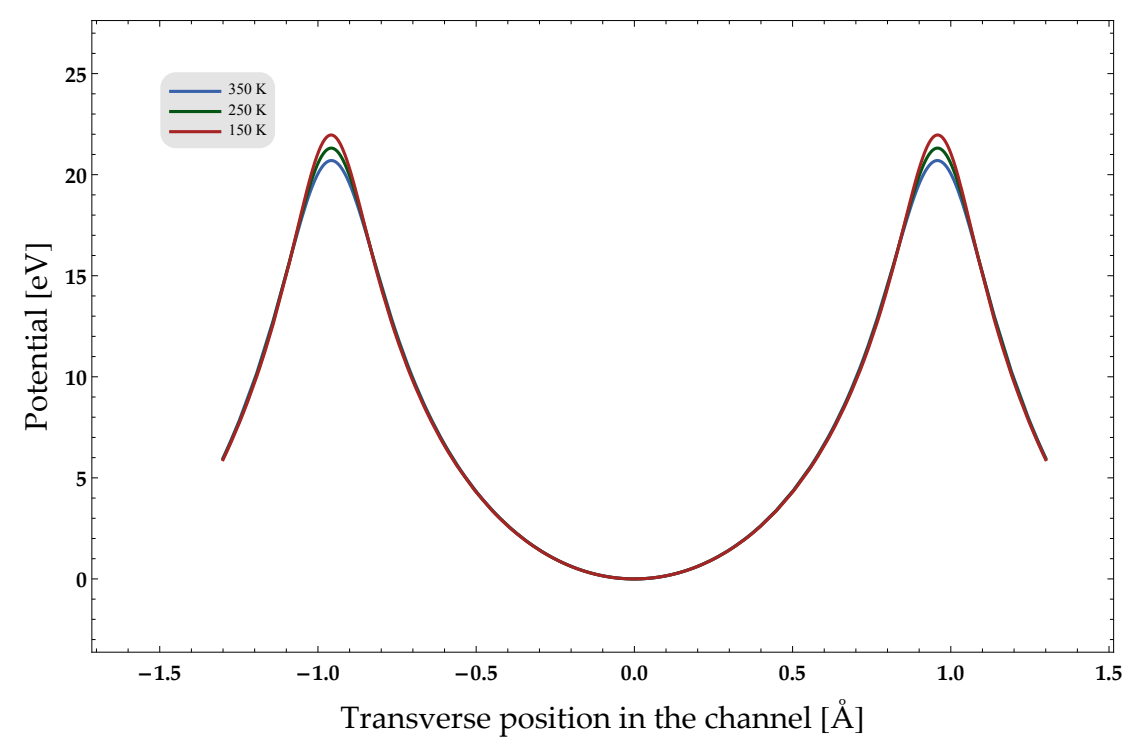

Fig. 2.6. Potential in a straight $<110>$ Silicon channel at different temperatures. The crystal Debye temperature is supposed constant at $640 \mathrm{~K}$. This assumption is acceptable for absolute temperatures ranging from $150 \mathrm{~K}$ to $350 \mathrm{~K}$.

The distribution (2.10) applies in the direction perpendicular to the channeling planes. The individual atomic displacements cause the potential maximum close to the channel boundary to become lower. This is illustrated in fig. 2.6, showing the continuous potential in a straight (110)-oriented Silicon channel for different temperatures compatible with the chosen constant value of $\Theta_{D}$. At higher temperatures, crystal atoms will have larger thermal motion amplitudes and produce a decrease in the potential barrier, slightly reducing the crystal ability to channel.

\subsubsection{In a bent crystal}

Crystals can be bent in order to give a transverse kick to channeled particles. The bending introduces a centrifugal term to the potential, linear in $x$. This results in an increase of the potential on the inner side of the bend and a decrease on the outer side. The effective potential, obtained by adding the centrifugal term to $U_{s, T}$ is given by :

$$
U_{e, T}(x)=U_{s, T}(x)+\frac{p v}{R} x
$$

where $p$ and $v$ are the particle momentum and velocity, respectively, and $R$ is the crystal radius of curvature. The effective potential is illustrated in fig. 2.7 and the reduction in the potential well depth appears clearly, the numerical value of which is indicated by the thin gray line. When the curvature of the crystal is augmented, the barrier gets shallower up to a point where it disappears. The radius of curvature corresponding to such a situation is the critical radius . 


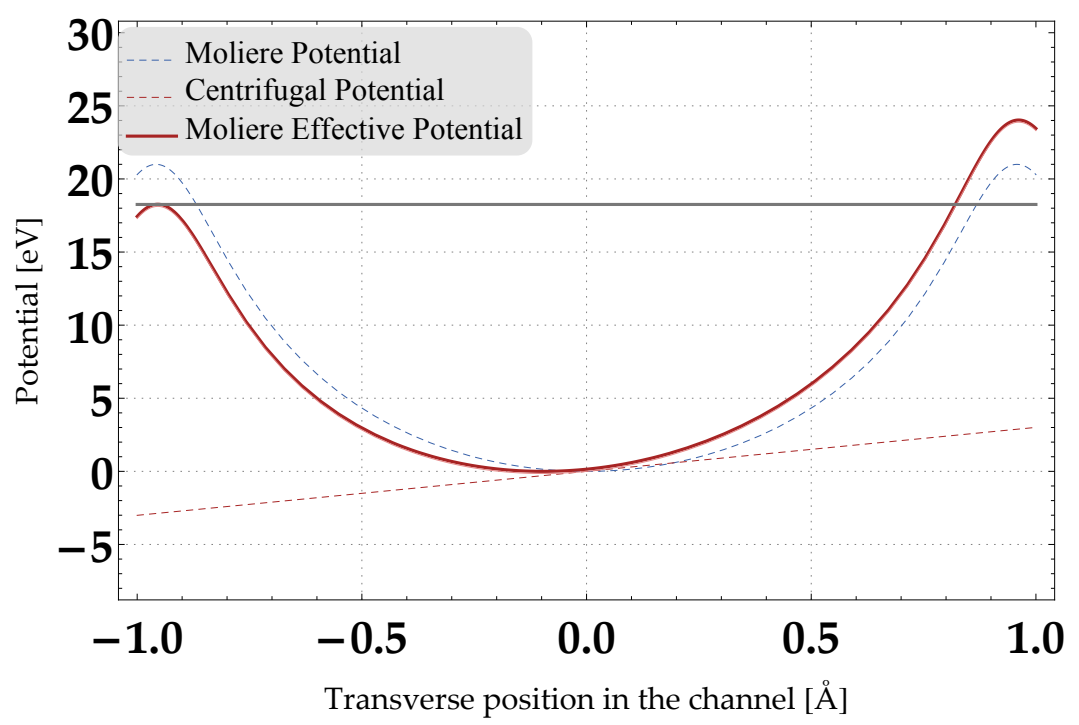

Fig. 2.7. Centrifugal potential component in a bent Silicon crystal having $R=6.5 \mathrm{~m}$ (red dashed line). Added to $U_{s, T}$ (blue dashed curve), it yields the effective potential $U_{e}$ given by eq. (2.21) (thin red solid line). The thick red line is obtained by shifting $U_{e}$ in order to comply with (2.20). Finally, the gray line indicates the numerical value of the potential barrier.

\subsection{Coherent behavior in a crystal}

\subsection{1 | Transverse Energy}

Consider a particle with $p_{x} \ll p_{z}$ and moving at an angle $\theta_{x} \approx p_{x} / p_{z}$. For the transverse motion (not relativistic, unlike the longitudinal one), the energy conservation leads to [10] :

$$
\begin{array}{r}
\frac{p_{x}^{2}}{2 m}+U_{e, T}(x)=\mathrm{CST} \\
\frac{p_{z}^{2} c^{2}}{2 E} \theta_{x}^{2}+U_{e, T}(x)=\mathrm{CST}
\end{array}
$$

where $E$ is the particle energy and $m=\gamma m_{0}$ is the mass of the particle (with $\gamma$ as the usual Lorentz factor). Making the approximation $p_{z} \approx p$ and using the relation $p c^{2}=v E$, (2.23) becomes :

$$
E_{x}=\frac{p v}{2} \theta_{x}^{2}+U_{e, T}(x)=\mathrm{CST} .
$$




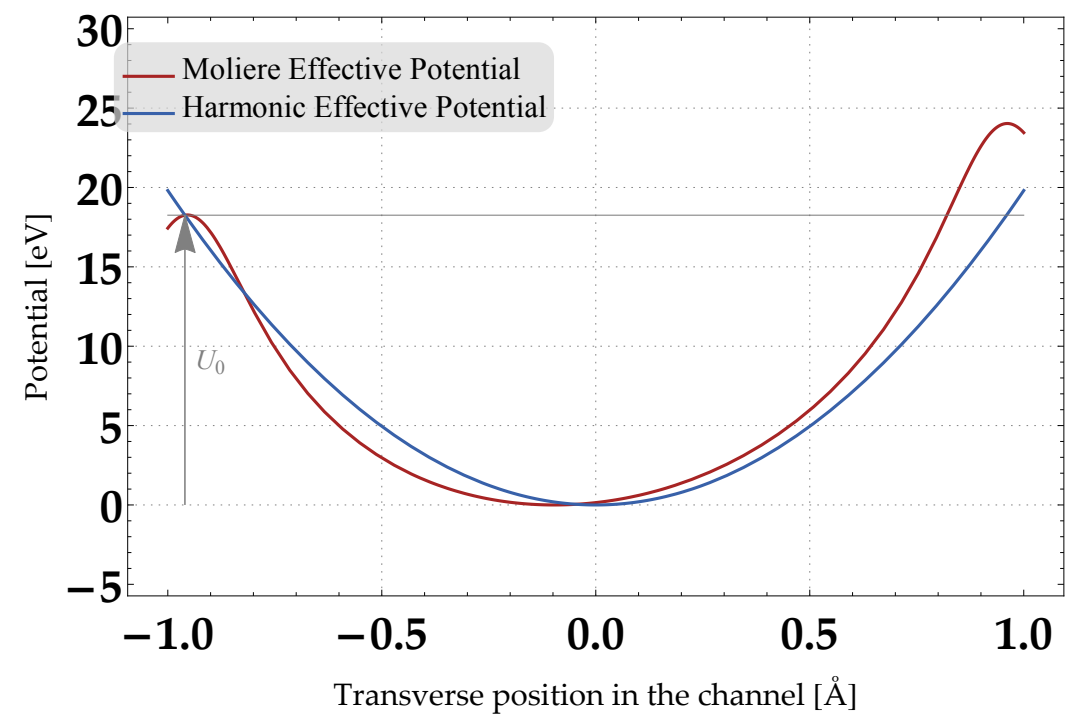

Fig. 2.8. Harmonic approximation (blue) of the effective potential from fig. 2.7 (red) having the same value at the channel lower edge.

\subsection{2 | Classical approach and harmonic approximation}

Let us now approximate the potential $U_{e, T}$ described above with the harmonic potential

$$
U_{h}(x)=\frac{1}{2} k x^{2}
$$

Assuming $U_{h}(x)$ takes the same value at the lower edge of the channel, $k$ is given by

$$
k=\frac{8 U_{0}}{d_{p}^{2}}
$$

where $U_{0}$ stands for $U_{e, T}\left(-d_{p} / 2\right)$, keeping the dependence on the temperature implicit. The quantum energy levels in the harmonic oscillator system described by $U_{h}(x)$ are separated by a quantity :

$$
\Delta E=\frac{\hbar}{d_{p}} \sqrt{\frac{8 U_{0}}{m}}
$$

and the number of levels in the potential well is :

$$
N_{l}=\frac{d_{p}}{\hbar} \sqrt{\frac{m U_{0}}{8}}
$$

For $400 \mathrm{GeV} / \mathrm{c}$ protons and a typical (110)-oriented crystal $15-\mathrm{eV}$ barrier, $N_{l} \approx 750$. The classical approach can be used with a good accuracy when $N_{l} \gg 1$ which is the case for most high energy accelerators applications $\left(N_{l}\right.$ scales with $\left.\sqrt{\gamma}\right)$. 


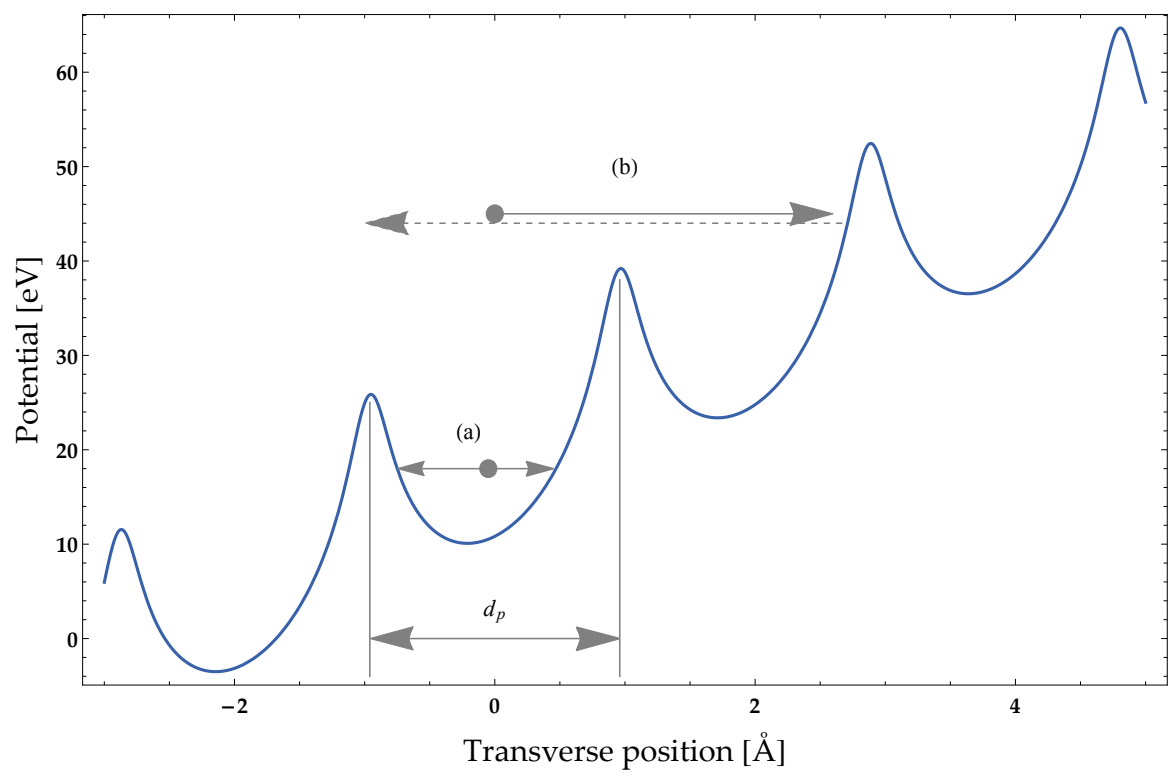

Fig. 2.9. Coherent effects conserving the transverse energy in a bent Silicon crystal. (a) Channeling process. The particle is oscillating between the planes, unable to exit. (b) An above-barrier particle traveling towards positive values of $x$ is reflected by the potential provided its incoming angle does not exceed $\theta_{b}+\theta_{c}$ (see text). It acquires a small kick in the direction opposite to channeling (i.e. towards negative $x$ ). An illustration of volume reflection is found in fig. 3.8,(b), p. 35.

\subsection{3 | Channeling mode}

The transverse energy $E_{x}$ of the incoming particle is defined by eq. (2.24). Following the classical approach, channeling requires that the transverse energy be smaller than the potential barrier :

$$
E_{x} \leq U_{0}
$$

This situation where (2.29) is fulfilled is illustrated in fig. 2.9,(a) where the particle is trapped and oscillates in one of the channels. It is evident from eq. (2.24) and eq. (2.29) that the larger the angle, the lower the probability that the particle gets channeled. For angles leading to a kinetic term of the transverse energy larger than $U_{0}$, no channeling can occur. The maximal angle allowing entry into channeling is called the critical angle and is given by :

$$
\theta_{c}=\sqrt{\frac{2 U_{0}}{p v}} .
$$

Once in channeling, a particle with transverse energy $E_{x}$ in the potential (2.25) will have a sinusoidal trajectory $x(s)$ along the channel axis where $s$ runs :

$$
x(s)=x_{m} \sin (\kappa s+\varphi)
$$




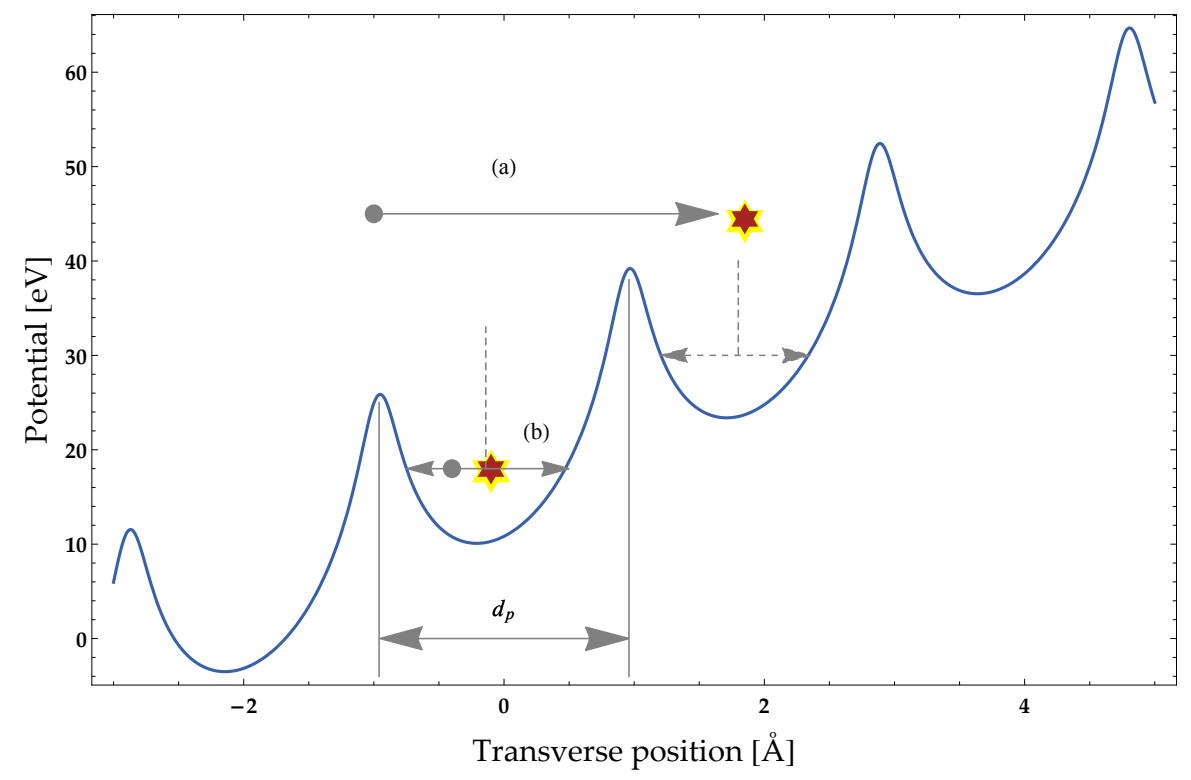

Fig. 2.10. Scattering events altering the transverse energy in a bent crystal. (a) The transverse energy of an above-barrier particle can fall below $U_{0}$ at the location of a scattering event. The particle is then captured, becoming effectively channeled. (b) The dechanneling or feed-out process is the opposite of the volume capture. A channeled particle undergoes an interaction and gains enough transverse energy to exit the channel. It then crosses the rest of the crystal in amorphous mode.

where $x_{m}$ is the amplitude of the particle transverse motion inside the channel :

$$
x_{m}=\frac{d_{p}}{2} \sqrt{\frac{E_{x}}{U_{0}}}
$$

and

$$
\kappa=\frac{2}{v d_{p}} \sqrt{\frac{2 U_{0}}{m}}
$$

The amplitude $x_{m}$ can be seen as a measure of how close the particle gets to the crystal planes throughout its oscillatory travel across the crystal. In the next chapter, it will be shown how $x_{m}$ can be related to the number of interactions undergone by the channeled particle.

\subsection{4 | Volume reflection}

As it has been shown in fig. 2.9, the potential height is shifted in bent crystals from one channel to another because of the linear centrifugal component. As a consequence, particles having a large transverse energy and moving towards the inner side of the crystal bending will encounter the potential barrier at some point of their trajectory. Assuming the crystal total bending is $\theta_{b}$, it will occur before the end of the crystal if the 
incoming angle of the particle is smaller than $\theta_{b}+\theta_{c}$ in addition to having the right sign. In that case, the particle gets actually reflected from the crystal plane, as illustrated in fig. 2.9,(b). It acquires a small kick in the direction opposite to the crystal bend.

Ref. [15] explains how to derive the value of the VR kick in a low bending case. Consider an over-barrier particle movement across planar planes. Using the harmonic approximation for the potential as well as approaching the crystal bending with a parabola, one can solve the movement equations taking into account the continuity conditions at the lattice planes (channels edges). For positively charged particles, the obtained solution is :

$$
\left|\Delta \theta_{v r}\right|=\frac{\pi}{2} \theta_{c}\left(1-2 \frac{R_{c}}{R}\right)
$$

where $R_{c}$ stands for the critical radius. Using the harmonic approximation for the potential of a straight crystal, it is given by :

$$
R_{c}=\frac{p v}{U_{h}^{\prime}\left(-d_{p} / 2\right)}
$$

Eq. 2.34 implies :

$$
R>2 R_{c}
$$

The geometrical model mentioned here is especially valid when the crystal radius of curvature is much larger than the critical one, and gives an illogical result when 2.36 is not satisfied.

\subsection{5 | Volume capture}

In a crystal like any other material, particles undergo scattering events. Coulomb scattering events on nuclei and electrons modify the trajectory of the particle. We have seen previously how, in a bent crystal, an above-barrier particle has a chance of getting reflected when the crystal planes become tangent to its trajectory. When a particle scatters near the reflection point, its transverse energy is modified, and in some cases, can fall below $U_{0}$ (see fig. 2.10(a)). The particle is then trapped in the channels until it is dechanneled or until the end of the crystal.

\subsection{6 | Dechanneling}

Channeled particles experience less interactions than particles traveling in an amorphous material. However, even this reduced amount of interactions can lead a channeled particle transverse energy to go above the barrier. In that case, the particle has enough energy to escape the channel. It is the dechanneling process, also called feed-out (see fig. 2.10(b) ). We will explain in chapter 3 how the suppression of Coulomb scattering can be evaluated. 


\subsection{7 | Illustration through experiment}

All the concepts introduced in this chapter can be displayed in one figure. It is taken from the results of the H8-RD22 experiment conducted at CERN [16] in which a strip crystal was operating in a $400 \mathrm{GeV} / \mathrm{c}$ proton beam and rotated so that the entire range of coherent phenomena could be observed. Fig. 2.11 displays beam intensity through a color scale as a function of the horizontal kick given in the crystal ${ }^{3}$ ( $x$-axis) and the crystal orientation ( $y$-axis). The distribution of particles forms 6 distinct zones. Zones 2 and 3 are obtained with the crystal in channeling orientation whereas zones 4 and 5 correspond to the VR orientation and zones 1 and 6 , to the amorphous one.

Channeling orientation means the crystal channels are perfectly aligned with the beam, optimizing the probability that particles get channeled. A particle staying channeled along the whole crystal will appear in zone 2 . If it is dechanneled, it will acquire only a fraction of the crystal nominal kick and appear in zone 3 . Rotating the crystal in the direction opposite to the bend, one suppresses channeling progressively, as particles enter now with an angle larger than $\theta_{c}$. However, their angle is small enough for them to be reflected (zone 4 - small kick in the other direction) or captured (zone 5). In the latter case, they acquire a kick corresponding to the fraction of the length of the crystal in which they were in channeling, which in turn depends on the location of the capture. As the location of the capture depends linearly on the crystal orientation with respect to the one of the beam ${ }^{4}$, we observe a linear relation between crystal orientation and deflection angle. Finally, when the crystal is further rotated, the incoming angle is not compatible anymore with any of the coherent effects that we described. The crystal acts like an amorphous device and the spread we see (zone 6) is the one due to MCS. Zone 1 is immediately reached from the channeling orientation by rotating the crystal towards the bend direction and is analog to zone 6 .

\subsection{Historical overview}

The very beginning of channeling dates back to the early twentieth century when Stark [17] postulated the anisotropic behavior of crystals. However, it is only much later that real focus was put on the subject and that several manifestations of the phenomenon were observed (for example $[18,19]$ ). In the sixties, the interest towards channeling grew rapidly and Nelson and Thompson showed in 1963 [20] that the process was a consequence of successive collisions with atoms from the lining axes or planes. At roughly the same period, the concept of continuous potential was introduced by Lehmann and

\footnotetext{
${ }^{3}$ Instead of subtracting the incoming angle from the outgoing one, the value expressed here is the outgoing angle minus the mean incoming angle from the beam.

${ }^{4}$ Only the case for small angles.
} 


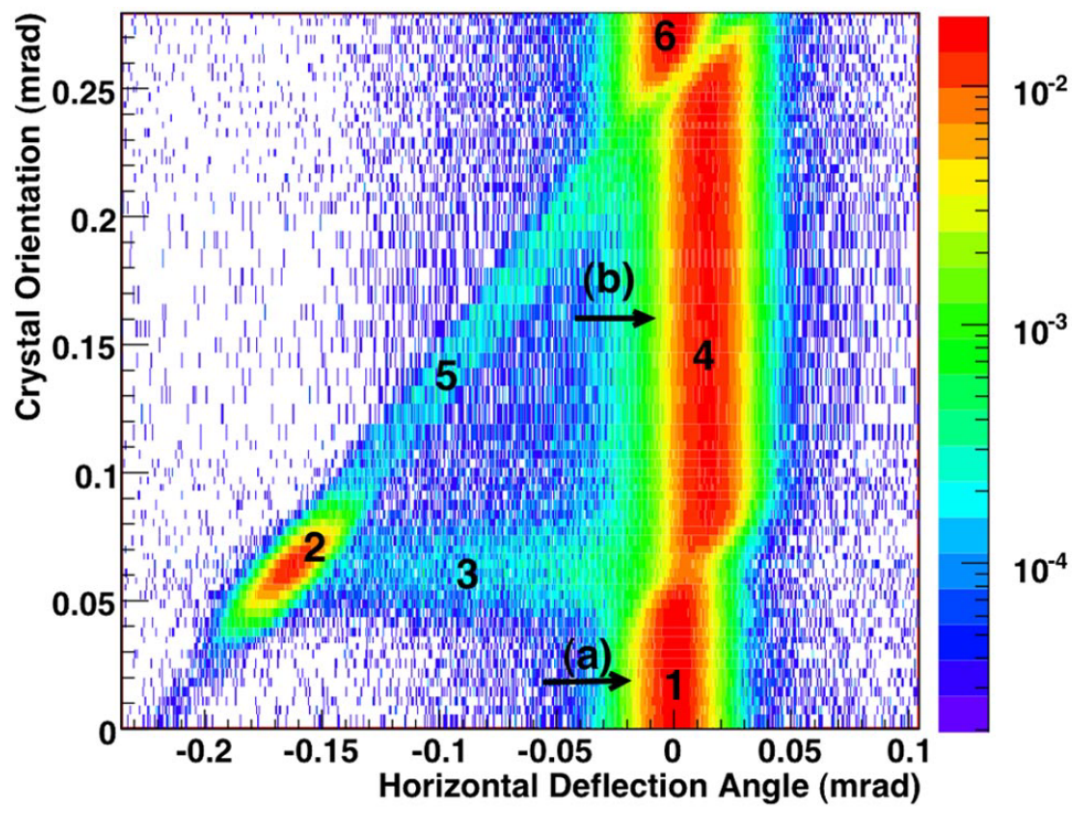

Fig. 2.11. Beam distribution (expressed in arbitrary units) at crystal exit as a function of the horizontal kick given in the crystal ( $x$-axis) and the crystal orientation ( $y$-axis). 1 and 6 : Amorphous zone, 2 : Channeling, 3 : Dechanneling, 4 : Volume reflection, 5 : Volume capture [16].

Liebfried [21], Lindhard [22] and Erginsoy [23]. This concept, along with a comprehensive view of the rest of the theory, was published in 1965 [11] in a paper that became the basis for most of the subsequent work. That paper also contains the first description of the dechanneling process, based on the diffusion theory and the notion of dechanneling length. In the following years, strong anisotropic effects were discovered for positive ions in crystals of silicon and germanium [24] and protons in tungsten [25]. In the seventies, investigations at high energies began [26] and, with them, the application of channeling to the steering of beams. That novel use of bent crystals was proposed by Tsyganov in 1976 [27] and observed few years later [28, 29]. Beam extraction through crystals was later investigated at IHEP [30], CERN [31] and Fermilab [32] where a beam extraction efficiency of 20-30\% was reached. This showed that using crystals to steer particle beams was viable. The picture of coherent effects in crystals was completed when an experiment conducted at PNPI with $1 \mathrm{GeV} / \mathrm{c}^{2}$ protons showed that those particles can also be captured between the planes [33] and finally, in 1987, when Taratin and Vorobiev predicted through simulations the volume reflection mechanism [34] which was, shortly after, confirmed experimentally.

In 1990, a beam extraction experiment was put into place at the CERN SPS [35] resulting in extraction of protons (up to $270 \mathrm{GeV} / \mathrm{c}$ ) and lead ions at $106 \mathrm{GeV} / \mathrm{c}$ per nucleon [36] and paving the way for a future crystal extraction scheme. More recently, 
crystal collimation has been under investigation in a number of accelerator complexes around the world. Beam halo collimation experiments using short bent crystals has been tested at IHEP [37], RHIC [38] and Tevatron [39]. At CERN, crystals have been considered a possible replacement of primary collimators in view of the LHC luminosity increase. In 2006 the H8-RD22 collaboration, including CERN, INFN, PNPI, JINR and IHEP began a single-pass experiment at CERN that obtained channeling efficiencies of more than $50 \%$ for crystals of different types. Strip crystals, quasi-mosaic crystals and also new types of crystals were tested. Among those, multi-strip crystals were employed for the first time. These crystals, made of several strip crystals put in succession, allow to exploit the better efficiency of VR (around 95\%) while circumventing its main limitation : the small deflection angle. Following encouraging results from the H8-RD22 experiment, the UA9 experiment has been proposed with the goal of developing a scheme of crystal collimation applicable to the LHC. In the last years, UA9 set up two experiments: one in the SPS, testing crystals behavior in a circular machine, and one on the extracted SPS beam in the North area, on the very beamline occupied few years before by H8-RD22 (more information on the latter in Chapter 4). UA9 published satisfactory results in the SPS confirming a possible use in the LHC [1, 40]. Installation of crystals in the LHC is currently under way, during the first long shutdown of the collider, as a further feasibility investigation stage. 



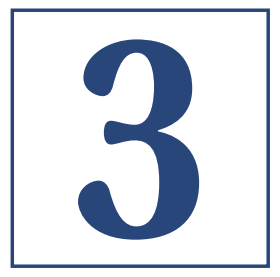

\section{THIRD CHAPTER}

\section{A Monte CARLO CHANNELING MODEL}

\subsection{Introduction}

In the previous chapter, we have introduced theoretical aspects inherent to channeling and more generally to coherent effects in crystals. In the following, methods used in an event generator designed from scratch in order to be incorporated into FLUKA [41, 42] are described. The next chapters will explain how this model can be benchmarked against results obtained by the UA9 collaboration at the H8 beamline, in CERN North experimental area.

At CERN, FLUKA is extensively used for beam-machine interaction studies. In view of the possible use of crystals as an ingredient of the future collimation system of the LHC, a comprehensive tool is needed to track particles through crystals in channeling orientation. It shall also enable the calculations of energy deposition inside of them as well as the tracking of secondaries downstream. The tools presently available in the channeling community do not allow such an investigation. On the one hand, standard tracking codes (like SIXTRACK [43] or ICOSIM [44]) have been interfaced to an empirical description of crystal effects tuned to particular conditions [5, 45]. On the other hand, more sophisticated channeling models (for example CATCH [46], or the code from A. Taratin [47]) are not yet integrated into the mentioned codes being regularly used to assist the design and the operation of big accelerators.

A comprehensive tool enabling energy deposition studies in a crystal-assisted collimation layout can be built from independently-developed codes, coupled together in order to profit from their respective strengths. Three different families of features can be identified :

- the ability to simulate particles interaction with matter 


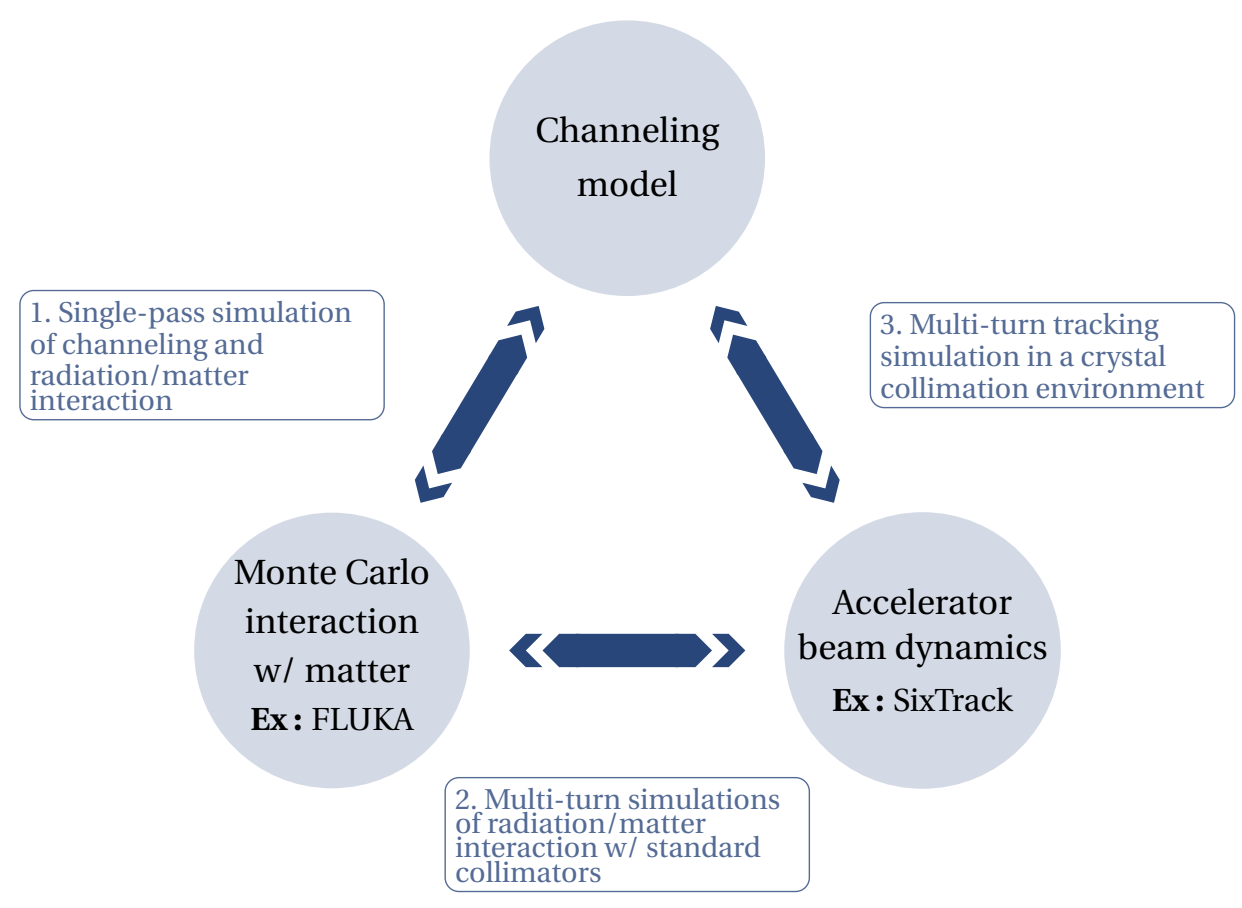

Fig. 3.1. The three components of the tool that will allow complete simulation of multiple passage of a beam in a crystal-assisted collimation layout such as the one under investigation at CERN for the LHC.

- the reproduction of coherent crystal-related phenomena

- the ability to track beam particles along the machine

A longer term goal consists in coupling these functionalities into a global tool allowing multi-turn tracking studies in a crystal-assisted collimation layout. Figure 3.1 schematically illustrates these concepts and some examples of tasks that are achievable when neighboring concepts are used in conjunction. Implementation of channeling within FLUKA (1) is the purpose of this work. Coupling between FLUKA and SixTrack (2) is currently under development at CERN [48]. Finally, work is done at CERN to embed an empirical model of channeling, dedicated to protons of $450 \mathrm{GeV} / \mathrm{c}$ and $7 \mathrm{TeV} / \mathrm{c}$ into SixTrack.

\subsection{Frames of Reference}

In this section, the frames of reference used in our model are defined and the standard orientation that will be adopted in the rest of the thesis is introduced. 


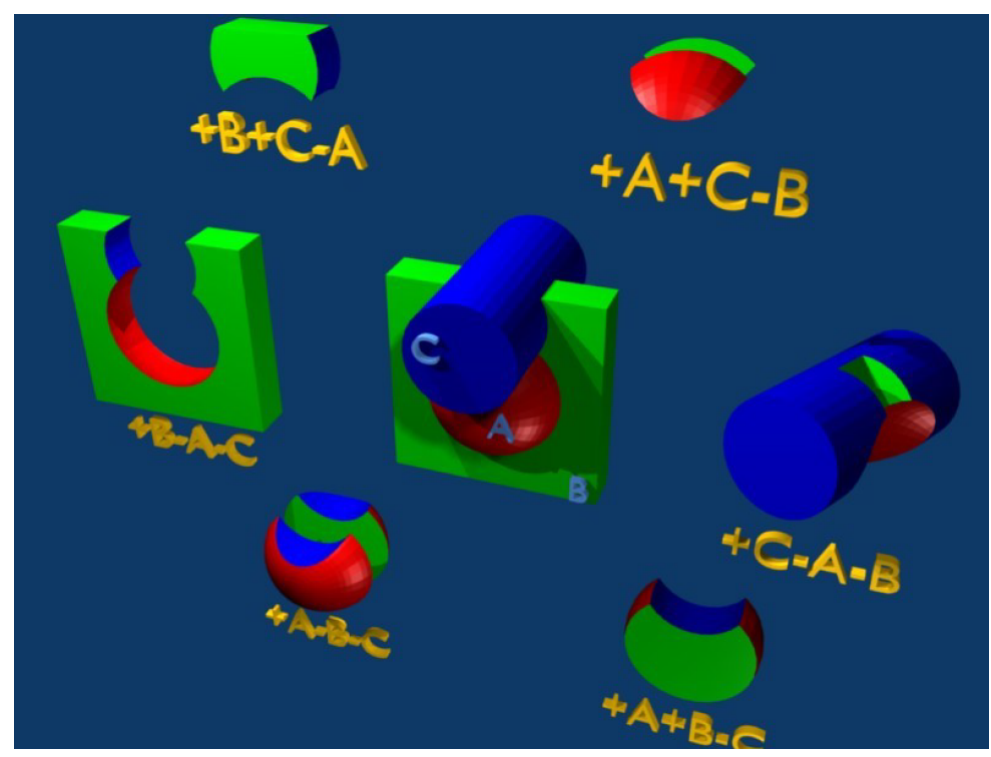

Fig. 3.2. Three bodies (in the center) and the different zones resulting from their combination. Sign "+" ("-") means that the inside (outside) of a given body is considered. A region can coincide with a single zone or with a union of zones. More information about FLUKA geometry can be found in the FLUKA Manual and the FLUKA courses material on the FLUKA website [49].

\subsection{1 | Laboratory frame of reference}

The crystal position in the laboratory and its orientation are defined through the use of the FLUKA geometry. FLUKA uses a Cartesian frame of reference and the combinatorial geometry package allowing to build complex layouts. It relies on bodies which are analytic geometric shapes such as planes, cylinders or spheres ${ }^{1}$ delimiting the space into two parts each - that can be seen as the outside and the inside of the body.

Based on the specified bodies, zones are defined through intersections of "insides" and "outsides" and regions through union of different zones. Regions are portions of space having homogeneous properties, typically representing actual components of a given element (e.g. magnet yokes, coils, vacuum chambers, collimator jaws, cooling tubes,... ). Fig. 3.2 gives examples of zones based on simple bodies. In the case of a strip crystal, the silicon region can be modeled by a cylindrical annulus sector, as shown in fig. 3.3, neglecting the saddle shape given by the holder for such a crystal (see chapter 4). FLUKA offers the possibility to apply any roto-translation or replication to the object in case of more complex geometries.

\footnotetext{
${ }^{1}$ More general quadric surfaces can also be used.
} 


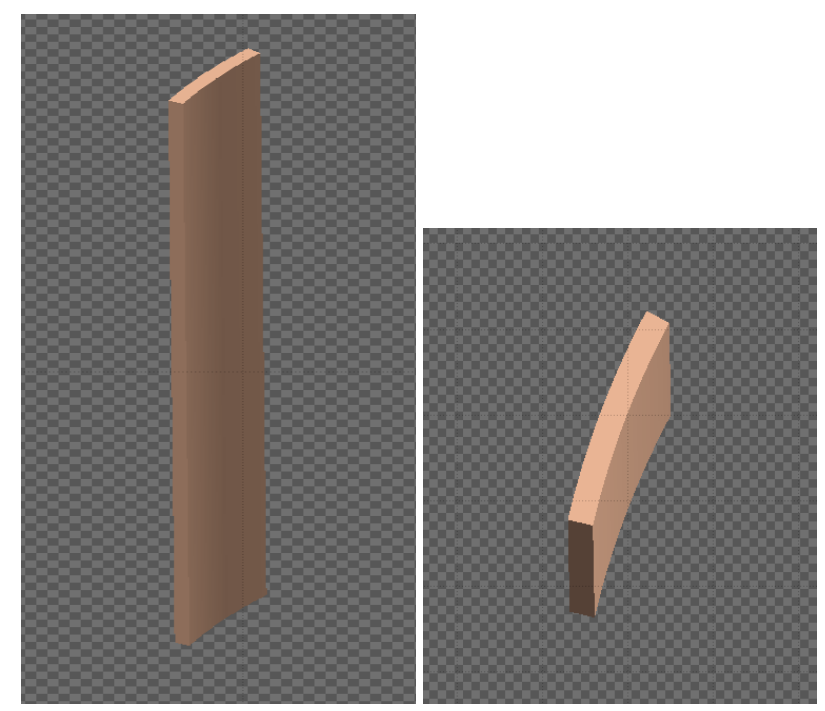

Fig. 3.3. 3D representation of a strip crystal modeled in FLUKA having an exaggerated bending angle of 15 degrees (in the LHC, bending angles are around $50 \mu \mathrm{rad}$ ); seen from the front side (left) and from the top (right).

\subsection{2 | Crystal frame of reference}

The crystal object is defined as part of the FLUKA geometry as explained above. On the other hand, the channels orientation is defined through two vectors $\boldsymbol{A}$ and $\boldsymbol{B}$ (see fig. 3.4). $\boldsymbol{A}$ gives the channel direction and, along with $\boldsymbol{B}$, defines the orientation of the crystal planes. They are parameters given independently of the crystal region characteristics coming from the FLUKA geometry. This leads to a natural reproduction of the miscut effects in crystals. It has to be emphasized that the bending is given by the user through the corresponding input card; it cannot be obtained a posteriori from the geometric shape of the crystal. If bending radius values given by the user are not consistent with the geometrical bend of the crystal region, there will be a mismatch between the channels and the crystal. For a correct orientation of the vector $\boldsymbol{A}$, perpendicular to the entry face, the channels will arrive on one of the inner lateral face if the bending value is too large, and on the outer if it is too low.

\subsection{3 | Miscut}

The miscut angle, often shortened down to miscut, is the measure of the angle between the crystal planes and the lateral end of the crystal. In absence of miscut, a particle oscillating in a channel near the crystal edge will be able to continue until the end-face. This is not the case if the channels are not parallel to the surface. Following the sign of the angle, miscut can be positive or negative. The difference between the two types of miscut is illustrated in fig. 3.5. For crystals used in synchrotrons, this difference can play 


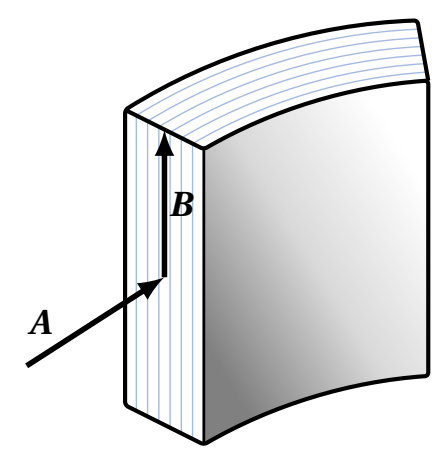

Fig. 3.4. Vectors defining the planar channels orientation. They are independent from the crystal shape defined in the FLUKA geometry. $\boldsymbol{A}$ gives the direction of the channel and $\boldsymbol{B}$ is perpendicular to $\boldsymbol{A}$ and parallel to the crystal planes.

a crucial role. In operation as part of a collimation layout, a crystal is brought inside the beam halo. Because the transverse speed of its movement is small compared to the transverse diffusion speed, the beam interacts only with the nearer part of the crystal. Positive (negative) miscut comes down to a rotation of the channels towards (opposite to) the bend. Moreover, negative miscut also induces a shortening of the channels depending on the impact parameter and the miscut angle value which is critical to the channeling properties of the device. These differences are treated extensively in the $\mathrm{PhD}$ thesis manuscript of V. Previtali [5].

In the event generator, the miscut is naturally accounted for through the fact that the channels are described independently of the crystal shape. Indeed, the user can define the crystal planes orientation and the crystal region in such a manner that the planes form a small angle with the crystal edges. The channels would then exist in the crystal up to the point where they cross the region boundary. At that location, a channeled particle would automatically lose all channeling properties per the very first condition that channeling only takes place in a CRYSTAL region.

\subsection{Torsion}

Crystals can be subject to torsion which means that they are twisted along their longest dimension (along $\boldsymbol{B}$, see fig. 3.6). Such a variation alters crystal channeling in two ways :

- The channels orientation is dependent on the $y$ coordinate. Typical values of torsion in recent experiments are of the order of several $\mu \mathrm{rad} / \mathrm{mm}$ (see chapter 5 ). For the beams dealt with in $\mathrm{H} 8$, presenting a vertical size of about $2 \mathrm{~mm}$, torsion represent a shift of about 10 to $20 \mu \mathrm{rad}$ between the bottom and the top of the beam. 


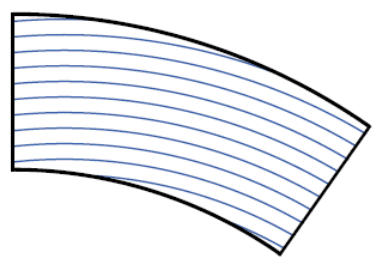

(a)

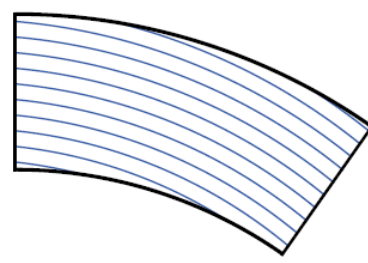

(b)

Fig. 3.5. (a) Negative miscut. In addition to changing the channels orientation, negative miscut causes channeled particles near the outer edge to exit laterally, thus partially limiting their kick. (b) The positive miscut acts in a same way for particles having very large impact parameter. In synchrotrons, this is not likely to be the case, as the vast majority of particles have a small impact parameter.

This is certainly not negligible compared to the critical angle (2.30) and leads to a decrease of the channeling efficiency as the channels cannot be ideally oriented over the whole vertical range.

- Secondly, torsion induces a modification of the radius of curvature perceived by the particle as a function of its velocity component along $y$. Indeed, if a particle $y$ coordinate at entry is different from the one at exit, the angle between the normals to the surface at the entry and exit points is different from the nominal one. However, the induced change in curvature is only noticeable for particles having a trajectory forming a large angle with the ideal channel orientation in the vertical plane (of the order of $10 \mathrm{mrad}$ ).

Torsion is modelled through a rotation of the crystal planes along the vertical axis. It takes a constant value $\mathrm{T}$, representing the rotation angle per unit of vertical length. Its effects, strongly dependent on the vertical extension of the beam, are illustrated in the case study (see section 3.7).

\subsection{Coherent behavior in a crystal}

In this section, the different stages of a particle tracking through a CRYSTAL region are explained, including conditions for channeling and volume effects and how interactions are altered in channeling regime. The main logical steps followed by the event generator are summarized in fig. 3.7. In addition, Appendix A completes the picture, giving further technical details about the implementation. 


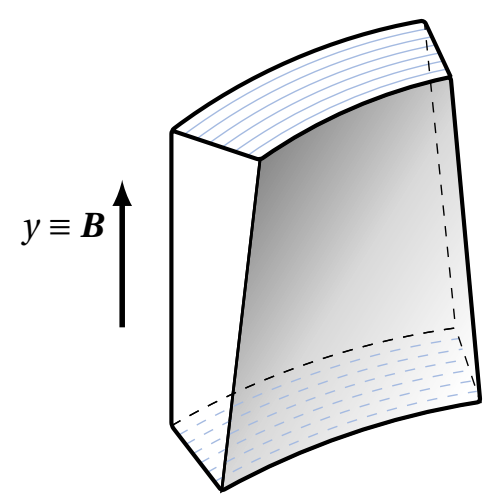

Fig. 3.6. Crystal with torsion. The orientation becomes linearly dependent on the $y$-coordinate and the effective curvature felt by the channeled particle becomes linearly dependent on the $y$-component of the particle velocity.

\subsection{1 | Entrance into the crystal region}

When a particle enters a crystal region, the first step is to calculate the angle between the particle trajectory and the crystal planes, whose orientation is defined by the user in the relevant FLUKA input card (vectors $\boldsymbol{A}$ and $\boldsymbol{B}$, see above). In case of torsion, the actual channel orientation is obtained by applying it to $\boldsymbol{A}$. The angle defines the kinetic part of the transverse energy as in (2.23) and the potential term is obtained through random sampling of the particle transverse position. A uniform probability distribution is used, accounting for the fact that impact parameters on a crystal in a collimation environment are of the orders of microns that is several orders of magnitude larger than the channel width. In consequence, the distribution of particles across the channel is considered as constant.

The transverse energy $E_{x}$, together with the sign of the angle in case of volume effects, determines what regime the particle will follow. Table 3.1 gives the conditions of entry in each of the three modes. In order to be in channeling, the incoming particle must have a transverse energy lower than $U_{0}$ which corresponds to the classical view of a particle in a potential well. $E_{x}$, calculated as per (2.24) is automatically larger than $U_{0}$ for particles incoming close to the inner side of the channel where the centrifugal term makes the potential energy exceed $U_{0}$ (see fig. 2.7). The smaller the particle incoming angle, the higher the probability it will enter channeling (the critical angle corresponding to the case where the probability drops to 0 ).

If the particle energy is lower than $U_{0}$ augmented by the kinetic energy corresponding to the bending angle of the crystal,

$$
E_{x}<U_{0}+\frac{p v}{2} \theta_{b}^{2}
$$


Table 3.1. Transverse energy thresholds at crystal entrance giving the conditions to enter modeled regimes.

\begin{tabular}{ccc}
\hline $\operatorname{sign}\left(\theta_{x}\right)$ & ENERGY CONDITION & REGIME \\
\hline- & $U_{0}+p v \theta_{b}^{2} / 2<E_{x}$ & Amorphous \\
- & $U_{0}<E_{x}<U_{0}+p v \theta_{b}^{2} / 2$ & Quasi-Channeling \\
- & $E_{x}<U_{0}$ & Channeling \\
+ & $E_{x}<U_{0}$ & Channeling \\
+ & $U_{0}<E_{x}$ & Amorphous \\
\hline
\end{tabular}

the trajectory is likely to become tangent to the crystal planes at some point, provided that it is oriented in the same direction as the crystal bend. This is true barring interactions undergone across the crystal, which can change the trajectory. Typically, in 3 $\mathrm{mm}$ of Silicon, the natural angular spread due to MCS is $\sigma=5.8 \mu \mathrm{rad}$ at $400 \mathrm{GeV}$ and $\sigma=0.37 \mu \mathrm{rad}$ at $7 \mathrm{TeV}$ (from FLUKA simulations). In order to account for this deviation which can be important for "lower" energy applications where crystals are thicker, one could increase the transverse energy limit by a factor $N_{\text {mcs }}$ proportional to $R$. Indeed, MCS is relatively more important for small $\theta_{b}$ and large $L$ and as $R=L / \theta_{b}, N_{\text {mcs }}$ scales with $R$ (not linearly though). The exact expression of $N_{\mathrm{mcs}}$ is somewhat arbitrary. Being too conservative with the choice of $N_{m c s}$ costs CPU time, as more particles will be tracked in the more time-consuming single scattering mode with the possibility of not reaching the tangency condition. Particles that do not obey $(3.1)^{2}$ are tracked in amorphous mode, which is the standard FLUKA tracking mode.

\subsubsection{Inside the channel - Oscillations}

In channeling :

1. The particle trajectory follows the channel direction. On a technical standpoint, the bent trajectories of channeled particles are modeled in FLUKA through the insertion of a virtual magnetic field, which has no other influence on the tracking. Without it, a particle trajectory is straight along a step. The presence of this field solves this issue cleanly and simply.

2. Interaction rates are lowered. Coulomb scattering which is by default applied in FLUKA in a continuous manner as MCS is applied in this case through single scattering events, whose probability is lowered as a consequence of the channeled particle staying relatively far away from the atomic lattice. Nuclear reaction events are reduced as well.

\footnotetext{
${ }^{2}$ or are oriented in the direction opposite to the crystal bend.
} 


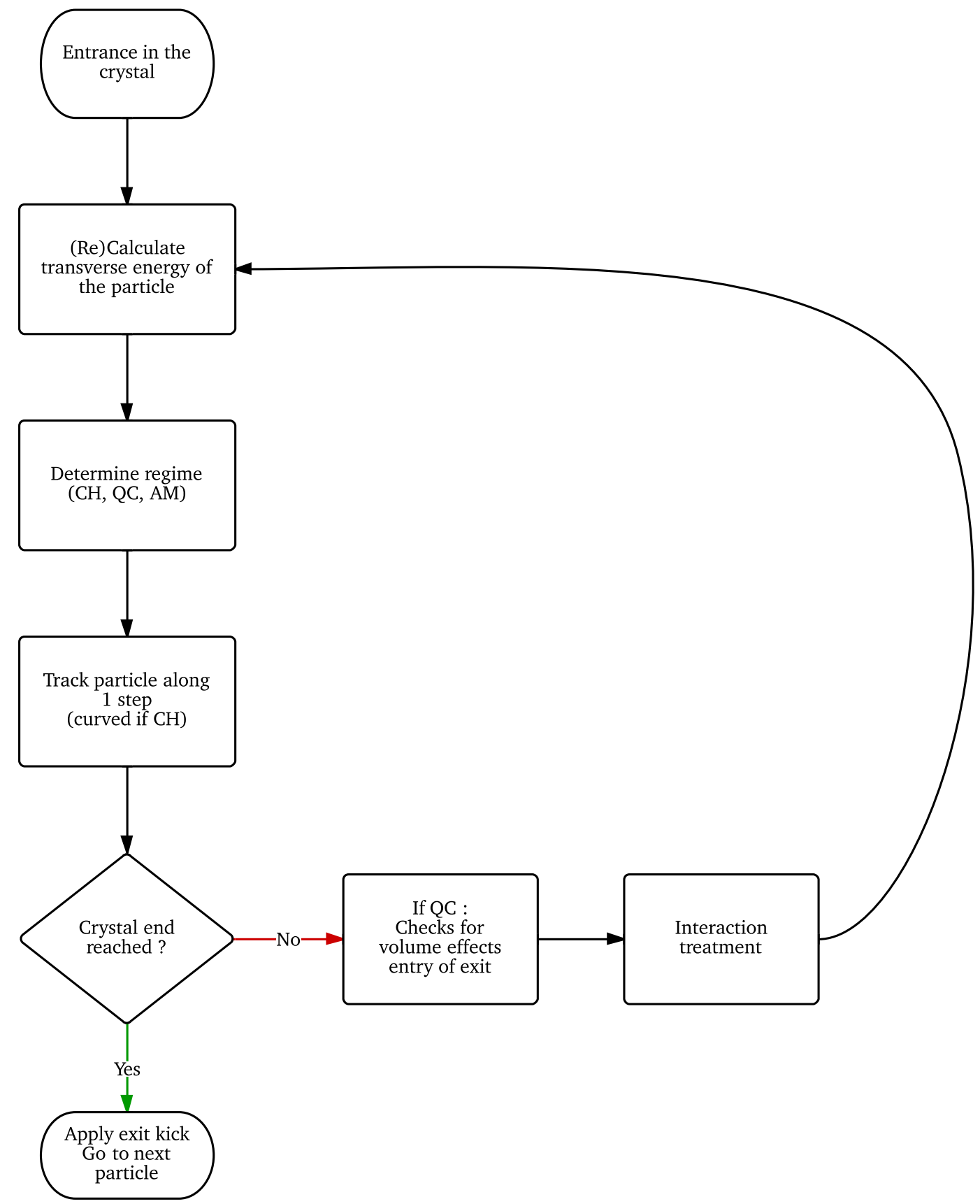

Fig. 3.7. Simplified flowchart of the main working cycle of the MC model. Upon entry in the crystal, the particle status is determined. At each step, the particle undergoes an interaction susceptible to change its status and in case of QC checks for volume effects are made. For more details about the mechanisms involved, see the text. More detailed flowcharts and their related explanations are available in appendix A. 
Channeled particles oscillate between the crystal planes. Using the harmonic approximation developed in section 2.3.2, see fig. 2.8), one can obtain equations of the particle motion in the channels, yielding sinusoidal oscillations. Transverse energy is conserved through this motion (except in case of interaction) : particles behave as if they were thrown in a gutter with their own incoming position and angle. The larger the incoming angle or the initial displacement from the channel center, the larger the amplitude of the oscillations. Those determine how close to the lattice a particle gets with some notable consequences concerning the reduction of interaction rates as explained in the following subsection.

Let us consider the harmonic potential (2.25). The equation of the particle motion is :

$$
F=m \ddot{x}=-k x
$$

the particle trajectory across the channel as a function of time is :

$$
x(t)=x_{m} \sin \left(\omega t+\varphi_{0}\right)
$$

where $\varphi_{0}$ is the phase corresponding to the initial conditions of the channeled particle and the pulsation $\omega$ is given by :

$$
\omega=\sqrt{\frac{k}{m}}=\left(\frac{2}{d_{p}}\right) \sqrt{\frac{2 U_{0}}{m}}
$$

In (3.3), $x_{m}$ represents the amplitude of the oscillations, directly obtained from the transverse energy in the harmonic approximation :

$$
x_{m}=\frac{d_{p}}{2} \sqrt{\frac{E_{x}}{U_{0}}}
$$

The oscillation amplitude is used in the determination of the Coulomb scattering form factor (see section 3.5) and the scaling factor applying to the nuclear scattering.

Assuming that $p_{x} \ll p_{z}$, the oscillation $x(z)$ is :

$$
x(z)=x_{m} \sin \left(\frac{\omega z}{v}+\varphi_{0}\right)
$$

The period is :

$$
T_{z}=\frac{2 \pi v}{\omega}=\pi d_{p} \beta \sqrt{\frac{m c^{2}}{2 U_{0}}} .
$$

where $\beta$ is $v / c$. In (110)-oriented straight silicon crystals, $T_{z}$ ranges from about 60 microns for $400 \mathrm{GeV} / \mathrm{c}$ protons or $400 \mathrm{GeV} / \mathrm{c}$ per charge lead ions to 250 microns at $7 \mathrm{TeV} / \mathrm{c}$. It is large compared to the lattice dimensions (which is consistent with the hypothesis forming the basis of channeling theory : the particle interacts with the lattice as a whole, as impact parameters from successive interactions are not independent. On the contrary, it is short compared to the crystal length (few millimeters) and the number of periods of the channeled trajectory is large. 

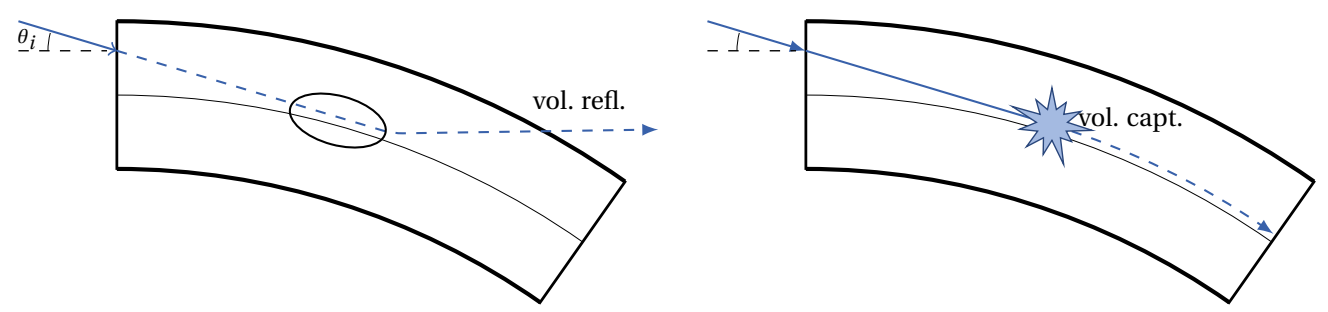

Fig. 3.8. (a) A particle entering the crystal at an angle, $\theta_{i}$, too large to become channeled. The zone of volume effects corresponding to the tangency region is circled. It ends at the tangency point with the crystal planes. (b) In this region, the particle gets captured if an interaction make its transverse energy drop below $U_{0}$. If it is not the case, the particle is reflected by the crystal planes, as shown in (a), acquiring a kick given by eq. (2.34)

\subsection{3 | Treatment of volume effects}

Quasi-channeled (QC) particles do not obey the channeling condition (2.29), however their trajectory can become tangent to the crystal planes at some point of their travel in the crystal, in general due to its curvature as shown in fig. 3.8. Close to the tangency point, these particles can undergo VC or VR (see section 2.3).

In the model under discussion, the tracking of a $\mathrm{QC}$ particle is identical to the one of an amorphous particle in single-scattering mode. Only when the angle between the crystal planes and the particle trajectory (reevaluated at each step) becomes lower than the critical angle the particle enters what is called Volume effects zone. As a consequence of interactions continuously modifying their trajectory, there is a possibility that some QC particles do not enter the zone. In that case they exit the crystal in that mode, having in effect crossed the device in single-scattering mode analogous to the amorphous mode.

The longitudinal position of the end of the zone is defined as the point where a prolonged particle trajectory would reach tangency with the crystal planes, as illustrated in fig. 3.8,(a) (where, for the sake of simplicity, it is assumed that no interactions modify the particle trajectory). The zone length corresponds to a change of the channel orientation of $\theta_{c}$ and is then :

$$
L_{v z}=R \theta_{c}
$$

Example A $450 \mathrm{GeV}$ proton beam impacts a $2 \mathrm{~mm}$-long Silicon crystal with radius of curvature $R=15 \mathrm{~m}$ along the (110) direction. The critical angle is $\theta_{c}=\sqrt{2 U_{0} / p v} \approx 10 \mu \mathrm{rad}$. The length of the tangency zone defined between the point where a straight trajectory falls within $\theta_{c}$ from the planes orientation and the tangency point is $L_{v z}=150 \mu \mathrm{m}$.

Here, unlike what is done at the entrance of the crystal where the angle of a trajectory with respect to the channel orientation is taken into account, the particle transverse kinetic energy is considered equal to zero, because of the tangency proximity. The trans- 
verse energy is then equal to the effective potential sampled at a random transverse position in $\left[-d_{p} / 2, d_{p} / 2\right]$. Fig. 3.9 shows the spectrum of energies from the effective potential sampling. As expected, an important population has a low transverse potential energy, corresponding to a position close to the channel center. As we go higher in energy and $\left|U^{\prime}(x)\right|$ increases, the population decreases until $U_{0}$ where the potential flattens again and the population reaches a maximum. Potential values above that point can only be obtained for particles close to the inner edge of the asymmetric potential. In consequence, the population of particles having $E_{x}>U_{0}$ is much reduced. The justification behind the choice $\theta_{i}=0$ is the following : a reflection takes place when a particle hits the potential barrier, as explained through fig. 2.9. At the moment of the reflection, the transverse energy is larger than the latest barrier crossed by the particle by a quantity comprised between 0 and $p v d_{p} / R$. If this quantity is exceeded, then the particle actually gets reflected by a further channel, and the same reasoning may be applied to that one.

VR occurs at the beginning of the zone if the particle transverse energy is below $U_{0}$, representing the fact that the particle cannot reach the considered channel but is reflected by the potential of a previously crossed channel, in the classical view illustrated in fig. 2.9. On the contrary, a value above $U_{0}$ means that the particle travels towards the next channel barrier and will be reflected unless it is captured (see fig. 2.10). In the model, such a particle continues its way through the crystal with its transverse energy being recalculated after every interaction. If the transverse energy drops below $U_{0}$ within a length given by (3.8), the particle is captured. If not, the particle is eventually reflected. The reflection consists of a single kick towards the outer side of the bend (see eq. (2.34). This is represented by a rotation of the particle momentum around the vector $\boldsymbol{B}$, conserving its vertical component.

In addition to the conditions on the transverse energy specified in table 3.1, particles that have $E_{x}>U_{0}$ and $\theta_{i}<\theta_{c}$ are considered to be in an intermediate state between amorphous tracking and volume reflection. As the tangency condition is fulfilled upon entry in the crystal, the deflection is applied immediately. However, the amplitude of the kick varies linearly from 0 when the particle comes in at an angle $\theta_{c}$ to $100 \%$ of the nominal VR kick for respective incoming angles $\theta_{c}$ to $-\theta_{c}$.

\subsection{4 | Crystal exit}

Upon exit from the crystal region, the channeled particle ends its oscillating movement; its final orientation, making an angle $\theta_{\text {ex }}$ with the channel direction, is given by :

$$
\theta_{\mathrm{ex}}=2 \pi \frac{x_{m}}{T_{z}} \cos \left(\varphi_{\mathrm{ex}}\right)
$$

where $\varphi_{\mathrm{ex}}$ is the phase at exit. Based on this, it is possible to calculate the exit angle of each channeled particle considering the phase as uniformly distributed in $[0,2 \pi]$ (because 


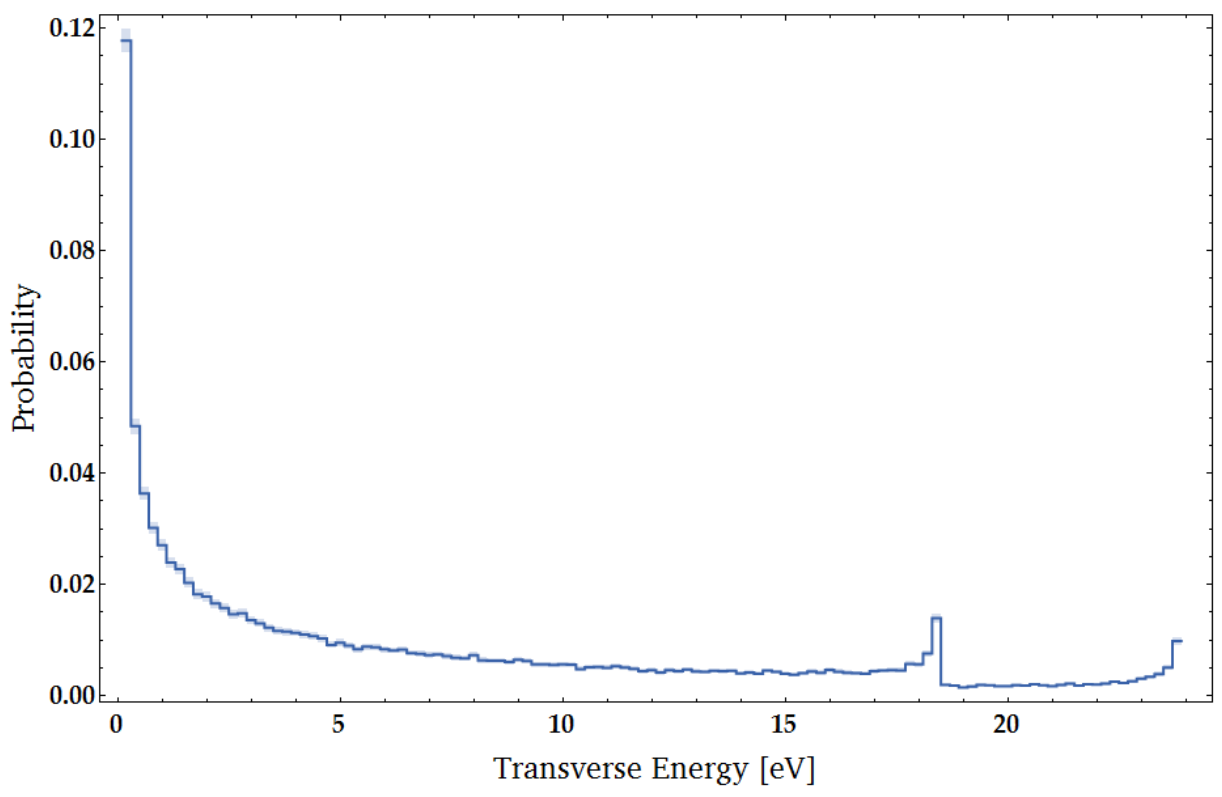

Fig. 3.9. Spectrum of transverse energies for quasi-channeled particles entering the zone of volume effects. As their kinetic energy is set to 0 , this spectrum reflects the potential part of $E_{x}$ given by the effective Molière potential (2.21). The particles with an energy smaller than $U_{0}$ are reflected. The others can be captured if a scattering event makes their energy drop below $U_{0}$ within the region of volume effects.

the period of the oscillation is much shorter than the crystal). The resulting angular distribution follows an arcsine distribution whose width is directly given by the oscillation amplitude. This method was chosen at first and yielded sharp distributions of deflection angles at crystal exit for channeled particles in disagreement with the experiment. Distribution of the horizontal deflection angles at exit are shown in fig. 3.10).

It is not surprising that the oscillations fail to naturally reproduce the exit kick distribution of channeled particles. In addition to the non-harmonicity of the potential, the use of the continuous approximation is debatable when a particle approaches the endface as the symmetry of the crystal lattice is disrupted. In absence of a more complex model taking this specificity into account, the model relies currently on a Gaussian sampling with $\theta_{c}$. As seen in fig. 3.10, it provides satisfying agreement with the experiment. The choice of the critical angle is natural and not arbitrary because by definition it represents the angular aperture of the channel. However, a possible development of the model could include an implementation of the spread coming directly from the particle motion and the crystal characteristics, consistently with the current handling of other coherent phenomena. 


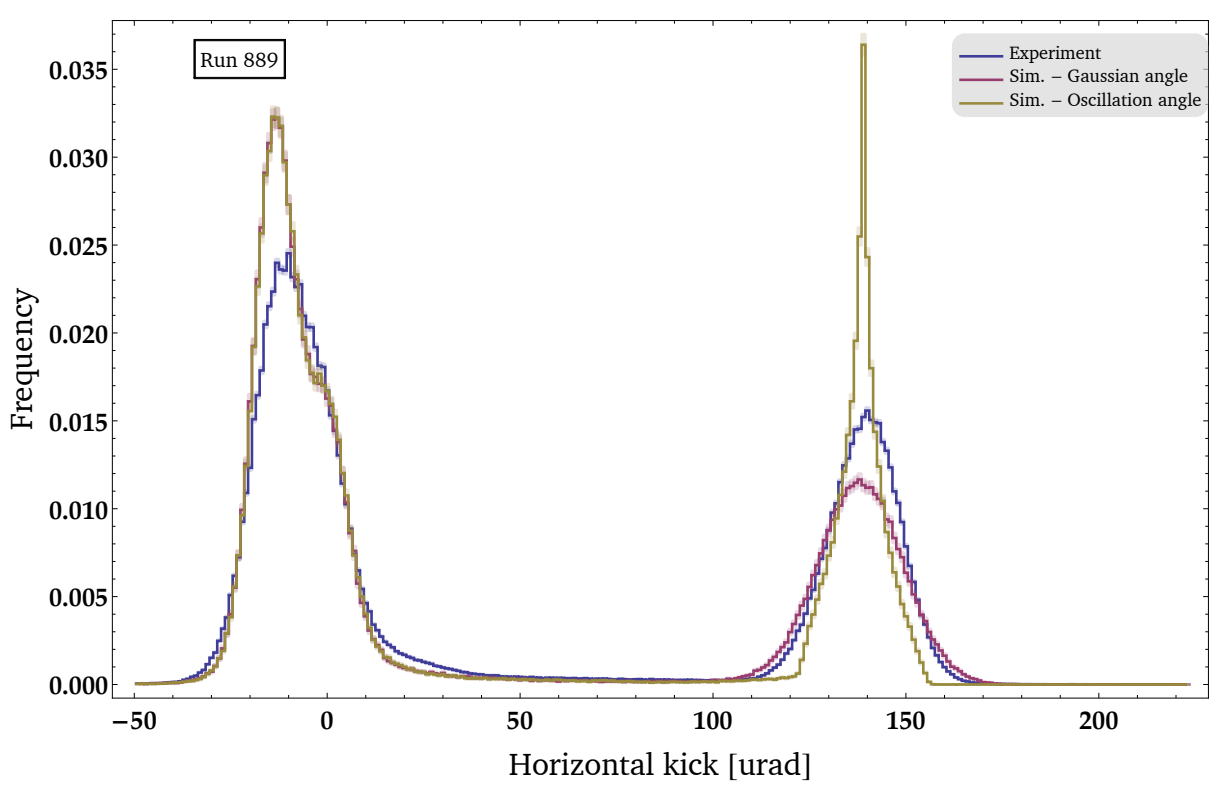

Fig. 3.10. Distribution of the horizontal deflection angle at crystal exit for run 889 (blue), a simulation where the angle if the oscillating trajectory are kept at exit (yellow), and one where the exit angle is sampled from a Gaussian distribution having $\theta_{c}$ as rms (red). The Gaussian distribution shows a good agreement with the experiment but has the disadvantage of requiring a parameter.

\subsection{Electromagnetic interactions}

Channeled particles interact less frequently with the crystal atoms than amorphous ones. As they oscillate around the center of the channel, they travel further away from the scattering centers. The smaller their transverse energy and oscillation amplitude, the further away from the lattice nodes they travel. In addition to reminding the reader about the different types of interactions reproduced in the model, this section explains how the reduction factor applied to channeled particles is calculated based on a microscopic description.

\subsection{1 | Coulomb scattering}

Charged particles passing through matter experience diffraction on the lattice nuclei or peripheral electrons EM fields. In FLUKA, Coulomb scattering is represented as a continuous phenomenon and implemented through the MCS formalism. In order for MCS to be applicable, the number of scatterings must be large ( $\gg$ 1) over the step length. At each step, a MCS deflection is applied to the particle. The amplitude of this deflection is calculated by FLUKA following the method developed in ref. [50] which explains the 


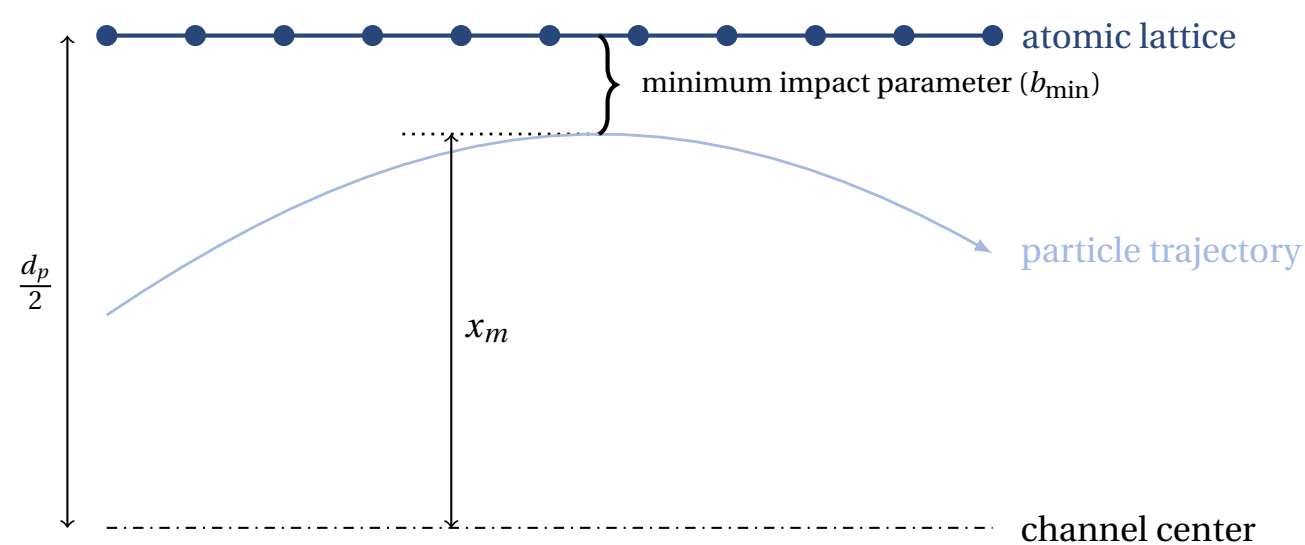

Fig. 3.11. Channeled particle oscillating around the channel center with an amplitude $x_{m}$. The resulting minimum impact parameter $b_{\min }$ with the lattice nuclei is illustrated. It is used in the calculation of the form factor reducing the number of Coulomb scatterings undergone by the particle.

application of Molière's theory with a new computation of the "wiggliness" correction ${ }^{3}$ yielding better results than previously published methods.

In FLUKA, Coulomb scattering events are computed through the formalism of MCS. The latter does not allow local corrections to the interaction rate for channeled particles, which then have to be tracked in the so-called single scattering mode where individual events are computed punctually, similarly to what is done in the tracking code for other interactions. In addition, this applies to QCH particles as well so that their entry in the zone of volume effects can be determined (it is triggered by a condition on the angle, see section 3.4.3).

Coulomb scattering on electrons and nuclei causes the direction of the particle to change. In our model, the transverse energy is reevaluated based on the new particle direction. If the transverse energy of a channeled particle becomes higher than $U_{0}$, the particle leaves its bound state and travels in amorphous mode. This process is what stands for the feed-out or dechanneling happening in real crystals. The critical point is to reproduce the interaction reduction factor as channeled particles interact less frequently. We choose to treat the problem in a semi-quantum way, by applying a form factor on the scattering event. This "filter" allows only some of the interactions to take place based on their impact parameter $b$. Fig. 3.11 sketches the idea behind the process in a classical view. The impact parameter of a particle having an oscillation amplitude $x_{m}$ obeys :

$$
x_{m}>b_{\min }=d_{p} / 2-x_{m}-\Delta x_{T} .
$$

where $\Delta x_{T}$ is the random thermal displacement of the atom. As a consequence of (3.10), we want to cut off all interactions involving impact parameters smaller than $b_{\min }$. We

\footnotetext{
${ }^{3}$ Correction introduced to take into account the differences between the straight and curved path length and between the original direction and the one after the interaction.
} 


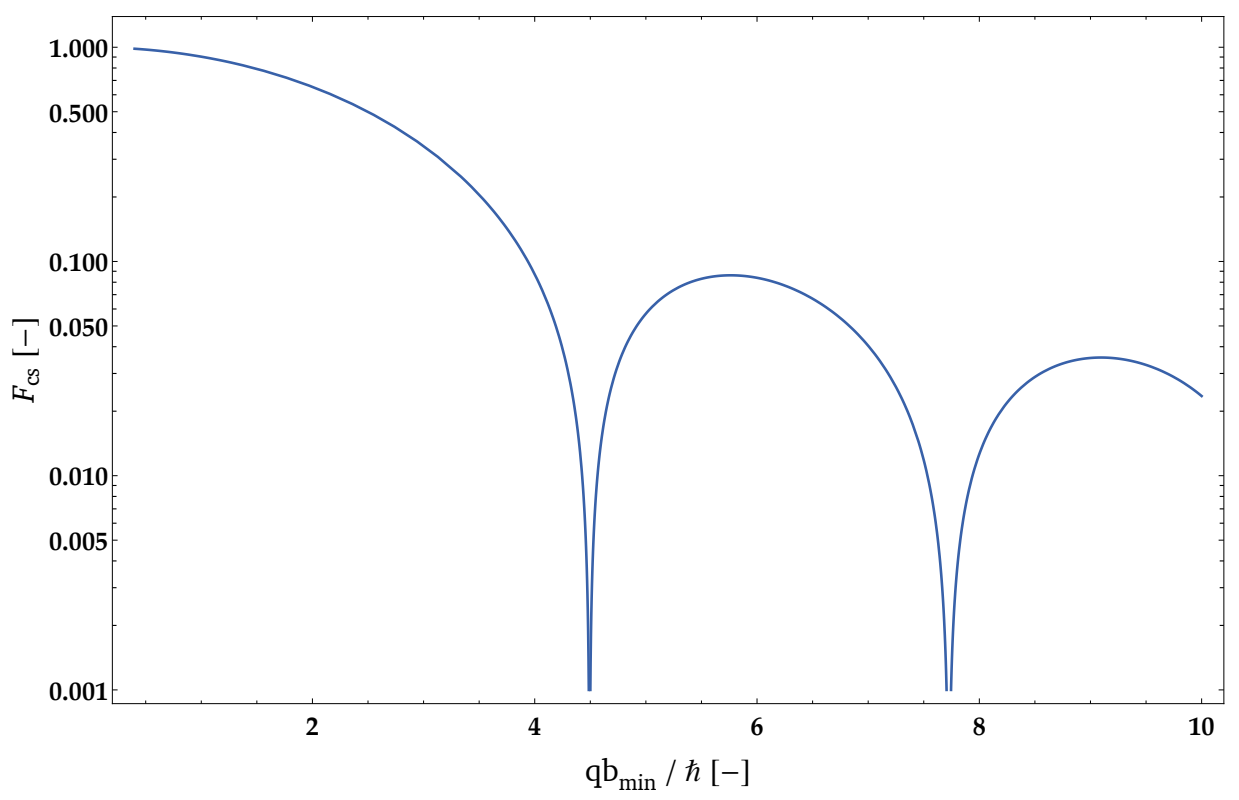

Fig. 3.12. Form factor used for the rejection of Coulomb scattering events involving a momentum transfer $q$ too large in relation with the corresponding minimum impact parameter $b_{\min }$.

are doing so through the momentum transfer $q$ of the interaction, inversely proportional to $b$. In the momentum transfer phase space, a rigid condition $b>b_{\min }$ translates to a condition on $q$ through a Fourier transform. The form factor $F_{c s}$ is obtained as the Fourier transform of a solid sphere of radius $b_{\min }$, representing a uniform distribution of the scattering centers within a distance $b_{\min }$ from the particle. It is an adaptation to Coulomb scattering of Lewin's method calculating the form factor of the nuclear interaction [51]. The radius of the nucleus becomes here the radius of the rejection sphere $b_{\min }$ :

$$
F_{c s}\left(q b_{\min }\right)=3 \frac{\left[\sin \left(\frac{q b_{\min }}{\hbar}\right)-\frac{q b_{\min }}{\hbar} \cos \left(\frac{q b_{\min }}{\hbar}\right)\right]}{\left(\frac{q b_{\min }}{\hbar}\right)^{3}}
$$

This form factor is displayed in fig. 3.12 in the case $b_{\min }=d_{p} / 4$. The effect of collisions with electrons is accounted for through the Fano correction [52], consistently with the Coulomb scattering treatment of FLUKA.

\subsection{2 | Energy Loss}

Particles traveling through matter lose energy ionizing the medium. In FLUKA, this process is computed in a continuous manner. The projectile loses a fraction of its energy at each step in a given medium. It has been shown by Appleton [53] that, considering electrons as constituents of a free gas with constant density, the energy loss of 3-11 
$\mathrm{MeV}$ protons in crystals is strongly dependent on its entrance direction. A decade later, Esbensen has shown that the energy loss of high-energy channeled particles could be formulated as [54] :

$$
\frac{\mathrm{d} E(b)}{\mathrm{d} z} \approx \frac{Z_{c}+Z(b)}{2 Z_{c}}\left(\frac{\mathrm{d} E}{\mathrm{~d} z}\right)_{\mathrm{am}}
$$

where $Z(b)$ is the average electron density on the particle trajectory for a fixed impact parameter $b$ in the transverse plane. This gives an energy loss in channeling regime which is slightly larger than one half of the one in amorphous regime.

This reduction is presently reproduced through a cut on energy of the emitted $\delta$ electrons of $1 \mathrm{keV}$, reflecting the fact that the emission of high-energy $\delta$, coming from close collisions, is reduced. This yields a factor 2.3 reduction on the energy loss in the case of a $450 \mathrm{GeV} / \mathrm{c}$-proton beam and a factor 2.7 at $7 \mathrm{TeV} / \mathrm{c}^{4}$. Although this is consistent with the expectation (3.12), a form factor similar in its principle to what has been implemented for single Coulomb scattering would reproduce the alteration of the energy loss process in crystals in a more consistent way. Specifics of these energy loss alteration in channeling are still open to debate in the channeling community.

\subsection{Nuclear interactions}

For the same reasons explained earlier in the case of scattering by the EM potential of nuclei and electrons, the amount of nuclear scattering events is reduced in channeling. This section explains how the reduction is evaluated. Nuclear interactions are separated into elastic events where the products of the reaction and the kinetic energy are preserved and inelastic events.

To reproduce the elastic and inelastic nuclear interaction rate (NIR) reduction in channeling regime, a scaling factor is applied to the FLUKA cross-section used in the crystal region for nuclear events. This scaling factor, $F_{n}$, is proportional to the density of matter the particle encounters along its trajectory. The nuclear density $P_{n}(x)$ near the crystal plane, in units of the density in amorphous regime, is described with a Gaussian distribution [55] shown in fig. 3.13 :

$$
P_{n}(x)=\frac{d_{p}}{\sqrt{2 \pi u_{t}^{2}}} \exp \left(-\frac{\left(d_{p} / 2-x\right)^{2}}{2 u_{t}^{2}}\right),
$$

where $u_{t}$ is the mean thermal displacement of silicon atoms already introduced in chapter $2(0.075 \AA$ at room temperature). Considering the oscillating particle trajectory, one

\footnotetext{
${ }^{4}$ From FLUKA.
} 


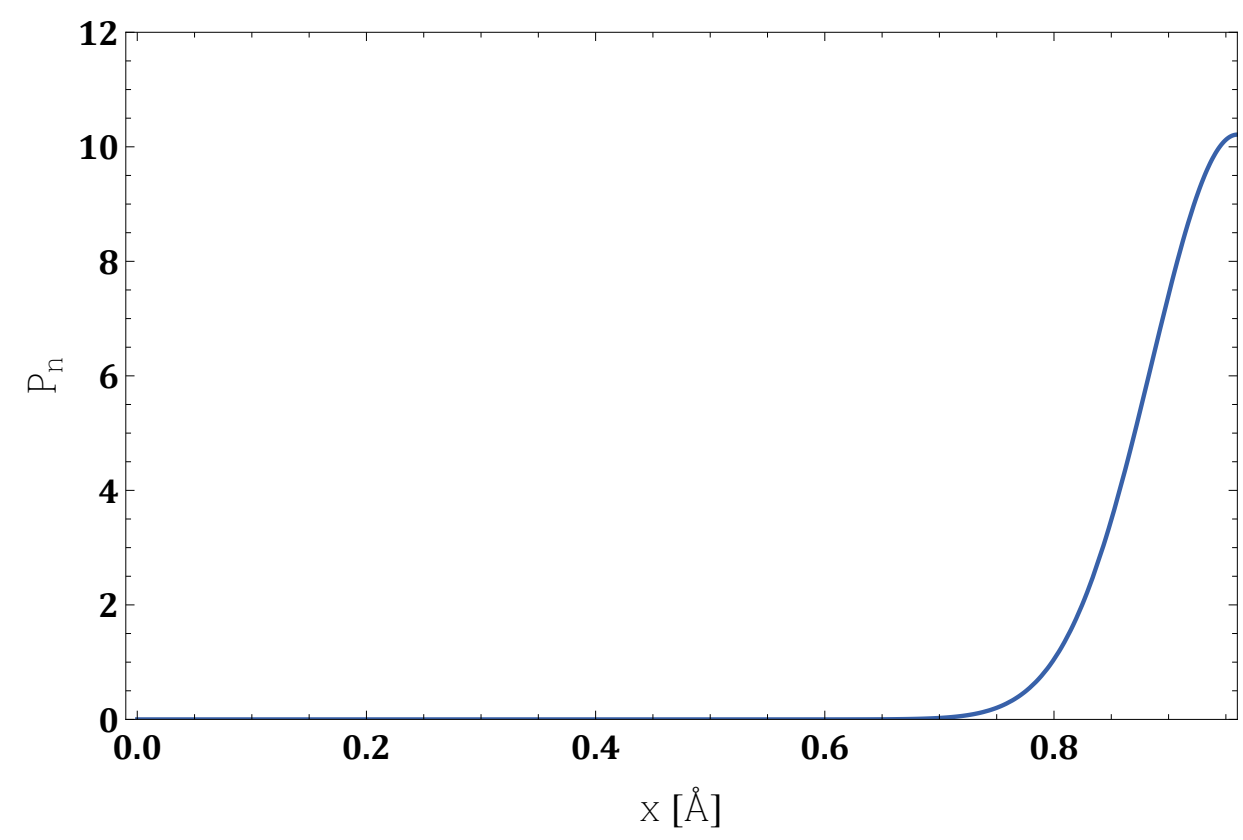

Fig. 3.13. Nuclear density in a $\mathrm{Si}<110>$-half channel given by eq. (3.13) in units of the one in amorphous regime. Values exceed 1 towards the channel edge at $d_{p} / 2$.

obtains for $F_{n}$ :

$$
F_{n}=\frac{d_{p}}{\sqrt{2 \pi u_{t}^{2}}} \frac{1}{\pi} \int_{0}^{\pi} \exp \left(-\frac{\left(d_{p} / 2-x_{m} \sin (\varphi)\right)^{2}}{2 u_{t}^{2}}\right) \mathrm{d} \varphi
$$

As displayed in fig. 3.14, $F_{n}$ stays very low for amplitudes up to $80 \%$ of the channel halfwidth. On the other hand, large-amplitude oscillations lead to an increase of the close collision probability. In fact, the effective increase in the nuclear interaction length for all channeled particles can be seen as the folding of $F_{n}$ with the distribution of the channeled particles oscillation amplitudes. Beyond $d_{p} / 2$, the scaling factor keeps increasing with the amplitude. This can be understood by picturing a sine trajectory crossing the lattice. If its amplitude is only slightly larger than $d_{p} / 2$, the part of the path near the lattice will be maximized and the crossed density as well. By definition, particles having such trajectories are not channeled. Quasi-channeled particles, however, can show this type of behavior and follow pseudo-oscillations in the crystal lattice in spite of their travel through the planes (their transverse energy is above $U_{0}$ ). Their interaction rate can even be higher than in amorphous regime but since their oscillation amplitude is not defined, we do not apply to them any scaling form factor.

Table 3.2 gives NIR for different types of beams impacting a $2 \mathrm{~mm}$-long strip crystal in channeling orientation :

1. $400 \mathrm{GeV} / \mathrm{c}$ protons 


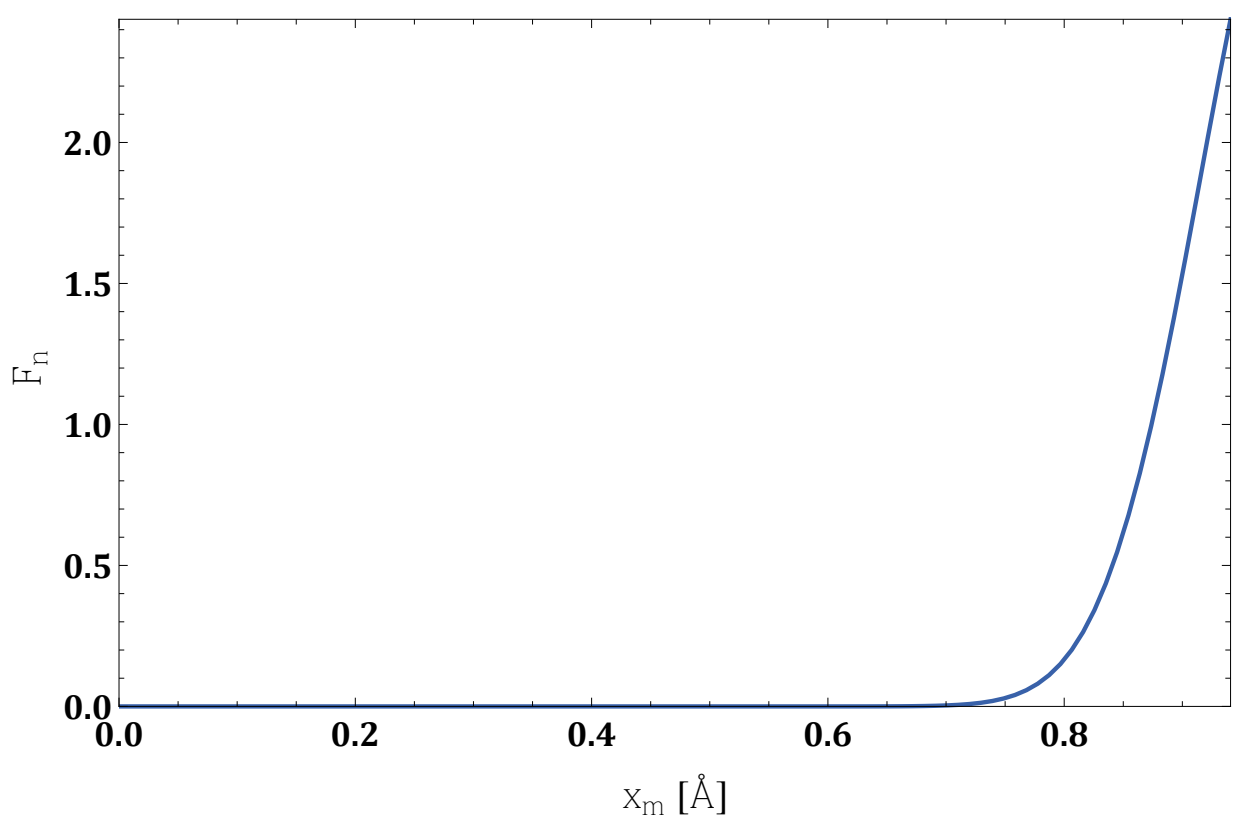

Fig. 3.14. Nuclear cross-section multiplying factor as a function of the oscillation amplitude of the channeled particle. Under this model, the maximum is reached for oscillation amplitudes larger than the channel width, maximizing the path near the atomic lattice.

\section{2. $7 \mathrm{TeV} / \mathrm{c}$ protons}

3. $400 * 82 \mathrm{GeV} / \mathrm{c}$ lead ions $(157.7 \mathrm{GeV} / \mathrm{c} / \mathrm{u})$

When interacting with a crystal in channeling orientation, not all particles will be channeled. The percentage of particles being trapped is strongly dependent on crystal parameters (torsion, bending) and on the beam divergence. Therefore, for two same crystals in channeling orientation in different beams, the measured overall channeling efficiency can be very different. This is the reason why a careful estimation of the crystal performance must rely on narrow filters reducing populations of incoming beam particles down to ensembles having similar properties. As a consequence, in order to evaluate the reduction of the NIR, one should calculate it :

- for channeled particles only and

- for particles traveling through the crystal in amorphous regime only,

always for a crystal in channeling orientation. The ratio between one and the other gives the reduction factor, which is independent from the channeling efficiency. On the other hand, if one wants to compute the global NIR in a crystal put in channeling orientation inside a beam, the channeling efficiency is critical. 
Table 3.2. Suppression of nuclear interaction for different proton beams incoming on a $2 \mathrm{~mm}$-long crystal in channeling orientation : the particle momentum, the beam divergence and the crystal bending are given in the left part of the table. Column $4(5), \%_{n, a m}\left(\%_{n, c h}\right)$ gives the percentage of particles in amorphous (channeling) regime undergoing a nuclear reaction in the crystal. Dechanneled and captured particles are distributed among those two groups according to the corresponding event location. Column 6 lists the reduction factor $\%_{n, a m} / \%_{n, c h}$. In the right part of the table, "\% chann." gives the fraction of particles impacting the crystal having been channeled (corrected for dechanneling and VC) and the last column gives the global NIR.

\begin{tabular}{ccc|ccc|cc}
\hline $\begin{array}{c}\mathrm{p} \\
{[\mathrm{GeV} / \mathrm{c}]}\end{array}$ & $\begin{array}{c}\theta_{b} \\
{[\mu \mathrm{rad}]}\end{array}$ & $\begin{array}{c}\sigma_{x^{\prime}} \\
{[\mu \mathrm{rad}]}\end{array}$ & $\%_{n, a m}$ & $\%_{n, c h}$ & $\begin{array}{c}\text { REDUCTION } \\
\text { FACTOR }\end{array}$ & $\%_{\text {chann. }}$ & $\%_{n, \text { tot }}$ \\
\hline 400 & 0 & 10 & $0.667(6)$ & $0.0528(11)$ & $12.6(8)$ & $53.0(1)$ & $0.342(5)$ \\
400 & 150 & 10 & $0.663(9)$ & $0.0523(39)$ & $12.8(9)$ & $47.6(1)$ & $0.371(4)$ \\
400 & 150 & 0 & $0.655(12)$ & $0.0246(17)$ & $25.4(1.7)$ & $85.7(1)$ & $0.115(7)$ \\
7000 & 0 & 2.4 & $0.674(6)$ & $0.138(5)$ & $4.88(19)$ & $53.9(1)$ & $0.385(5)$ \\
7000 & 50 & 2.4 & $0.661(5)$ & $0.135(6)$ & $4.90(22)$ & $20.6(1)$ & $0.553(5)$ \\
7000 & 50 & 0 & $0.663(6)$ & $0.0787(27)$ & $8.43(30)$ & $54.8(1)$ & $0.343(5)$ \\
\hline
\end{tabular}

The NIR in channeling is obtained by taking the number of nuclear events divided by the effective number of channeled particles having crossed the crystal. Here, effective means that dechanneled and captured particles are counted as belonging to both groups with a weight corresponding to their path fraction in the respective mode. For example, let us consider a particle having been trapped in a channel, then being dechanneled at some point $z_{d}$ in the crystal. If it undergoes a nuclear collision while traveling through the remainder of the crystal region in amorphous mode, the event is recorded as an "amorphous" event, but the information about the fact that no interaction occurred in the channeled part of the path would be lost. To remedy that, the particle is counted towards $\left(L_{z}-z_{d}\right) / L_{z}$ in amorphous regime and $z_{d} / L_{z}$ in channeling, where $L_{z}$ is the crystal length. A similar reasoning can be held with particles that are (also) captured.

Table 3.2 shows results of the model for the NIR in channeling and amorphous regime and the related reduction factor. Values in parentheses indicate a confidence level of $95 \%{ }^{5}$. The nuclear rate for amorphous-mode particles $\%_{n, \text { am }}$ reflects the cross-sections values used in FLUKA. They do not vary strongly for protons between $400 \mathrm{GeV}$ and $7 \mathrm{TeV}$, which explains the similarity between the rates; observed variations are inside the statistical uncertainty. For channeled particles (only), the rate $\%_{n, c h}$ depends additionally on the distribution of oscillation amplitudes. The bigger the amount of particles confined near the channel center, the smaller the scaling factor. The distribution of amplitudes in the channel is represented in fig. 3.15. For a straight crystal (first row in table 3.2), the potential barrier is a little bit higher $-21 \mathrm{eV}$ instead of $18 \mathrm{eV}$ as illustrated in fig. 3.16 - but the effect on the distribution of oscillation amplitudes is small and the reduction

\footnotetext{
${ }^{5}$ Details about confidence intervals determination are available in appendix B.
} 


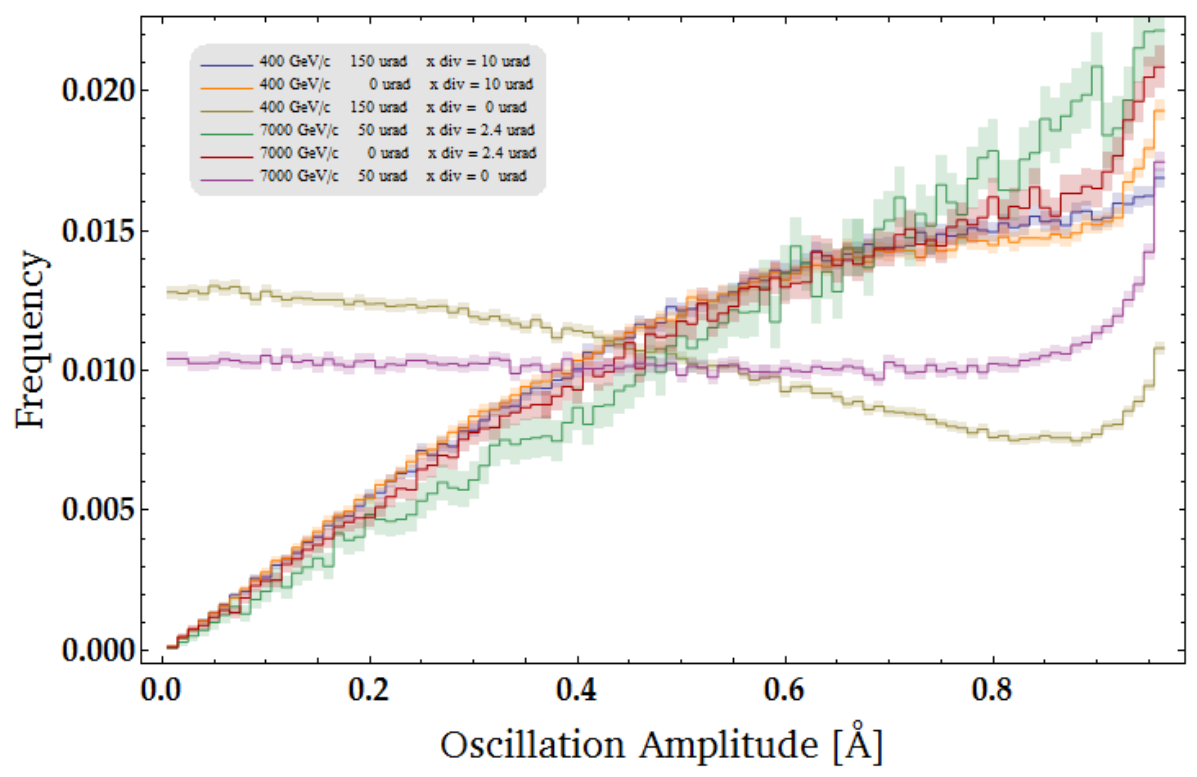

Fig. 3.15. Distribution of protons oscillation amplitudes in channeling for the 6 cases from table 3.2. In absence of divergence, the distribution results solely from the uniform sampling of the lateral position in the effective potential. The divergence considered in the other cases is taken as close to the critical angle at the corresponding energy. For them, small amplitudes are much rarer for they need both a small incoming angle and a favorable position in the channel.

factor stays of the same order. The effect is much clearer when the beam divergence is zero (third row). Without kinetic component, the average transverse energy is reduced substantially, leading to a much smaller average amplitude.

The reduction factor is high when particles in channeling have small oscillation amplitudes. On the contrary, it is low when the distribution of transverse energies is wide and flat (typically when the channeling rate is low). For example, at $7 \mathrm{TeV}$, for a divergence close to the critical angle (about $2.4 \mu \mathrm{rad}$ ), the potential barrier is low at $9.1 \mathrm{eV}$. This means that transverse energy values can strongly exceed the potential barrier and for particles having $E_{x}<U_{0}$, the distribution is extremely flat and the reduction rate is lower.

For lead ions, nuclear rates are much higher than for protons (larger cross-sections) but the scaling factors are calculated in the same way, and lead to similar reduction factor values assuming the same oscillation amplitude distributions. Values given in the last row of table 3.2 are due to inelastic events only as nuclear elastic interactions for heavy ions are not implemented yet into FLUKA. According to [56], the intrinsic cross section of those elastic events should be close to the inelastic one (considering no EM interaction). The problem lies in the interferences existing between Coulomb and nuclear scattering. Taking into account elastic interactions would lead to double-counting. For this reason, 


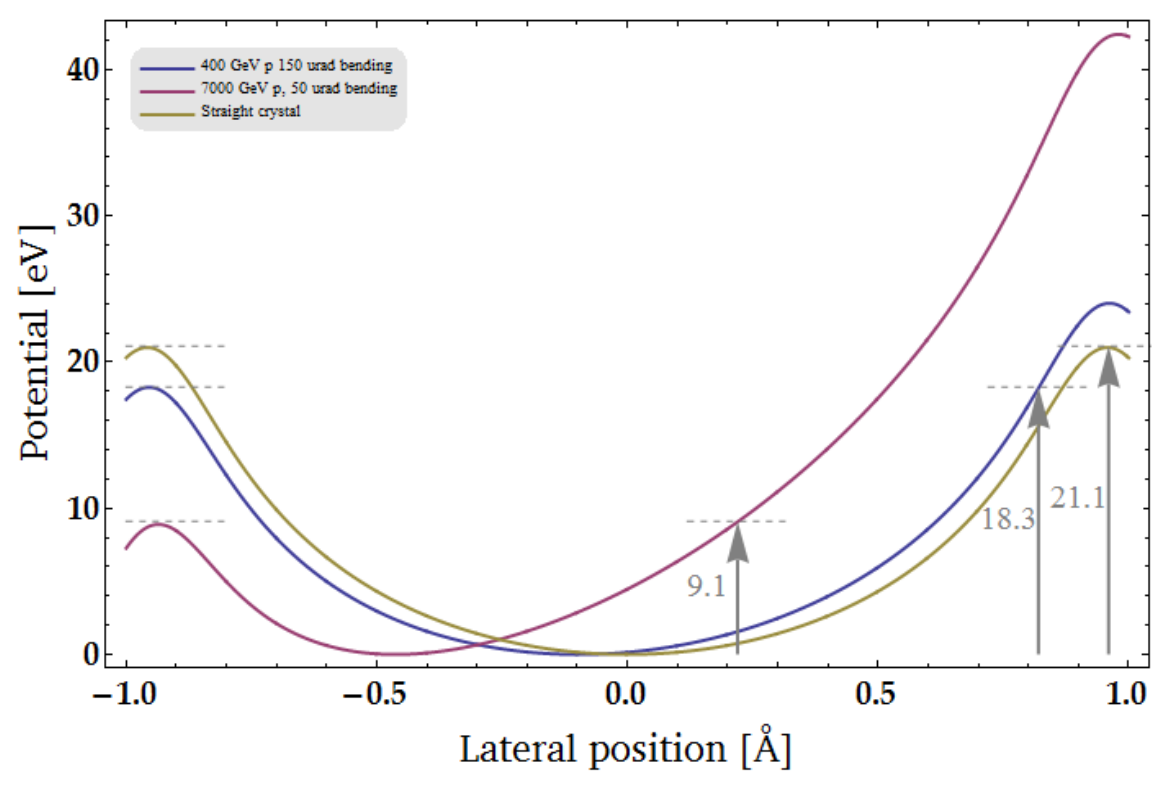

Fig. 3.16. Moliere potential profile across a channel for the proton cases from table 3.2. The centrifugal component, dependent on the $p v$ product largely reduces the depth of the potential well in the case of $7 \mathrm{TeV}$ protons. Depths are respectively of $21.1 \mathrm{eV}, 18.3 \mathrm{eV}$ and $9.1 \mathrm{eV}$ for protons in a straight crystal, for $400 \mathrm{GeV} / \mathrm{c}$ protons in a bent crystal (bending $150 \mu \mathrm{rad}$ ) and for $7 \mathrm{TeV} / \mathrm{c}$ protons in a bent crystal (bending $50 \mu \mathrm{rad}$ ).

NE events in heavy ion-hadron collisions are not implemented in FLUKA and the question of their incorporation is still open.

\subsection{Case study}

In this section, the model results are shown for several sets of input parameters, reflecting alterations of a base scenario, each of them changing one of the main parameters of the simulation. Table 3.3 summarizes the input parameters for the different cases treated in this section.

\subsection{1 | Case 0 : Base conditions}

Let us consider a $400 \mathrm{GeV} / \mathrm{c}$ proton beam incoming along the $<110>$ direction on a Silicon crystal with dimensions $2 \times 10 \times 2 \mathrm{~mm}^{3}$ along $x, y$ and $z$ respectively. The crystal is oriented as in fig. 3.4, p. 29 with $\boldsymbol{A}$ along the $z$ axis. The crystal is bent with a radius of curvature of $10 \mathrm{~m}$ which means a bending angle of $200 \mu \mathrm{rad}$. The beam has no divergence 
Table 3.3. Variable input parameters of the case study. Other parameters are the crystal transverse area $\left(2 \times 10 \mathrm{~mm}^{2}\right)$, the beam spread along the vertical axis $\left(\sigma_{y}=1 \mathrm{~mm}\right)$ and the vertical divergence : $\sigma_{y^{\prime}}=10 \mu \mathrm{rad}$ (which matters only in case of torsion). The blue color emphasizes parameters differing from the base scenario.

\begin{tabular}{llcccccc}
\hline CASE & & 0 & 1 & 2 & 3 & 4 & 5 \\
\hline Particle type & & $\mathrm{p}^{+}$ & $\mathrm{p}^{+}$ & $\mathrm{p}^{+}$ & $\mathrm{p}^{+}$ & $\mathrm{p}^{+}$ & ${ }^{208} \mathrm{~Pb}^{82+}$ \\
Momentum & {$[\mathrm{GeV} / \mathrm{c} / \mathrm{u}]$} & 400 & 400 & 400 & 400 & 7000 & 158 \\
Radius & {$[\mathrm{m}]$} & 10 & 10 & 10 & 2 & 40 & 20 \\
Bending Angle & {$[\mu \mathrm{rad}]$} & 200 & 200 & 200 & 200 & 50 & 100 \\
Crystal Length & {$[\mathrm{mm}]$} & 2 & 2 & 2 & 0.4 & 2 & 2 \\
Hor. Div. $\sigma_{x^{\prime}}$ & {$[\mu \mathrm{rad}]$} & 0 & 10 & 0 & 5 & 5 & 5 \\
Torsion & {$\left[\mu \mathrm{rad} . \mathrm{mm}^{-1}\right]$} & 0 & 0 & 10 & 2 & 2 & 2 \\
\hline
\end{tabular}

in $x$ and $y$ and the vertical spread is $\sigma_{y}=1 \mathrm{~mm}$. The spread in $x$ is not relevant because all the particles are assumed to hit the crystal.

Fig. 3.17 displays the output of the base scenario. The graph shows the same information as the one ending chapter 2 : the beam distribution is displayed as a function of the crystal orientation and the particles horizontal kick gained in the crystal. The use of the crystal orientation is here equivalent to the use of the particle incoming angle, as the divergence is zero. In other situations where it is not the case anymore, there would be a vertical stretching (on the graph) of the particle distribution inherent to the representation of a divergent beam through the value of the crystal orientation which can be viewed, in an equivalent frame of reference where rather the orientation of the beam is changing, as the mean incoming angle of the beam.

The transition out of the channeling orientation is very sharp in this case. Only particles with $\theta_{x}$ in $\left[-\theta_{c}, \theta_{c}\right]$ can be channeled. The channeled particles with a relatively large incoming angle have a larger probability of being dechanneled, as shown by the higher density on the edges of the channeling orientation zone. This is because those particles start with a transverse energy closer to $U_{0}$, making them more likely to be dechanneled. In addition, they oscillate closer to the lattice and their interaction rate is not lowered as much as for particles having a very small incoming angle and oscillating near the channel center (see section 3.5).

In the VR orientation, captured particles are clearly seen. They acquire a kick depending on the location of their capture (assuming they stay in channeling for the rest of the crystal length). Particles that are captured near the entry-face are found close to the channeling spot, whereas particles captured at the very end are close to the junction between VR and AM. That junction appears tilted in the graph. This is due to the dependence of VR occurrence on MCS in the former part of the crystal. In other words, 
a particle being hardly quasi-channeled might be deflected via MCS by an angle large enough that it prevents the particle from reaching tangency and thus from having the possibility of being reflected or captured. The opposite is also true for particles with $E_{x}$ barely over-threshold for QC : when applying the QC condition upon entry in the crystal, a transverse energy margin corresponding to the critical angle is taken into account in order to allow single scattering tracking for particles that would not reach tangency unless they are deflected by MCS towards the bend. As a consequence, for incidence angles at the far-end limit between VR and AM, there is a dependence between the kick given to the particle by MCS and the occurrence or not of the reflection process, hence the transition appears tilted.

Even at small angles, not all particles get channeled, as per the condition on the transverse energy which can be violated due to the entrance position in the channel. A sharp condition on the incoming angle is separating these particles between AM if the angle is negative and QC if the angle is positive. This creates the abrupt change in regime seen in the figure which is going to be remedied in the closing stages of the work. Solutions to remove this artifact are discussed in chapter 5 .

The attentive reader will remember figure 2.11, which gives similar information for a run out of the H8-RD22 experiment. In that case, it must be pointed out that the value recorded on the $x$ axis is the deflection angle $\Delta_{e} x^{\prime}$ measured as

$$
\Delta_{e} x^{\prime}=x_{\text {out }}^{\prime}-\bar{x}_{\text {in }}
$$

where $\bar{x}_{\text {in }}^{\prime}$ is the mean incoming angle which is equivalent to the crystal orientation. The case study presented in the following gives rather the real deflection angle $x_{\text {out }}^{\prime}-x_{\text {in }}^{\prime}$, value of direct interest in the context of collimation. The tilt seen in fig. 2.11 is due to the fact the deflection angle is taken as the difference between the outgoing angle and the mean incoming angle. If we decompose the outgoing angle as the addition of the incoming angle and the deflection given by the crystal $x_{\mathrm{cr}}^{\prime}$, eq. (3.15) becomes :

$$
\Delta_{e} x^{\prime}=x_{\mathrm{in}}^{\prime}+x_{\mathrm{cr}}^{\prime}-\bar{x}_{\mathrm{in}}
$$

Here, $x_{\text {in }}^{\prime}-\bar{x}_{\text {in }}$ is the incoming angle of the individual particle in the crystal frame of reference. It is therefore natural from eq. (3.16) that there be a linear dependence between the deflection of channeled particles and their individual incoming angle.

This is not the case if one scores the deflection as purely the difference between the particle outgoing and incoming angle, and that is what is illustrated in the following figures.

In addition to these qualitative observations, the reader can find in figs. 3.17 to 3.22 joint tables the following quantities ${ }^{6}$ :

\footnotetext{
${ }^{6}$ The respective values are not given directly by the simulation but are rather resulting from a data analysis done $a$ posteriori on the simulation output.
} 


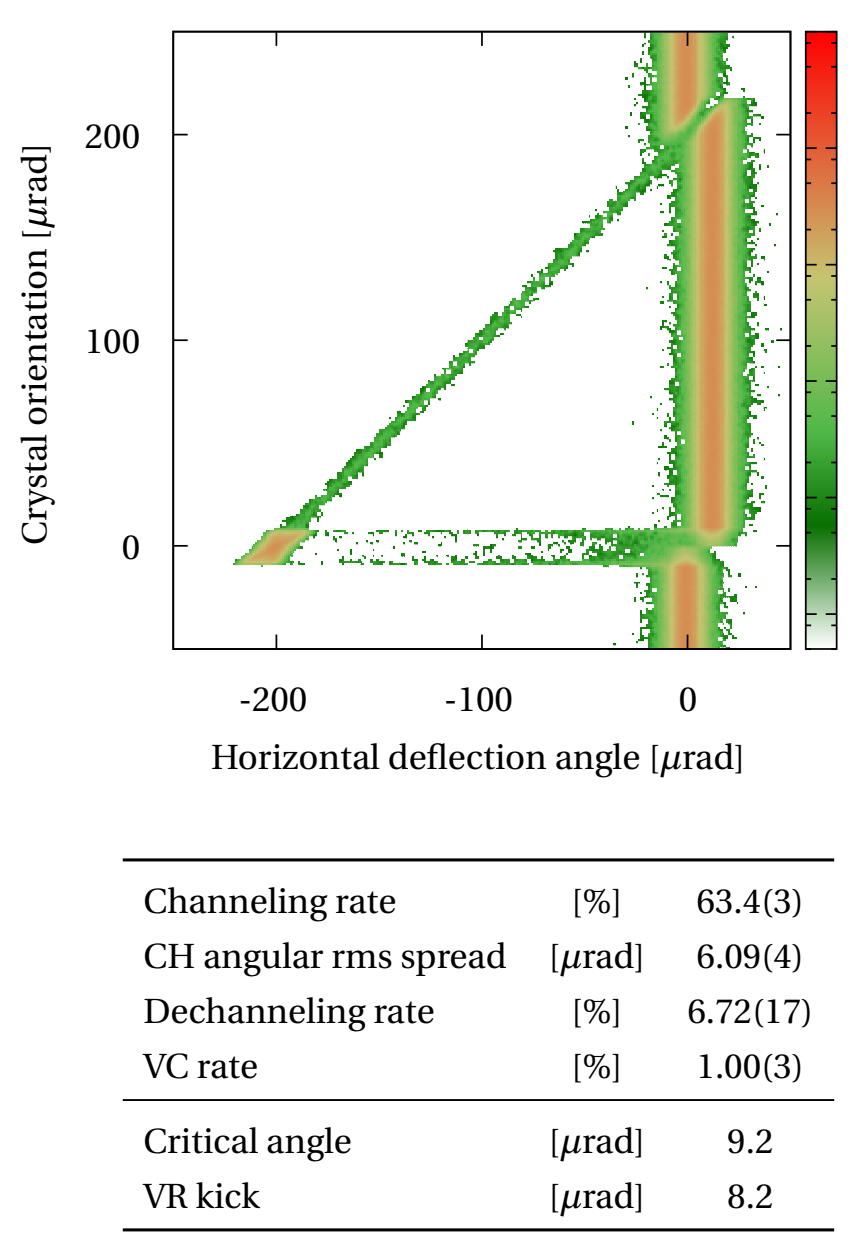

Fig. 3.17. Beam distribution as a function of the crystal orientation and horizontal deflection angle for the base scenario (case 0) along with key figures. 
CH rate: Percentage of particles being channeled (when the crystal is in channeling orientation). Here, channeled means that the particle has acquired a kick within $3 \sigma$ from the mean channeling deflection and channeling orientation means crystal orientations within $\left[-\theta_{c}, \theta_{c}\right]$. The maximal channeling rate obtained in ideal conditions is the channeling efficiency.

Channeling particles angular spread : Standard deviation of the angular distribution of channeled particles at crystal exit. This definition requires to know which are the channeled particles. A converging iteration is done to determine the angular spread and the corresponding channeling rate.

Dechanneling rate: Percentage of particles being dechanneled for a crystal in channeling orientation.

VC rate : Percentage of particles being captured in the crystal channels in VR orientation.

Critical angle: Typical amplitude of the angular half-aperture of a channel (parameter of the crystal).

VR average kick: Value of the kick given through the reflection process. It derives from the critical angle, as per 2.34, and differs from the total kick received by the reflected particle through the MCS contribution across the crystal.

In the present case, the channeling rate is large, at $63 \%$. As the beam divergence is zero, the only limitation to channeling is the centrifugal effect due to the crystal radius of curvature and the dechanneling process. The dechanneling rate is also high, as it is scales with the channeling rate. The root-mean square (rms) angular spread, obtained through sampling of an exit orientation out of the channeling oscillation is close to the value of the critical angle.

In fig. 3.17, the region populated by channeled particles is tilted and presents the same inclination that the volume capture region. This is caused by the absence of divergence of the beam. In this case, the channeling kick depends on the incoming angle of the channeling particle. Indeed, when the particle becomes channeled, the information about its angle prior to the trapping is lost and the resulting kick given by the crystal naturally includes additionally the value of that angle. This is an exception to what has been said above about our representation being supposed to yield an "untilted" channeling spot. Indeed, as there is no divergence, $\theta_{\text {in }}=\overline{\theta_{\text {in }}}$ and the adopted representation of the horizontal deflection of the particle $\theta_{\text {out }}-\theta_{\text {in }}$ is equivalent to the representation of fig. 2.11 where the quantity on the $x$-axis is $\theta_{\text {out }}-\theta_{\text {in }}$. It is therefore logical to obtain a similar orientation of the channeling spot. 


\subsubsection{Case 1 : Beam with large divergence}

In this first spin-off scenario, we assume all parameters as in the previous case, except the horizontal divergence : $\sigma_{x^{\prime}}=10 \mu \mathrm{rad}$. Representing a divergent beam as a function of the mean incoming angle (or crystal orientation) gives a stretching along the respective axis of the graph as we explained earlier. In fig. 3.18, the channeling spot has the same extension along the deflection angle axis but is much longer in the crystal orientation dimension leading to the reduction of the tilt of the spot. As a consequence, the separation between the regimes is harder to define because, for some crystal orientations, there are at the same time channeled/dechanneled particles and reflected/captured particles. This case is much closer to reality where the ideal orientation is not known in absolute terms. It is then defined as the one maximizing the channeling.

There, the channeling rate appears strongly reduced as a lower fraction of particles has an incoming angle small enough to be channeled, even when the crystal is ideally aligned. The dechanneling rate is reduced accordingly whereas the other figures are similar to the base scenario. 


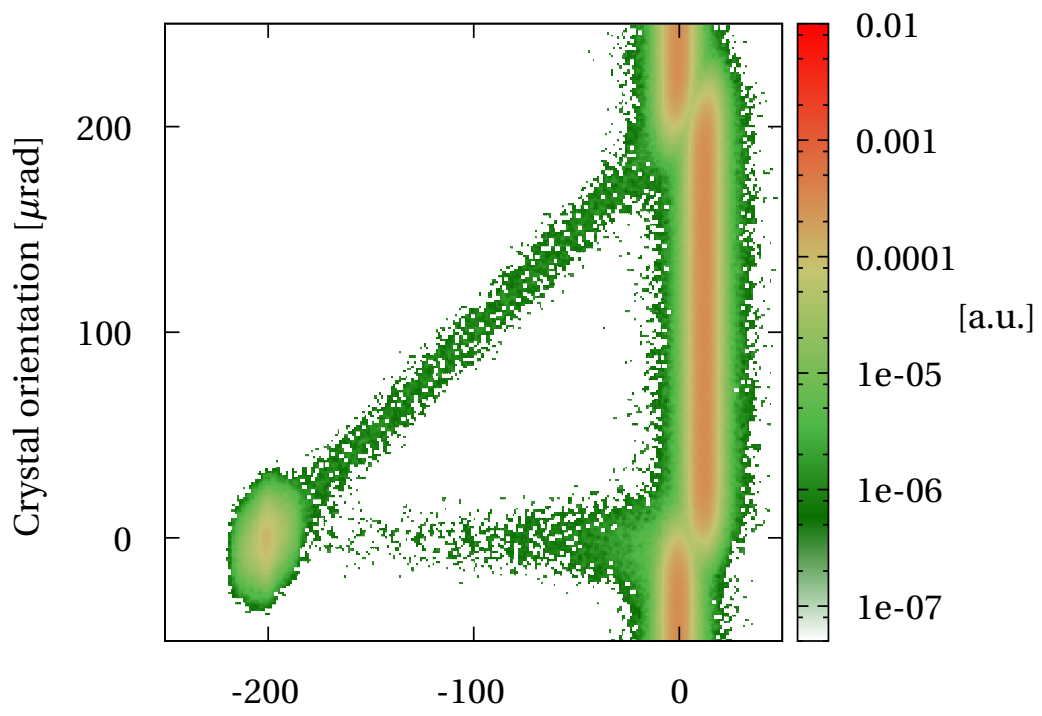

Horizontal deflection angle $[\mu \mathrm{rad}]$

\begin{tabular}{lcc}
\hline Channeling rate & {$[\%]$} & $39.1(3)$ \\
CH angular rms spread & {$[\mu \mathrm{rad}]$} & $5.45(3)$ \\
Dechanneling rate & {$[\%]$} & $3.27(9)$ \\
VC rate & {$[\%]$} & $0.95(2)$ \\
\hline Critical angle & {$[\mu \mathrm{rad}]$} & 9.2 \\
VR kick & {$[\mu \mathrm{rad}]$} & 8.2 \\
\hline
\end{tabular}

Fig. 3.18. Beam distribution as a function of the crystal orientation and horizontal deflection angle for the large divergence case (case 1) along with key figures. 


\subsection{3 | Case 2 : Strong torsion}

After having treated the main beam imperfection, the case of a twisted crystal is now considered. Though torsion can now be kept under a few $\mu \mathrm{rad} / \mathrm{mm}$ in the most recent crystals, it is interesting to see the consequences of a strong torsion. The one illustrated here corresponds to the highest measured value in the UA9-H8 experimental runs : $10 \mu \mathrm{rad} / \mathrm{mm}$

The beam has a spread in the vertical direction of $\sigma_{y}=1 \mathrm{~mm}$. Under these conditions, a torsion of $10 \mu \mathrm{rad} / \mathrm{mm}$ can substantially change the channel orientation over the dimensions of the beam. For a given crystal orientation, this increases the incoming angle range of beam particles and is comparable to the effects of increased divergence. Figure 3.19, displays the results of the strong torsion case. The channeling spot is shown to be close to the one seen in the previous case and the values given in the respective table are very close to the large divergence case.

\subsection{4 | Case 3 : Short crystal (Small bending radius)}

The following cases are conducted with intermediate values for the $x$ divergence and the torsion ( $5 \mu \mathrm{rad}$ and $2 \mu \mathrm{rad} / \mathrm{mm}$ respectively) which are closer to real cases met in test beams.

The smaller the crystal radius of curvature, the more the crystal deflects the particles per unit length. The price to pay for this increased performance is a loss of efficiency because of the increasing importance of the potential centrifugal term. This third case shows the results of the model when the base scenario is modified by shortening the crystal and its radius of curvature to one fifth of their base length. This keeps the bending angle unchanged at $200 \mu \mathrm{rad}$.

Due to the reduced radius of curvature, incoming particles must comply with a harder condition in order to be channeled. The barrier height and the critical angle are lower than in the previous cases. In addition, the potential is strongly asymmetric due to the centrifugal effect and the sampling of the potential energy is then more likely to yield high values. This can be understood (in the $7 \mathrm{TeV} / \mathrm{c}$ case) comparing the red and yellow curves in fig. 3.16. Therefore, the channeling rate is drastically reduced. The channeling zone is more confined which results in a reduction of the volume reflection

kick. In a short crystal, the rms kick due to MCS is smaller, as displayed by the nonchanneled particles exit angle distribution which has become narrower. 


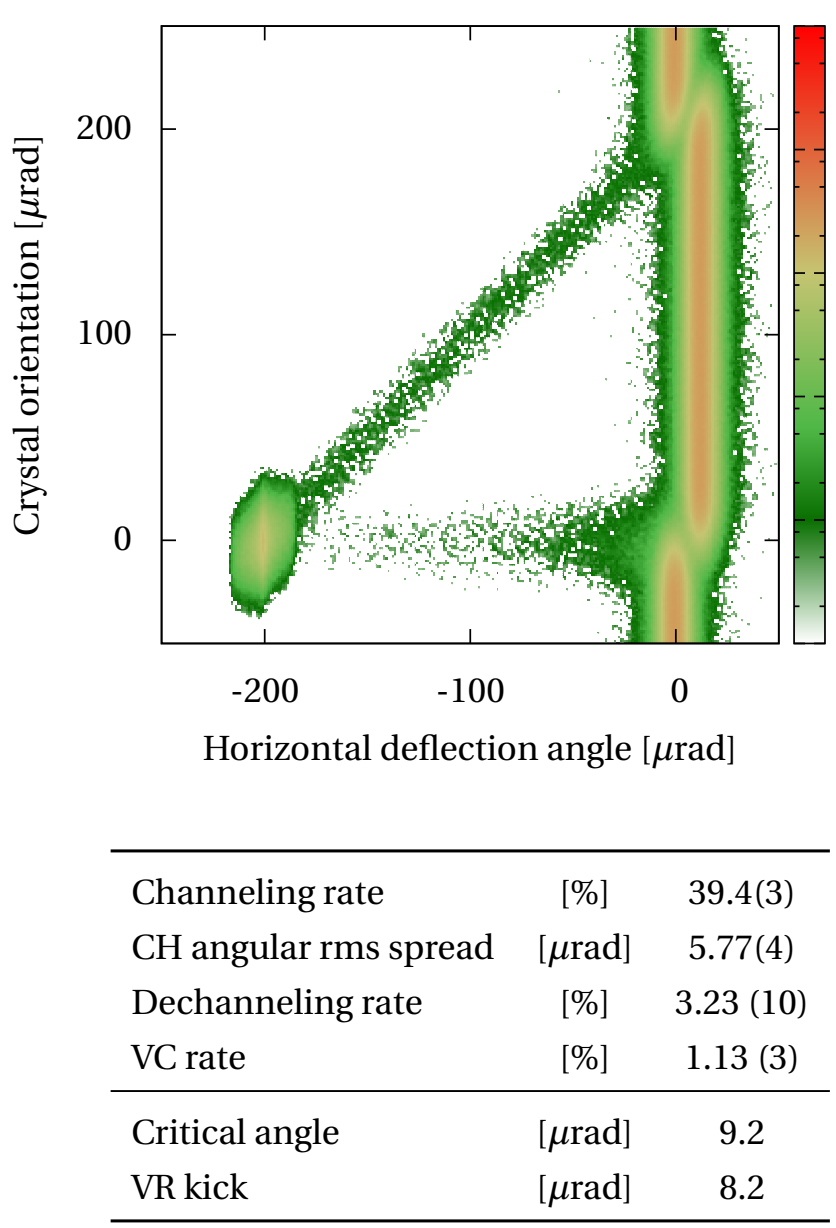

Fig. 3.19. Beam distribution as a function of the crystal orientation and horizontal deflection angle for the strong torsion case (case 2) along with key figures. 


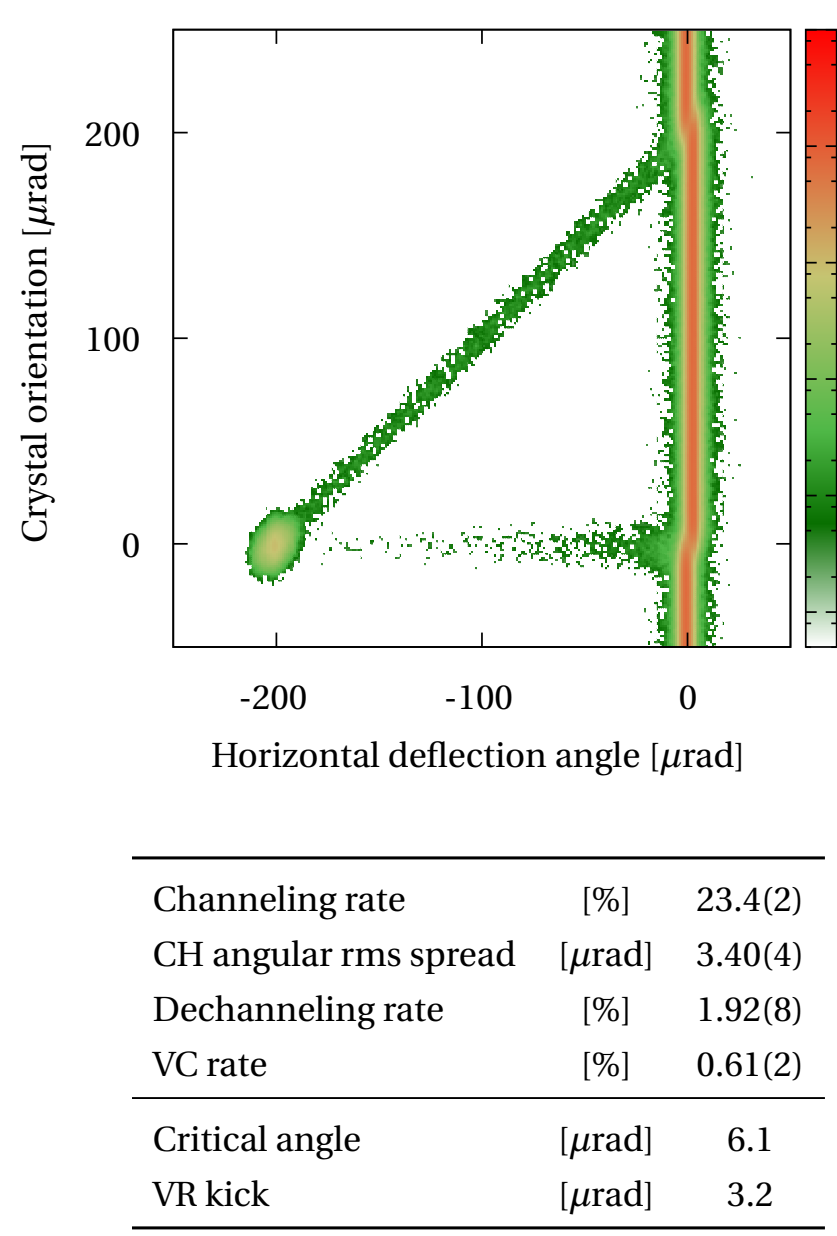

Fig. 3.20. Beam distribution as a function of the crystal orientation and horizontal deflection angle for the short crystal/radius case (case 3) along with key figures. 


\subsubsection{Case $4: 7 \mathrm{TeV}$}

For the moment, only protons at $400 \mathrm{GeV}$ have been treated. Testing a proton beam at a different energy or a beam composed of particles different than protons tells much about the model. The last two cases are conducted with $7 \mathrm{TeV}$ protons and $400 Z \mathrm{GeV}$ lead ions respectively.

In this case, a proton beam with an energy of $7 \mathrm{TeV}$ is sent onto the crystal. At such a high energy, the centrifugal term becomes much more important because of the dependence in $p$. The centrifugal term goes to $70 \mathrm{eV} / \AA$ for $R=10 \mathrm{~m}$, and completely suppresses the potential well. In reality, crystals prepared for LHC present a bending angle of 40 to $60 \mu \mathrm{rad}$ corresponding to a radius of curvature $R=40 \mathrm{~m}$ or $33 \mathrm{~m}$ respectively, which is sufficient to deflect particles with a suitable impact parameter onto the secondary collimator. Here the bending angle is decreased to $50 \mu \mathrm{rad}$. Consequently, the centrifugal term is $17 \mathrm{eV} / \AA$ ( 4 times more than in the previous cases). The critical angle for a $7 \mathrm{TeV}$ case is $2.4 \mu \mathrm{rad}$ for a straight crystal, and goes down to $1.5 \mu \mathrm{rad}$ for the bending radius $R=40 \mathrm{~m}$.

Figure 3.21 summarizes the results of the $7 \mathrm{TeV}$ case. As the critical angle is small, the channeling region is narrow. The channeling rate is low because of the small critical angle and the strong asymmetry of the effective potential (see previous section).

The VR kick value is smaller than expected. As explained in p. 20, the geometrical model used to calculate the one-time kick given in the reflection process makes the approximation of a long radius of curvature, usually acceptable when the radius is larger than 3 times the critical one. This case illustrates a bending which is well in the range of those investigated as suitable for the LHC. However the critical radius is about $40 \mathrm{~m}$ and the geometrical model shows a limitation in the present case.

\subsection{6 | Case 5 : Lead Ions}

The model has been developed in order to account for any type of positively charged beam. Ions can be channeled in the same way as protons. The Moliere potential scales with the charge number $Z$ of the incoming particle but its transverse energy obviously scales with $A$. In that regard, the channeling condition in a straight crystal is equivalent for protons with momentum $p_{\mathrm{p}^{+}}$and for ions with the same magnetic rigidity, having a momentum :

$$
p_{\text {ion }}=Z_{\text {ion }} p_{\mathrm{p}^{+}}
$$

where $Z_{\text {ion }}$ denotes the charge number of the considered ion. However, in a bent crystal, it has to be underlined that the centrifugal component increases with the total momentum of the particle whereas the Moliere potential scales with $Z$. As a corollary, the 
importance of the centrifugal effect is increased by a factor 2.5 for lead ions and despite a weaker bending, the critical angle is smaller.

In figure 3.22, the results for the lead ions case are displayed. The lower momentum per nucleon $158 \mathrm{GeV} / \mathrm{c}$ compensates the naturally smaller rigidity of ions. The higher channeling rate reflects the choice of a smaller bending. If the same bending had been chosen, the rate would be essentially the same as for $400 \mathrm{GeV} / \mathrm{c}$ protons. Dechanneling and volume capture are slightly more likely to happen, due to the ions interacting more frequently with the medium. In channeling, the momentum transfers obtained through application of the form factor is expected to span higher values. As a consequence the fraction of particles susceptible to be dechanneled (which requires a transverse energy high enough at the start) is higher. Of course, energy transfers are as much increasing the transverse energy as they are decreasing it thus the global increase in the rate is not spectacular. 


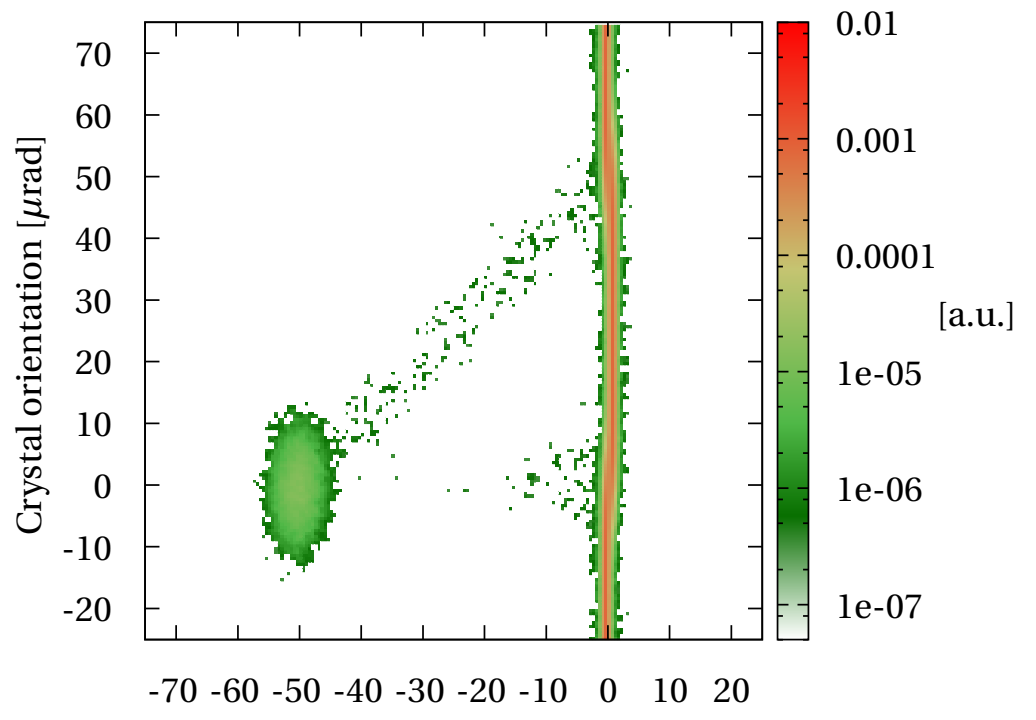

Horizontal deflection angle $[\mu \mathrm{rad}]$

\begin{tabular}{lcc}
\hline Channeling rate & {$[\%]$} & $9.08(40)$ \\
CH angular rms spread & {$[\mu \mathrm{rad}]$} & $1.89(3)$ \\
Dechanneling rate & {$[\%]$} & $0.89(4)$ \\
VC rate & {$[\%]$} & $0.62(4)$ \\
\hline Critical angle & {$[\mu \mathrm{rad}]$} & 1.6 \\
VR kick & {$[\mu \mathrm{rad}]$} & 1.6 \\
\hline
\end{tabular}

Fig. 3.21. Beam distribution as a function of the crystal orientation and horizontal deflection angle for the $7 \mathrm{TeV}$ case (case 4) along with key figures. Note the different angular scale with respect to the previous cases. 


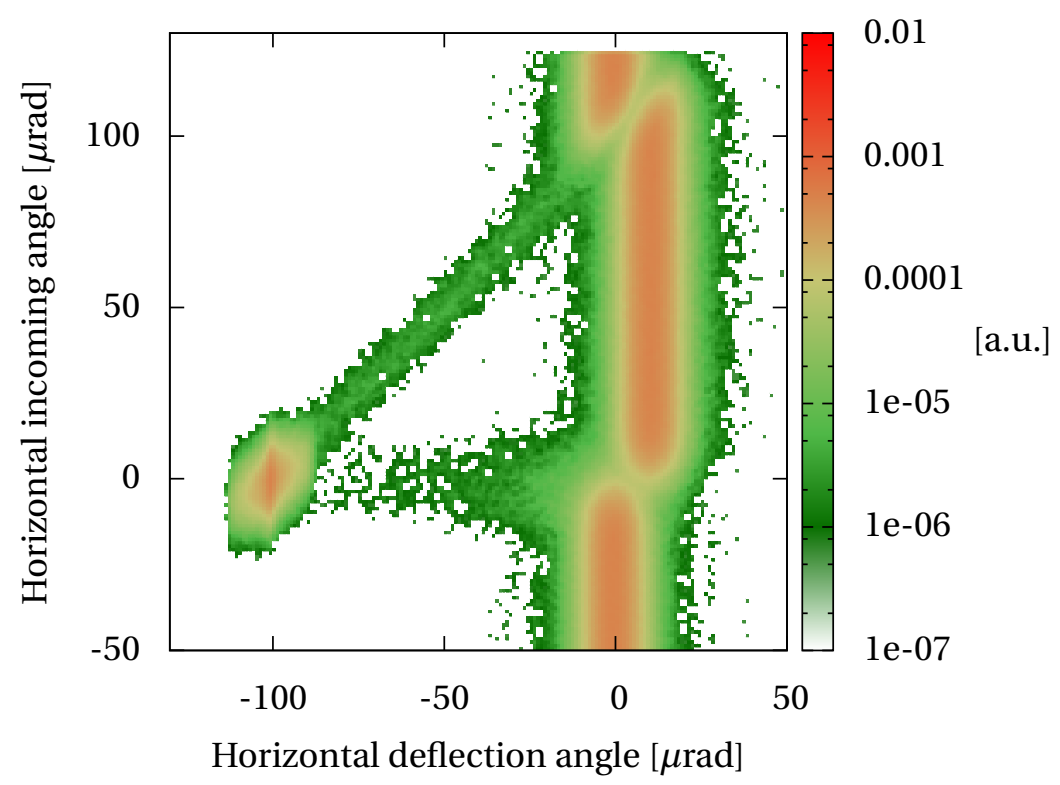

\begin{tabular}{lcc}
\hline Channeling rate & {$[\%]$} & $51.9(3)$ \\
CH angular rms spread & {$[\mu \mathrm{rad}]$} & $4.63(2)$ \\
Dechanneling rate & {$[\%]$} & $2.81(7)$ \\
VC rate & {$[\%]$} & $1.11(3)$ \\
\hline Critical angle & {$[\mu \mathrm{rad}]$} & 7.4 \\
VR kick & {$[\mu \mathrm{rad}]$} & 9.6 \\
\hline
\end{tabular}

Fig. 3.22. Beam distribution as a function of the crystal orientation and horizontal deflection angle for the lead ions case (case 5) along with key figures. 



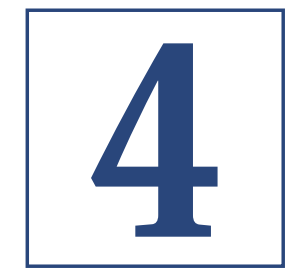

\section{FOURTH CHAPTER}

\section{EXPERIMENTAL ACTIVITY IN CERN NORTH} AREA

\subsection{Introduction}

UA9 is an international collaboration supported by CERN, INFN (I), PNPI, IHEP, JINR (RU), Imperial College (GB) and LAL (F). It is conducting 3 experiments at the CERN accelerator complex. The first one is located in the North Experimental Hall, on a beamline extracted from the SPS. The $400 \mathrm{GeV} / \mathrm{c}$-proton incoming beam impacts a crystal placed on a goniometer. Particle trajectories are measured thanks to a telescope composed of 5 silicon tracker planes. Such a setup allows to assess crystals performance and characteristics. In the meantime, the behavior of crystals in multi-turn operation is analyzed at the SPS, using coasting beams of 120 or $270 \mathrm{GeV} / \mathrm{c}$. The multi-turn halo (particles escaping the collimation system and coming back to it in a later turn) as well as the off-momentum halo (particles escaping with a small momentum offset and being lost in a dispersion region) are measured by bringing absorbers towards the center of the beam and recording losses on BLM, scintillators or Cerenkov detectors. The longerterm goal is to improve the existing LHC collimation system (see chapter 1) for which the experimental activity is ongoing and due to begin its data taking after the current long shutdown.

This chapter will be devoted to a detailed description of the North Area experiment, hereafter referred to as UA9-H8, from the name of its beamline. The direct application of the model discussed in the previous chapter to this single-pass experimental layout explains that choice. We describe the experimental layout and the different types of hardware providing the data. 


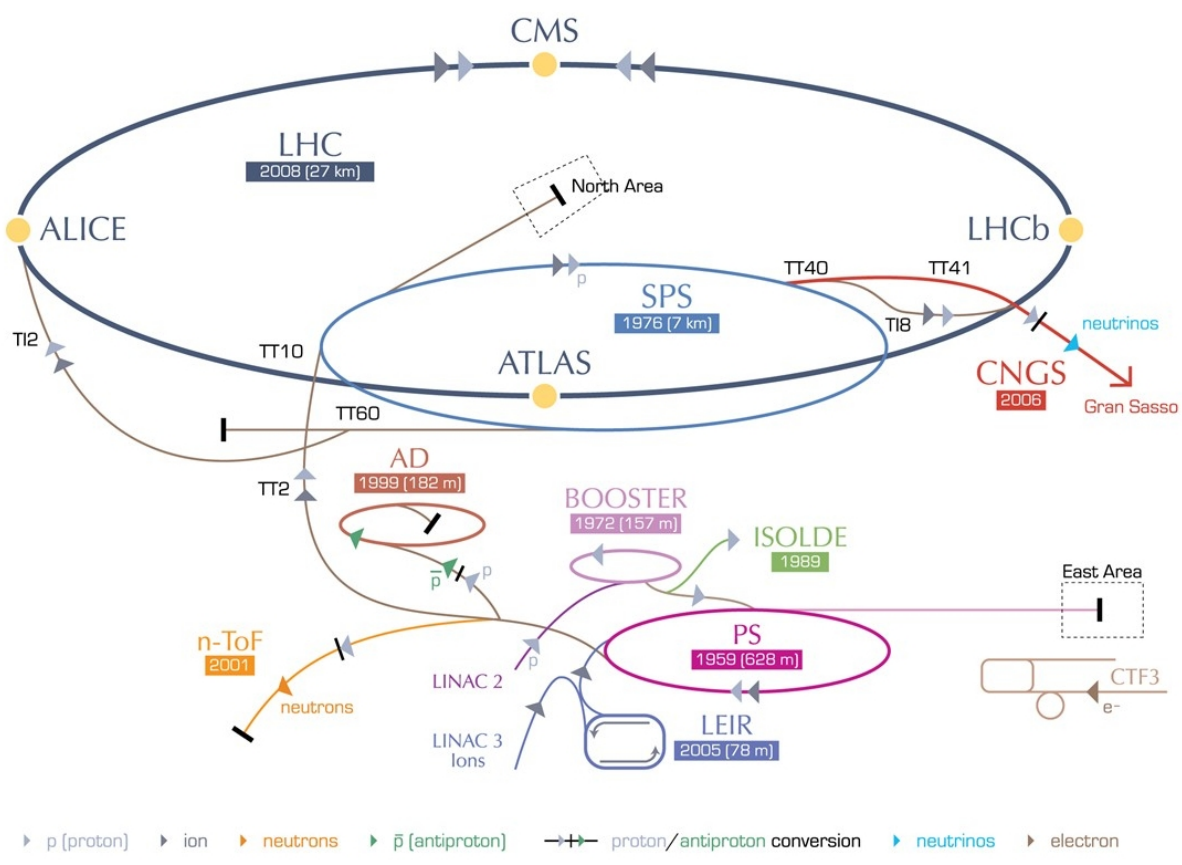

Fig. 4.1. CERN accelerator complex. The SPS, the second accelerator energy-wise, as well as the North Area, shown schematically at the top part of the figure, host the UA9 experiments. Next year, a crystal experiment will be installed in the LHC. Image courtesy of CERN.

\subsection{Providing beams to the North Area}

\subsection{1 | CERN accelerator complex}

The accelerator complex situated at CERN provides scientists around the world with data from reactions taking place over the widest energy range. Fig. 4.1 sketches the different machines and experimental areas in operation at CERN.

The UA9 experiment is installed on the H8 beamline. Protons or lead ions are extracted into this beamline from the SPS, the second biggest accelerator in operation at CERN injecting beams into the LHC and providing beams to many experiments (e.g. COMPASS, CNGS,...). This accelerator uses the Proton Synchrotron (PS) complex, itself composed of the LINAC, the (PS) Booster and the PS as injector. Protons arrive in the SPS with a momentum of $26 \mathrm{GeV} / \mathrm{c}$ and are accelerated up to $p=450 \mathrm{GeV} / \mathrm{c}$ before extraction into the LHC. However, beams extracted towards the North area are limited to $400 \mathrm{GeV} / \mathrm{c}$ so that the SPS can sustain a longer slow-extraction process leading to longer spills (with a lower amount of particles per time unit) for the experiment. 


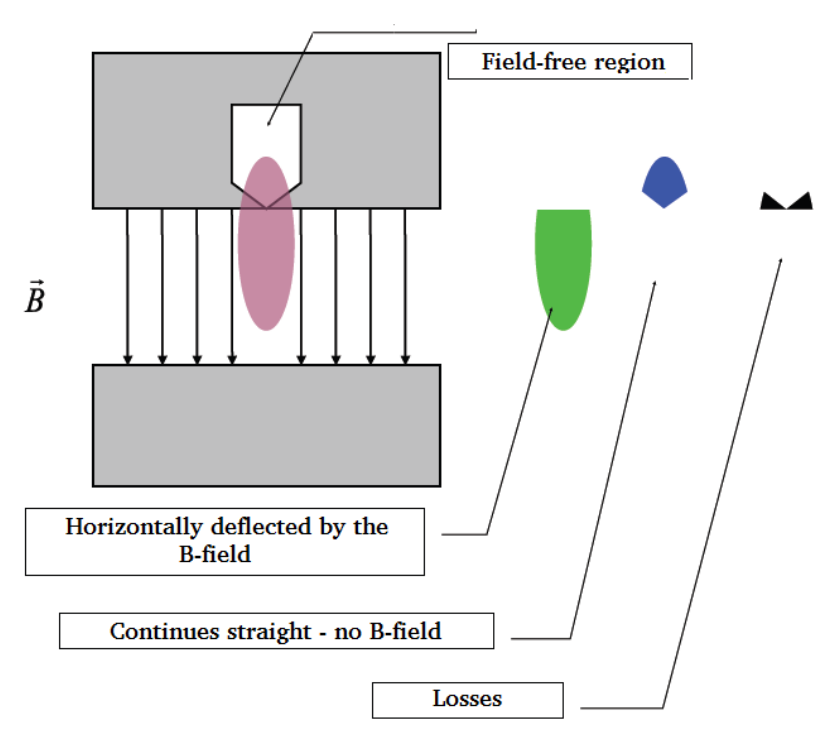

Fig. 4.2. Sketch of the septum magnet extracting particles from the SPS towards the North Experimental Area. The part of the beam passing through the shielded field-free zone is not deflected and is separated from the beam. Losses cannot be totally avoided in the process. Courtesy I. Efthymiopoulos

\subsubsection{Extraction from the SPS}

In the SPS, a septum is used to perform a slow extraction of primary particles towards the TT20 extraction line going towards the North Area. With this technique, each spill lasts for about $30 \mathrm{~s}$ in the experimental installations. The septum magnet, sketched in fig. 4.2, divides the beam into two parts. The particles passing in the aperture are deflected by the nominal field, keeping them in the orbit of the accelerator. A second part is passing in the shielded zone without magnetic field. These particles separate from the beam and are transported to the transfer line. Moreover, some beam particles pass through the bulk of the magnet and interact with it, which leads them to be lost in the vicinity. For this reason, the surrounding area is strongly activated. At the septum location, $\beta_{x}$ is very small $(\sim 9 \mathrm{~m})$ and $\beta_{y}$ is very large $(\sim 23 \mathrm{~km})$ in order to minimize the losses.

\subsubsection{The TT20 line to the North Area}

The extracted beam is then conveyed to the target switchyard TCC2 where it is split again into 3 beams feeding different target stations downstream. This allows an increased flexibility for the experiments as each of the target stations can be tuned independently. Downstream the target, a shower of secondaries is obtained and an absorber is placed just behind. This absorber includes moving parts that filter the secondaries according to the needs of the experimentators. Each target station has more than one secondary beam and wobbling magnets are used to deflect the beams to their corresponding destination (see fig. 4.3). For example, the T2 target station feeds the H2 and $\mathrm{H} 4$ beamline and the T4 target station feeds the H6, H8 and PO beamlines. After the absorber, further particle selection is done through the use of a secondary target and subsequent electrostatic and radio-frequency separation processes. Finally, the optics of the 


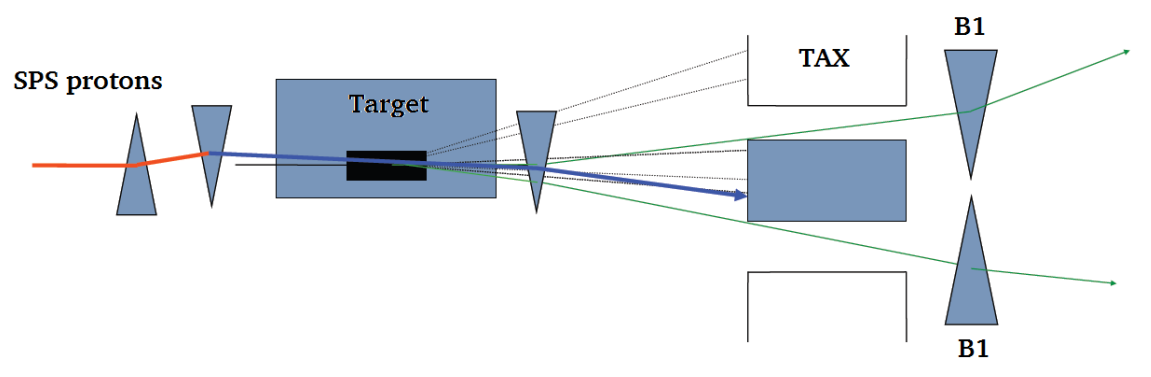

Fig. 4.3. Schematic layout of the target stations in TCC2. The wobbling magnets (around the target) add degrees of freedom to the orientation of the secondary beam. The absorber (TAX) is placed behind the target and filters the secondaries according to the needs of the experiment. The magnets B1 deflect the different beams towards different beamlines in the experimental hall. Courtesy I. Efthymiopoulos

line leading to the EHN1 (or North) experimental hall can be tuned by the experiment members to select the momentum of the secondaries, reduce the divergence and adjust the position.

The H8 beamline is a high-energy beamline capable of providing protons from 10 to $450 \mathrm{GeV} / \mathrm{c}$ and up to $400 \mathrm{GeV} / \mathrm{c}$ per charge primary lead ions. A typical value of the dispersion is $\Delta p / p= \pm 1.5 \%$. For a burst of $10^{12}$ protons incoming on the T4 target, intensities of about $10^{7}$ protons at $400 \mathrm{GeV} / \mathrm{c}$ can be attained in the experiment.

\subsection{The UA9-H8 experiment}

The experiment set up by the UA9 collaboration in the H8 beamline aims at characterizing crystals properties in view of their future installation in the SPS, where they can be in turn tested in a synchrotron environment, or in the LHC, where the crystal collimation experiment is in preparation.

Experimental runs have been conducted exclusively with protons. Technically, ion beam interaction with crystals could also be studied and this will be one of the goals of the experiment in the future. The beam incoming onto the crystals had a divergence in $x$ and $y$ of about $10 \mu \mathrm{rad}$ which at $400 \mathrm{GeV} / \mathrm{c}$ allows a fraction of the incoming particles to enter channeling regime. The experimental setup is installed in two zones along the H8 beamline. In the upstream zone, 5 tracker planes are placed on both sides of a goniometer. A scintillation counter serves as trigger for the data acquisition system (DAQ). The counter can be placed upstream or downstream. In front of the telescope, it adds uncertainty to the particle direction, slightly degrading the measurement. If placed behind it, the triggering process is altered by the secondaries created when the beam crosses the 
planes. Eventually, both setups have been used during the past years. The trigger was placed downstream when the focus was put on the study of nuclear interactions (multiple tracks downstream the crystal). In the second zone, about 40 meters downstream of the goniometer, various additional detectors can be installed typically allowing a live visual monitoring of the presence of a channeled beam (e.g. thanks to a MEDIPIX). Indeed, at such distance the channeled beam is deflected by of several millimeters, making it distinguishable from the main beam.

\subsection{1 | Goniometer}

Crystals positioning in a high-energy particle beam requires state-of-the-art control tools in order to achieve the accuracy and repeatability needed for observing channeling effects. As mentioned in chapter 3, the critical angle for $400 \mathrm{GeV} / \mathrm{c}$ protons is $6.4 \mu \mathrm{rad}$. Without considering the divergence of the beam, angles of that order of magnitude separate a fully amorphous orientation from the optimal channeling one. At the LHC energy range, this angle goes down to $1.5 \mu \mathrm{rad}$ in a moderately bent crystal. This underlines the crucial need of accuracy in newer crystal channeling applications fulfilled by the use of a state-of-the-art goniometer.

The goniometer must be capable of reliably positioning crystals in their channeling orientation. The one used in the $\mathrm{H} 8$ setup is a commercial device from the Micos ${ }^{\circledR} \operatorname{com}$ pany. It consists of four independent stages providing motion control along 3 dimensions. They are positioned on top of each other in the following order, the tilt rotation stage being the uppermost :

- Standard linear stage moving the whole goniometer and the crystal along the horizontal transverse dimension.

- Main rotation around the vertical axis, typically used when an angular scan is conducted.

- A second linear stage whose role is to center the crystal working area exactly on the rotation axis so that a rotation does not create a parasitic transverse displacement of the device.

- Tilt rotation around the horizontal transverse axis. This stage is especially useful to verify the crystal structure regularity and investigate possible abnormal behavior.

Stepper motors used in each of the four stages provide good accuracy and repeatability values, summarized in table 4.1. The bottom two stages are illustrated in fig.4.4 where the stepper motors can be seen on the left side. The tilting stage of the goniometer is shown in fig. 4.5 with the motor on the right. The plate rotates around the horizontal axis perpendicular to the beam and graduations from $-5^{\circ}$ to $+5^{\circ}$ can be seen on the side. 


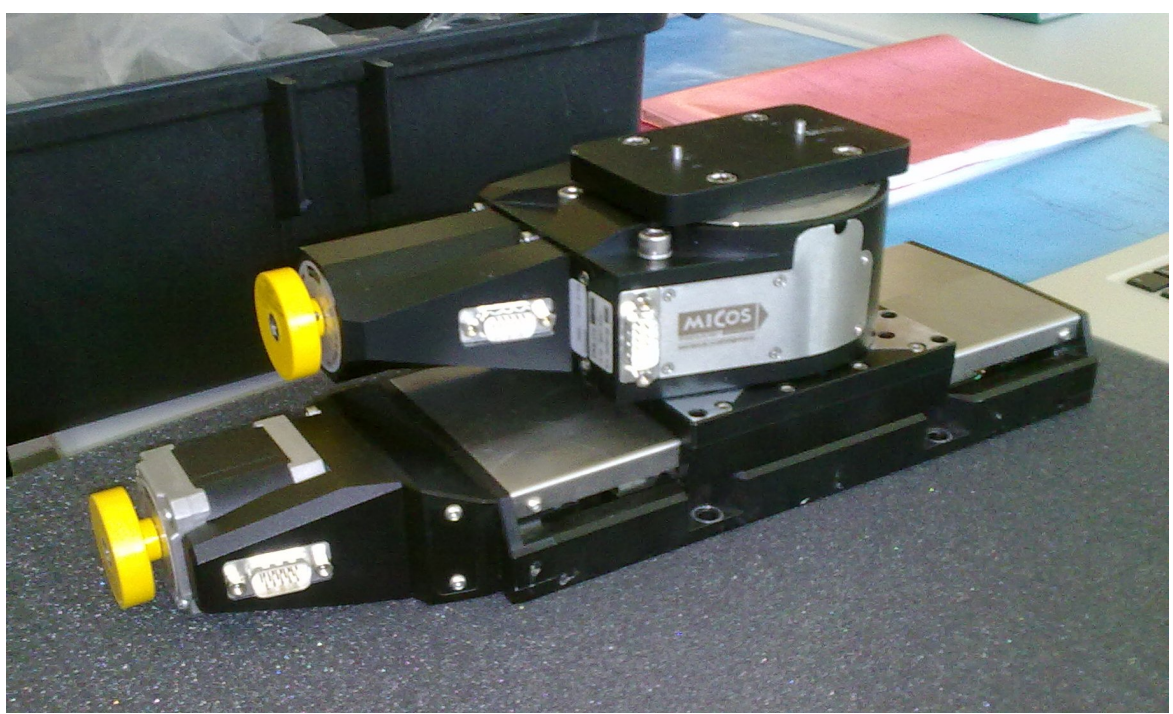

Fig. 4.4. Bottom part of the goniometer used in the UA9-H8 experiment, including the linear stage and the rotation stage of the goniometer used in the H8 experiment : movement along $x$ (bottom) and rotation about the vertical axis (top).

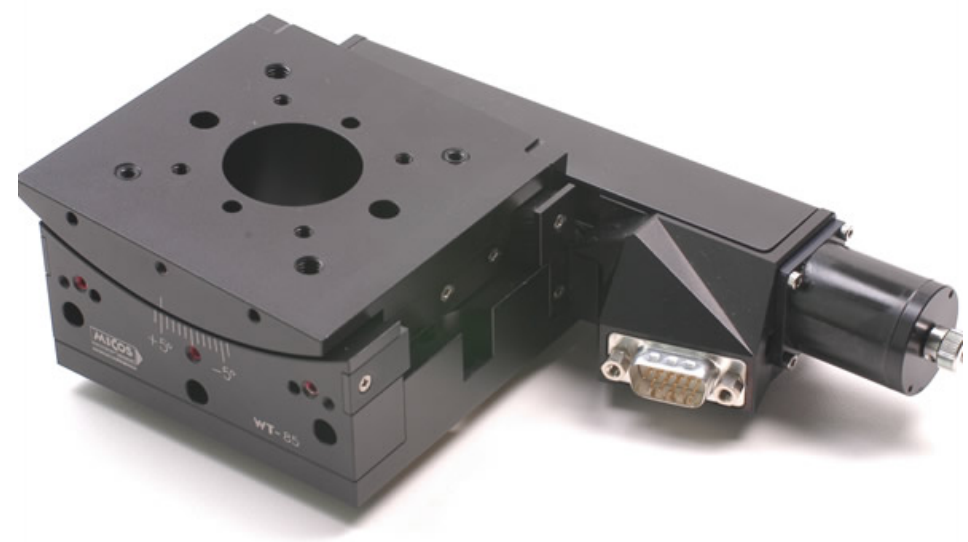

Fig. 4.5. Tilting stage of the goniometer (CMICOS). 
Table 4.1. Technical data of the goniometer stages. The repeatability value is crucial to the overall performance of the experimental setup. The value given is the one-way repeatability as, most of the time, care is taken to use the same movement direction when collecting data.

\begin{tabular}{lcccc}
\hline & TRAVEL RANGE & MAX SPEED & RESOlUtion & 1W-REPEAT. \\
\hline Linear stage (bottom) & $52 \mathrm{~mm}$ & $45 \mathrm{~mm} / \mathrm{s}$ & $10 \mu \mathrm{m}$ & $\pm 0.2 \mu \mathrm{m}$ \\
Main rotation stage & $360^{\circ}$, endless & $50 \% \mathrm{~s}$ & $3.4 \mu \mathrm{rad}$ & $\pm 3.4 \mu \mathrm{rad}$ \\
Linear stage (top) & $102 \mathrm{~mm}$ & $20 \mathrm{~mm} / \mathrm{s}$ & $5 \mu \mathrm{m}$ & $\pm 0.1 \mu \mathrm{m}$ \\
Tilt stage & $10^{\circ}$ & $7 \% / \mathrm{s}$ & $17 \mu \mathrm{rad}$ & $\pm 70 \mu \mathrm{rad}$ \\
\hline
\end{tabular}

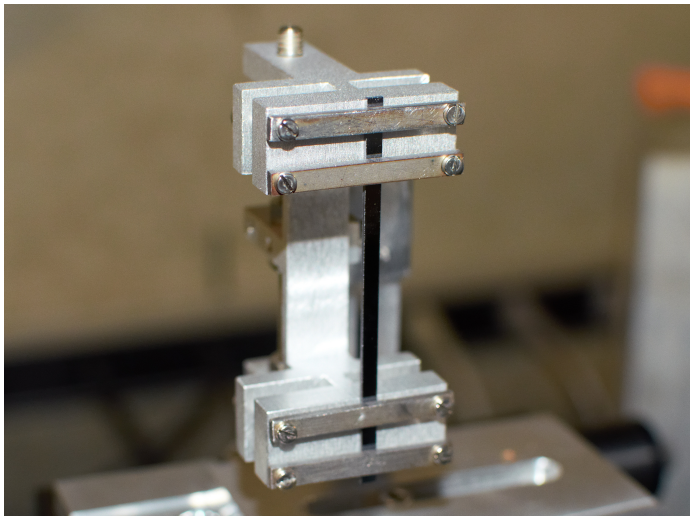

(a)

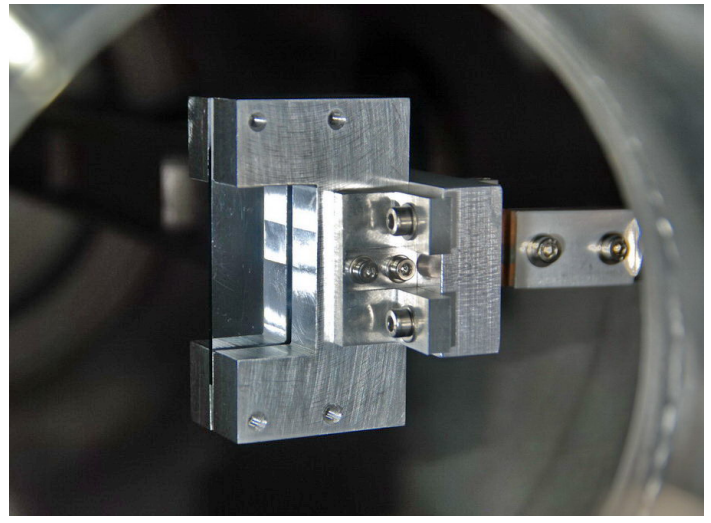

(b)

Fig. 4.6. (a) Strip silicon crystal in their aluminum holder. Screws seen on the strip crystal holder can be used to correct the torsion of the device. (b) Quasi-mosaic silicon crystal. The curvature used in channeling experiments is located along the smallest dimension.

\subsection{2 | Crystals}

Crystals are cut from a Silicon wafer or ingot. Scientists at INFN Ferrara have developed two methods of fabrication documented in [57] which use a combination of careful dicing with a $150 \mu \mathrm{m}$ diamond blade and chemical etching in order to produce a crystal with low mechanical damage and almost no dead layer ${ }^{1}$, especially near the lateral edges which are of critical importance for beam collimation purposes.

The current generation of crystals used in collimation experiments is characterized by a small thickness along the beam and a curvature imparted by the holder through mechanisms known as the anticlastic deformation or the quasi-mosaic effect. Fig. 4.6 shows the two types of crystals exploited by the UA9 collaboration in view of the experimental LHC crystal collimation experiment. The photograph on the left-hand side shows a strip crystal. Its edges are clamped to the holder, and the crystal is curved in the $x y$ plane. As a result, the strip takes the shape of a saddle and the deformation in the plane

\footnotetext{
${ }^{1}$ Damaged zone of a crystal where no coherent effect can take place due to chaotic crystalline structure.
} 
Fig. 4.7. Anticlastic curvature in a bent strip crystal. The holder bends the crystal along its long dimension, in the $x y$ plane, which causes the curvature in the $x z$ plane. Curvatures are indicated by an arrow (the radius of curvature). The dotted curve represents the intersection of the curvature plane with the crystal.

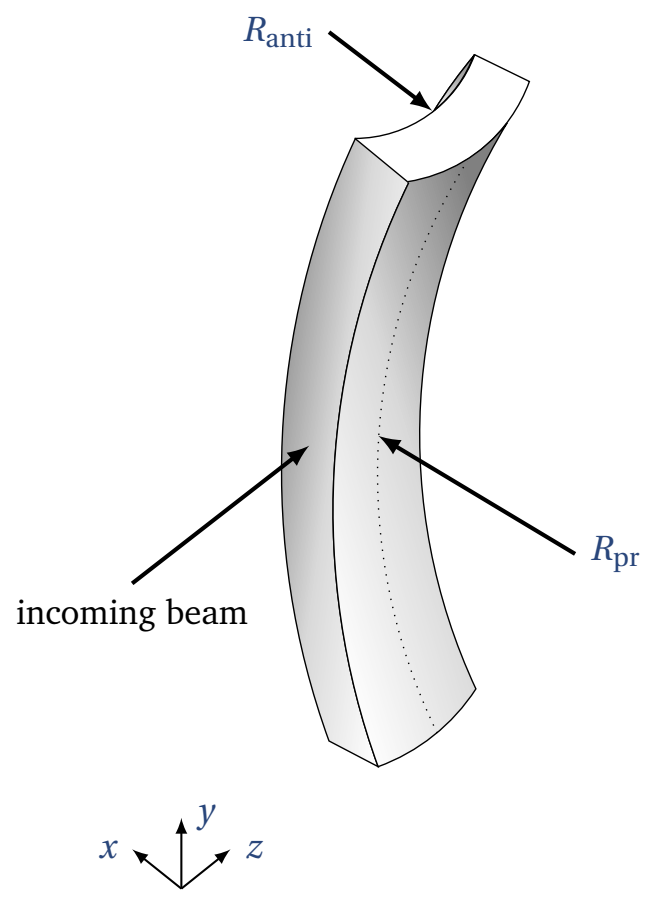

perpendicular to the primary one is called anticlastic curvature with a value proportional to the primary bending and inversely proportional to the Poisson's ratio of the considered material [58]. The resulting bending is schematically represented in fig. 4.7. On the right-hand side of fig. 4.6, the photograph shows a quasi- mosaic crystal in its holder. In this case, the crystallographic planes used for channeling are the ones parallel to the narrow face of the crystal (the one not enclosed in the holder). The holder creates a curvature in the $y z$ plane. In a crystal having a FCC symmetry, material properties are dependent on the orientation [59]. Therefore, in addition to the anticlastic deformation also present in this case, the primary curvature causes $y z$ sections of the crystal to be deformed to a parabolic shape which is called quasi-mosaic effect [57,60]. It is schematically represented in fig.4.8 where the primary curvature (radius $R_{\mathrm{pr}}$ ) is shown along with the anticlastic $\left(R_{\mathrm{anti}}\right)$ and quasi-mosaic one $\left(R_{\mathrm{qm}}\right)$. The latter is responsible for the bending of the channeled beam.

Both types of crystals were commonly used in UA9-H8 experiments as they both perform well in terms of channeling efficiency. An experiment where the crystal is put in the middle of the beam suffers however from the non-uniformity of the quasi-mosaic curvature along the transverse direction. For a crystal used as a collimator in a synchrotron, this effect is strongly reduced as particles interact only with the part of the device close to the beam. Other types of crystals as well as compound crystals were also tested in the scope of the experiment such as thin flat crystals allowing to investigate the PXR 


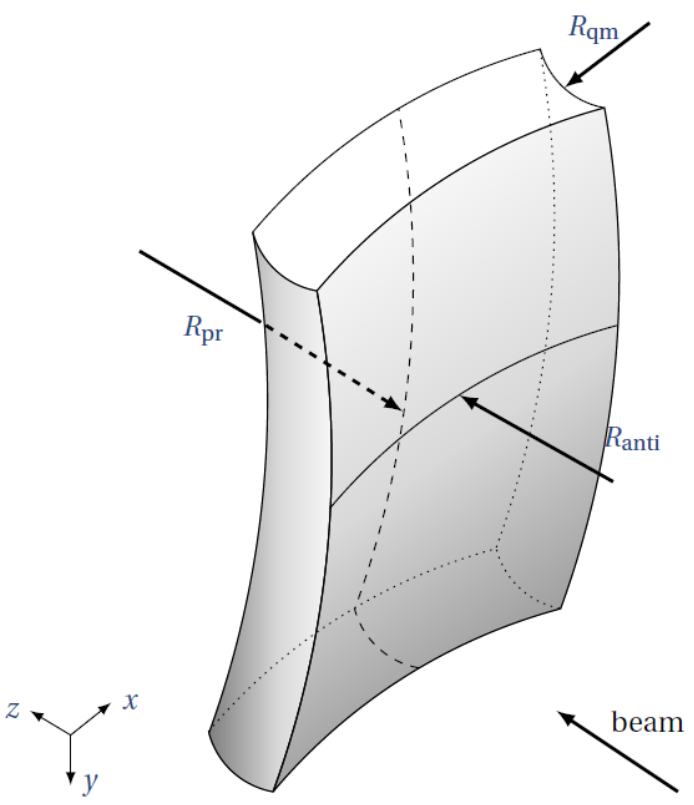

Fig. 4.8. Quasi-mosaic curvature (radius $R_{\mathrm{qm}}$ in the $x z$ plane) induced by the cylindrical bending of a Silicon plate in the $y z$ plane (radius $R_{\text {pr }}$ ). An anticlastic curvature (radius $R_{\text {anti }}$ ), perpendicular to the quasi-mosaic one, appears in the same plane $x z$.

effect $^{2}[61,62]$ or multi-strip crystals, consisting of several identical strips oriented so that a particle reflected from a given strip would also undergo volume reflection in the next ones [61, 63]. The model described in chapter 3 can in principle be applied for simulating such compound crystals, assuming a proper FLUKA geometry describing the crystal properties.

Table 4.2 gives the properties of crystals used in UA9-H8 experimental runs for which data has been analysed and the ensuing preliminary results are discussed in chapter 5 . These strip crystals, produced in Ferrara, have a typical length along the beam between 1 and $3 \mathrm{~mm}$ and a typical width close to $1 \mathrm{~mm}$. The quasi-mosaic crystals are produced in Saint-Petersburg and are up to $6 \mathrm{~mm}$ long and $2 \mathrm{~cm}$ wide.

\subsection{3 | Tracking planes - Data acquisition system}

The silicon telescope consists of 5 detectors placed around the goniometer as illustrated in fig. 4.9 representing the experimental layout. The first and last detectors are placed around 10 meters away from the goniometer in order to guarantee an angular resolution good enough for channeling studies, which at that energy, means few $\mu \mathrm{rad}$. Three other telescopes are placed close to the crystal location.

\footnotetext{
${ }^{2}$ Parametric X-rays or PXR is the diffraction process of the virtual-photon field of a fast charged particle on crystal planes leading to the emission of real photons.
} 
Table 4.2. Dimensions and bending angles of crystals used between 2010 and 2012 in the UA9-H8 experiment and considered for the data analysis. Strip crystals are manufactured at INFN Ferrara, Italy and quasi-mosaic at PNPI, Russia. The bending value is the one resulting from data analysis.

\begin{tabular}{lcccc}
\hline \multirow{2}{*}{ NAME } & \multicolumn{3}{c}{ DimENSIONS $[\mathrm{mm}]$} & $\theta_{b}$ \\
& Length $(\mathrm{z})$ & Width $(\mathrm{x})$ & Height $(\mathrm{y})$ & {$[\mu \mathrm{rad}]$} \\
\hline STF 47 & 3.1 & 2 & 55 & 35.5 \\
STF 48 & 2 & 1 & 55 & 142 \\
STF 49 & 0.8 & 0.5 & 55 & 245 \\
STF 50 & 2 & 1 & 55 & 138 \\
STF 51 & 3 & 2 & 55 & 37 \\
STF 70 & 3 & 1 & 55 & 54 \\
STF 71 & 3 & 1 & 55 & 61 \\
& & & & \\
QMP 26 & 2 & 18 & 50 & 50 \\
QMP 28 & 6 & 20 & 50 & 41 \\
\hline
\end{tabular}

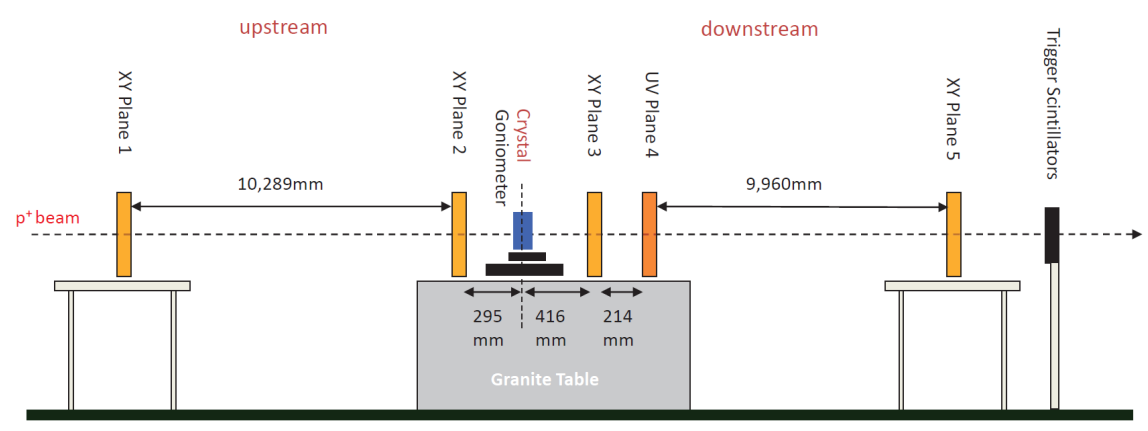

Fig. 4.9. Layout of the UA9-H8 experiment. The 5 planes of the tracking detector system are illustrated along with their position with respect to the crystal. They record the position of crossing particles in coincidence with the signal from the trigger counter, also indicated. Plane 4 is rotated by $45^{\circ}$ in order to disentangle multiple outgoing tracks in the reconstruction process [64]. 


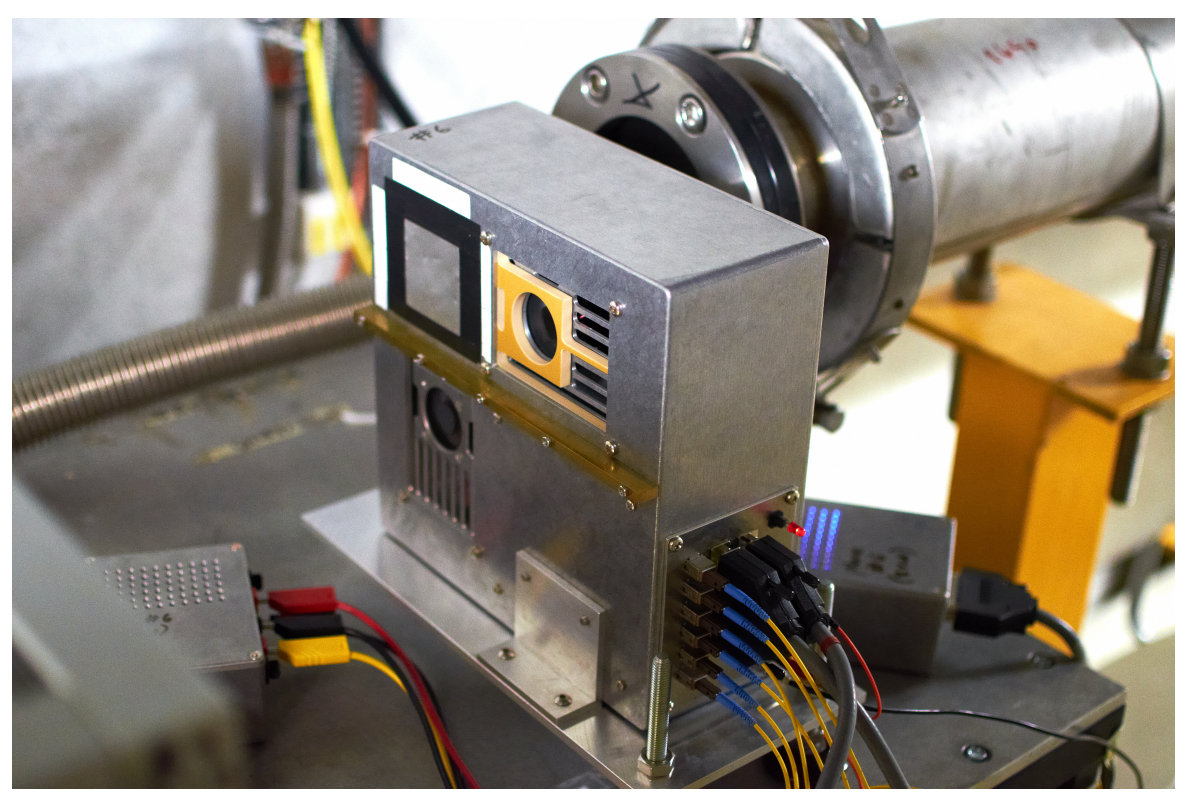

Fig. 4.10. Tracker plane used in the UA9-H8 experiment. The square on the top left of the plane corresponds to the active area of the silicon strip detector. Two fans and the optical fibers sending the signal to the DAQ can also be seen.

Comprehensive details about the high-resolution telescope are available in ref. [64]. Its most important features, along with measurement error values based on results from [65], are reminded here. The detector situated in each of the tracker planes, shown in fig. 4.10, is made of 2 silicon sensors, containing 639 strips overlapping in an active area of $38 \times 38 \mathrm{~mm}^{2}$, giving a readout pitch of $60 \mu \mathrm{m}$ in both dimensions. Intermediate floating strips help increase the resolution while keeping the same number of readout channels. Signals coming out of the strips are read out by APV25 chips, initially developed for the CMS spectrometer at the LHC [66]. The data is converted to an optical signal using a semiconductor laser and sent along single-mode optical fibers up to the control system, situated about 50 meters away, close to the control room. The use of fibers prevents electrical interferences and minimizes signal attenuation naturally arising from the use of long cables.

Crystals performance is evaluated by analyzing particle tracks crossing the crystal. The telescope returns couples of incoming/outgoing tracks that are found to hit the crystal at the same position. Track fitting also takes into account multiple scattering occurring on previous planes. Typically, a XY-plane containing two strip detectors (and the case) can be considered equivalent to $640 \mu \mathrm{m}$ of silicon and $60 \mu \mathrm{m}$ of aluminum. Monte Carlo simulations show that the rms deflection, projected on the horizontal plane, was $3 \mu \mathrm{rad}$ for the incoming arm and $5.4 \mu \mathrm{rad}$ for the whole telescope. These numbers are obtained assuming that air is removed from the system between plane 1 and 2 as well as between plane 4 and 5 . It is interesting to note that such a length in air $(2 \times 10 \mathrm{~m})$ 
would bring a major contribution to the global MCS process along the setup. Indeed, the radiation length in air is close to $300 \mathrm{~m}$ for protons at that energy (15 times the medium length) whereas it comes close to $10 \mathrm{~cm}$ for both Silicon and Aluminum (about 30 times the medium length).

The incoming track is a straight line between the hits on plane 1 and 2. The outgoing track corresponds to a straight line fit of the hit positions on planes 3, 4 and 5. A complete track is reconstructed when a pair of coincidental tracks yield the same hit position on the crystal. The admission criteria for a set of tracks in the reconstruction is that there must be at least one hit on each plane, and not more than 2 on a given plane. 


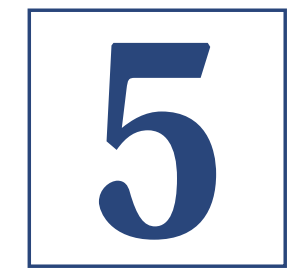

\section{FIFTH CHAPTER}

\section{BENCHMARKING AGAINST UA9-H8 DATA}

\subsection{Introduction to data analysis}

The UA9 experiment conducted in the North Area has been described in the previous pages. In this chapter, its results are analyzed and used for benchmarking the event generator. Data analysis is a crucial step of the work fulfilling two main objectives :

- Provide experimental values of interest that can be directly compared to the simulation. Each set of values, or "run", corresponds to specific crystal characteristics (dimensions, bending angle and torsion) and operation parameters (beam spread, position and divergence) and crystal orientation. The crystal characteristics are evaluated in the data analysis and then used as input of the simulation.

- Give an account of the crystal performance including efficiency figures corresponding to optimal alignment.

A run can correspond to different tasks undertaken during the experiment. It can contain the data taken with a crystal positioned in a given orientation (channeling, VR or amorphous) or during a scan (angular, lateral or tilt). It can also signify that an alignment procedure took place or that the DAQ was being checked. Not all runs are significant and, in this study, we are limiting ourselves to several runs where quasi-mosaic or strip crystals were placed in channeling orientation.

Crystals were placed in a $400 \mathrm{GeV} / \mathrm{c}$ proton beam and particle incoming and outgoing tracks were recorded thanks to 5 silicon strip detectors. Data from the experiment lists hit-positions on each of the five planes. The particle positions are calculated by linear interpolation of the charge collected between two adjacent strips. However, the charge deposition is actually non linear meaning that calculated hit positions are found to be 
situated preferentially close to the strips implants. As a consequence, a pattern can be seen on the beam distributions with a period of $30 \mu \mathrm{m}$ for the positions and a period $30 \mu \mathrm{m} / 10 \mathrm{~m} \approx 3 \mu \mathrm{rad}$ for the incoming angles. 95\% confidence intervals representing the statistical uncertainty of the data analysis are indicated via numbers in parentheses in tables 5.2 to 5.6. Incoming and outgoing tracks were reconstructed from the impact locations on the strip detectors, yielding hit coordinates on the crystal as well as inand outgoing angles of more than 7000 particles per second. In addition to the statistical uncertainty, the measurement error of the experimental setup is $2.8 \mu \mathrm{rad}$ for the incoming arm and $5.2 \mu \mathrm{rad}$ for the whole telescope. The error shown in the following graphs is the statistical error $\Delta_{s}$ which, together with the measurement error $\Delta_{m}$ gives the global uncertainty on the data $\Delta_{g}$ :

$$
\Delta_{g}=\sqrt{\Delta_{m}^{2}+\Delta_{s}^{2}}
$$

In the course of this chapter, crystals considered as candidates for crystal collimation are presented. First, the case of a strip crystal designed for the LHC will be analyzed. Then, a SPS strip crystal and a LHC quasi-mosaic crystal ${ }^{1}$ are discussed. Afterwards, an overview of other runs will be given along with a corresponding summary of the analysis outcome. The chapter ends with a discussion of the results, including ideas to further refine the event generator capabilities.

\subsection{Strip crystal for the LHC (STF 71)}

\subsection{1 | Beam characterization}

In this section, run 1191 conducted in October 2012 is going to be shown as an example of how we construct the benchmarking. This run used the STF 71 strip crystal manufactured by INFN Ferrara. Its dimensions are $L_{x} \times L_{y} \times L_{z}=1 \times 55 \times 3 \mathrm{~mm}^{3}$ and its nominal bending angle is about $60 \mu \mathrm{rad}$, especially suitable for the LHC where the radius of curvature must be relatively long in order to obtain an angular aperture compatible with the current resolutions of state-of-the-art goniometers. The crystal was placed into the $400 \mathrm{GeV} / \mathrm{c} \mathrm{H} 8$ proton-beam in channeling orientation.

Table 5.1 gives the beam parameters for each of the considered runs. The horizontal angle $x^{\prime}$, the vertical hit position $y$ and the vertical incoming angle $y^{\prime}$ distributions are close to Gaussian, and are described through their mean and standard deviation. For the incoming angles, only the orientation with respect to the crystal channels orientation is relevant. Therefore, the value indicated is the offset existing between the mean incoming

${ }^{1}$ LHC crystals have a bending angle typically smaller than $100 \mu \mathrm{rad}$, while crystals for the SPS have bending angles ranging up to about $200 \mu \mathrm{rad}$. 
Table 5.1. Characterization of the beam particles distributions in horizontal angle $x^{\prime}$, vertical position $y$ and vertical angle $y^{\prime}$. The mean incoming angles are indicated as offsets between the mean orientation of channeled particles, and the one of the whole beam. It is the value of interest used as input of the simulations.

\begin{tabular}{|c|c|c|c|c|c|c|}
\hline \multirow[b]{2}{*}{ RuN } & \multicolumn{2}{|c|}{$X^{\prime}$} & \multicolumn{2}{|c|}{$\mathrm{Y}$} & \multicolumn{2}{|c|}{$\mathrm{Y}^{\prime}$} \\
\hline & $\begin{array}{r}\text { Offset } \\
\mu\end{array}$ & $\begin{array}{l}\text { Sigma } \\
\text { Id }\end{array}$ & $\begin{array}{r}\text { Mean } \\
\mathrm{r}\end{array}$ & Sigma & Offset & $\begin{array}{l}\text { Sigma } \\
\text { d }\end{array}$ \\
\hline 608 & -1.46 & 14.11 & -0.03 & 1.00 & -0.04 & 10.63 \\
\hline 630 & -4.13 & 13.53 & 0.25 & 0.96 & 0.30 & 10.46 \\
\hline 650 & 0.96 & 14.68 & -0.05 & 0.96 & -0.07 & 10.34 \\
\hline 889 & 3.78 & 13.0 & -0.37 & 1.52 & -0.25 & 10.02 \\
\hline 899 & 0.49 & 13.1 & -0.06 & 1.54 & -0.08 & 10.00 \\
\hline 1012 & -2.95 & 9.38 & -0.21 & 0.83 & -0.01 & 6.15 \\
\hline 1028 & -3.24 & 9.72 & -0.08 & 0.84 & -0.04 & 6.17 \\
\hline 1191 & -2.53 & 9.69 & -0.30 & 0.84 & 0.08 & 6.40 \\
\hline 1240 & 0.04 & 9.62 & 0.08 & 0.85 & -0.01 & 6.43 \\
\hline
\end{tabular}

direction of the channeled particles distribution (assumed to coincide with the crystal ideal orientation) and the mean incoming direction of the whole beam.

Table 5.1 does not list any horizontal hit position parameter. Given the usual transverse dimensions of crystals (very thin along $x$ but long along $y$ ), the H8 beam size in $x$ (standard deviation of about $1 \mathrm{~mm}$ ) causes a substantial amount of particles to miss the crystal on its sides. Prior to the analysis, reconstructed tracks corresponding to a hit position on the left or on the right of the active area of the crystal ${ }^{2}$ are rejected. The beam parameters in $x$ are then irrelevant under the hypothesis that the crystal performance along $x$ is almost constant. In the simulations the transverse position is not exploited as an input parameter. For strip crystals, it is a good approximation as it will be demonstrated in the following. In the case of quasi-mosaic crystals though, this means that the anticlastic curvature is neglected.

\subsection{2 | 3D binning}

The DAQ provides a data reconstruction file, containing information about dual tracks consisting of an incoming and outgoing track having triggered at the same time and yielding a compatible hit position on the crystal. This includes incoming and outgoing angles, hit positions and deflection angles (all in both $x$ and $y$ ).

\footnotetext{
${ }^{2}$ In QM crystals, the active area is a zone of a few $\mathrm{mm}$ on the close edge of the device where the alignment procedure optimizes channeling.
} 
The data analysis is based on the classification of all tracks in bins along 3 dimensions :

- The horizontal hit position $x$ is only relevant for the purpose of crystal homogeneity determination (see above). The binning goes from one side of the crystal to the other. Their position are determined through preliminary analysis of MCS kicks given to particles.

- The horizontal incoming angle $x^{\prime}$. The binning covers tracks within 3 standard deviations from the mean.

- The vertical incoming angle $y^{\prime}$. Treatment similar to $x^{\prime}$.

Here, the vertical incoming angle is left out because its influence on the channeling rate is negligible. As it has been said earlier, the modification it can bring to the effective bending radius is very small and is unlikely to influence channeling. The binning should be fine enough so that the resolution on the channeling rate is good but coarse enough so that the population in each bin is sufficient to obtain good statistics. For most runs, step sizes of $0.05 \mathrm{~mm}$ in $x$ and $y$ and $1 \mu \mathrm{rad}$ in $x^{\prime}$ constitute a good compromise.

Figure 5.1,(a) shows the incoming beam distribution as a function of the horizontal position, altered by the periodic pattern mentioned earlier. The beam horizontal dimension being about six times larger than the crystal width, the density of particles is almost constant with respect to $x$. It is also the case for the fraction of channeled particles suggesting that the crystal performance is homogeneous along that dimension. Figure 5.1,(b) shows the distribution as a function of the horizontal angle. There is an offset between the angle of maximum channeling rate and the average beam orientation (2.5 $\mu \mathrm{rad}$ in this case). This offset, emphasized by the arrows in fig. 5.1,(b), indicates that the crystal was not positioned exactly in its ideal orientation. In the simulation, the crystal orientation is constant and it is rather the incoming beam which is rotated towards a mean angle equal to the offset value. In the graph (c) pertaining to the vertical hit position, channeling is found to be favored on the upper side because of torsion changing the channel orientation with $y$. The vertical coordinate of maximum channeling is the one where torsion implies a change in orientation compensating the horizontal angle offset seen in (b). Finally, from (d), one sees that the channeled fraction remains constant for varying vertical angles, reason for which the vertical angle will not be considered in the following analysis.

\subsection{3 | Torsion measurement}

Results concerning the intrinsic channeling performance of a given crystal must be expressed independently of any torsion. Its influence on the data can be compensated by adjusting the incoming angle of a track as a function of its vertical hit position. The 


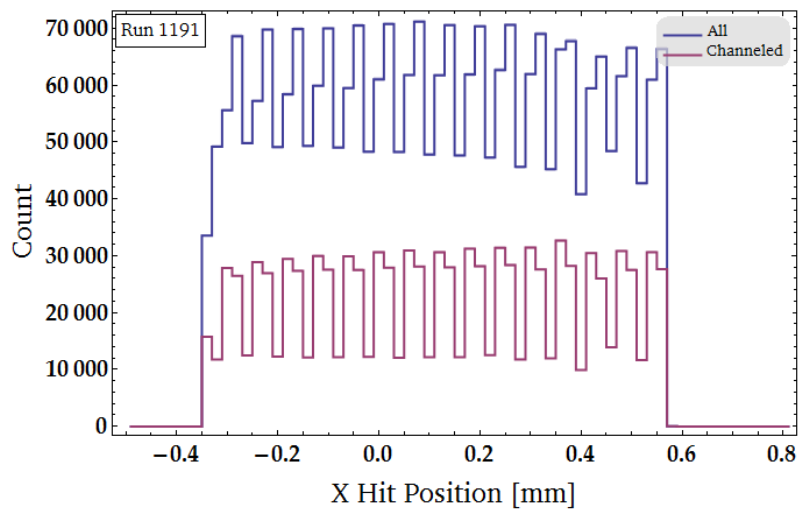

(a)

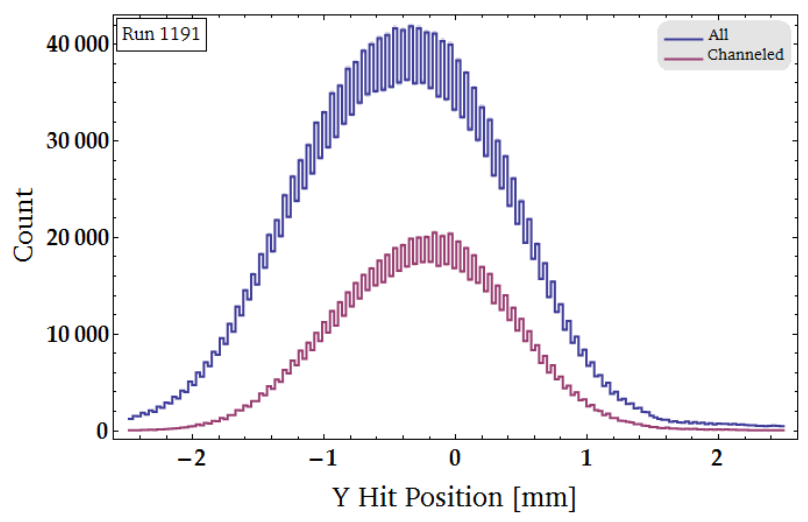

(c)

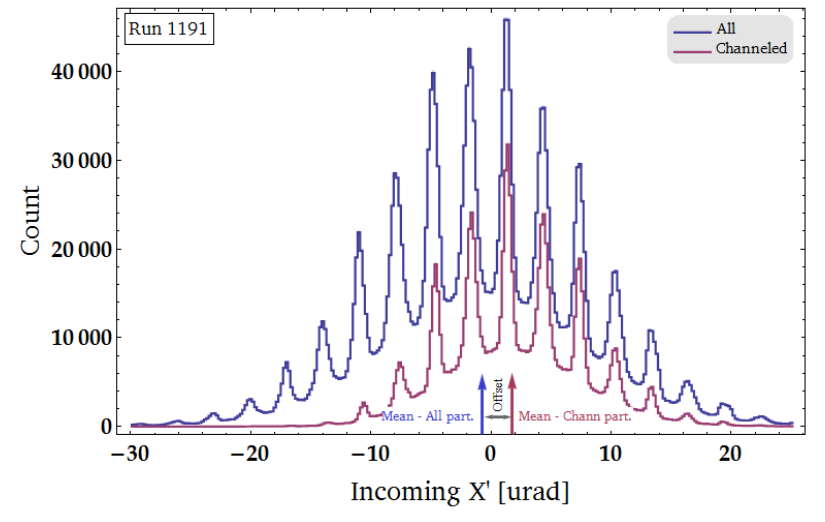

(b)

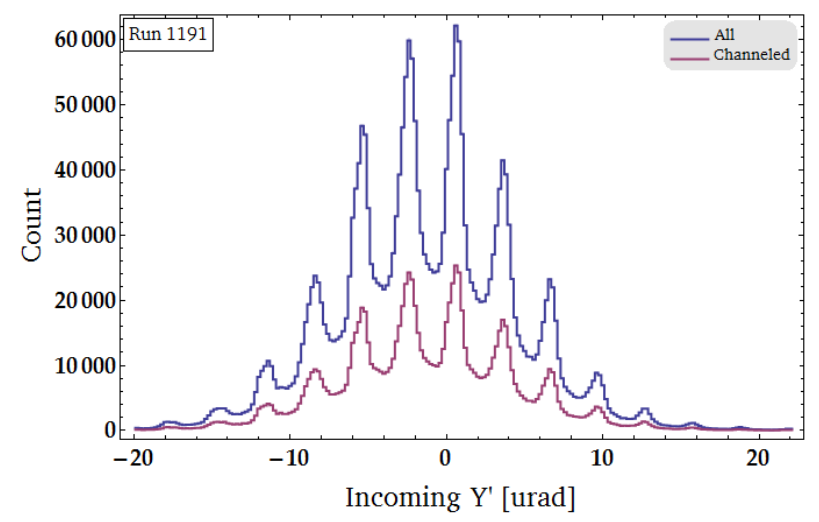

(d)

Fig. 5.1. Incoming beam distributions in run 1191 as a function of : (a) the horizontal hit position, (b) the horizontal incoming angle, (c) the vertical hit position and (d) the vertical incoming angle. Blue curves relate to all particles and red curves to channeled ones. A pattern with a period $30 \mu \mathrm{m}$ in $x$ and $y$ and $30 \mu \mathrm{rad}$ in $x^{\prime}$ and $y^{\prime}$ is deforming the distributions. The linear interpolation on which the track reconstruction is based does not take into account the non-linear aspects of the charge deposition in the detector, favoring in fact locations close to the strip implants. 


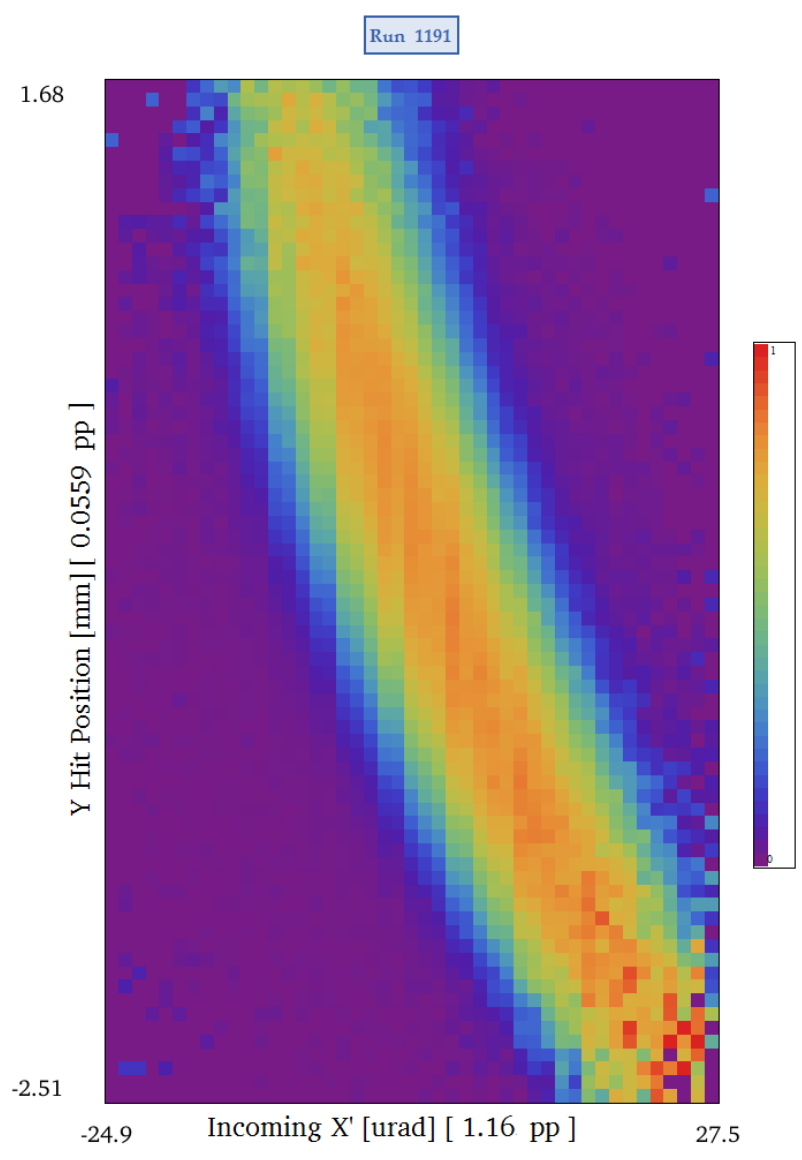

(a)

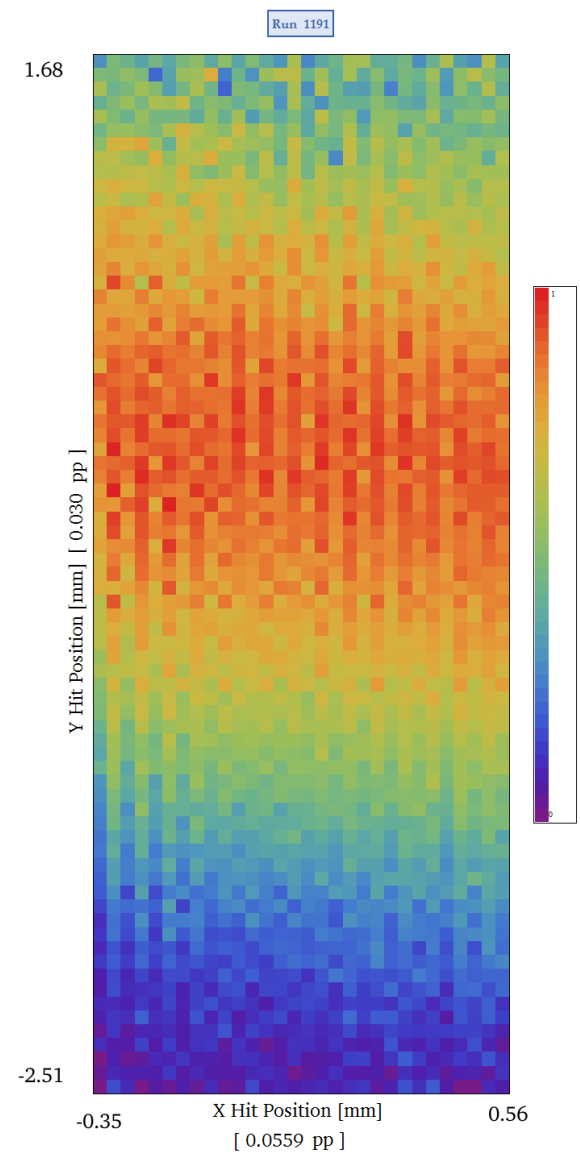

(b)

Fig. 5.2. Two-dimensional array plots giving the channeling rate (colored scale) as a function (a) of $y$ and $x^{\prime}$, putting in evidence the effect of crystal torsion; (b) of $x$ and $y$, showing that the channeling rate is virtually independent of $x$. The sizes of the bins are indicated on the corresponding axes.

torsion $T$, not known beforehand, can be measured using the 3D binning mentioned earlier. In each bin, the number of channeled particles ${ }^{3}$ divided by the total number of events is the channeling rate for that specific bin. Fig. 5.2,(a) shows the rate in each bin as a function of the vertical position and horizontal angle, the population being integrated over the third dimension, the horizontal position $x$. Torsion is clearly visible as inducing a channel orientation dependence on $y$. For each bin along $y$, the channeling rate follows a bell curve along $x^{\prime}$. The mean of this distribution is located at the ideal horizontal orientation $x_{\mathrm{opt}}^{\prime}(y)$ corresponding to that $y$ coordinate. Torsion is the angular coefficient of the linear fit of the couples $\left(x_{\mathrm{opt}}^{\prime}(y), y\right)$. Here, it is equal to $-7.65 \pm 0.14 \mu \mathrm{rad} / \mathrm{mm}$. Fig. 5.2,(b) shows the channeling rate as well, this time as a function of the horizontal and vertical position, events being integrated on $x^{\prime}$. One can see that crystal performance

\footnotetext{
${ }^{3}$ Still defined as particles within 3 standard deviations from the average channeling kick.
} 


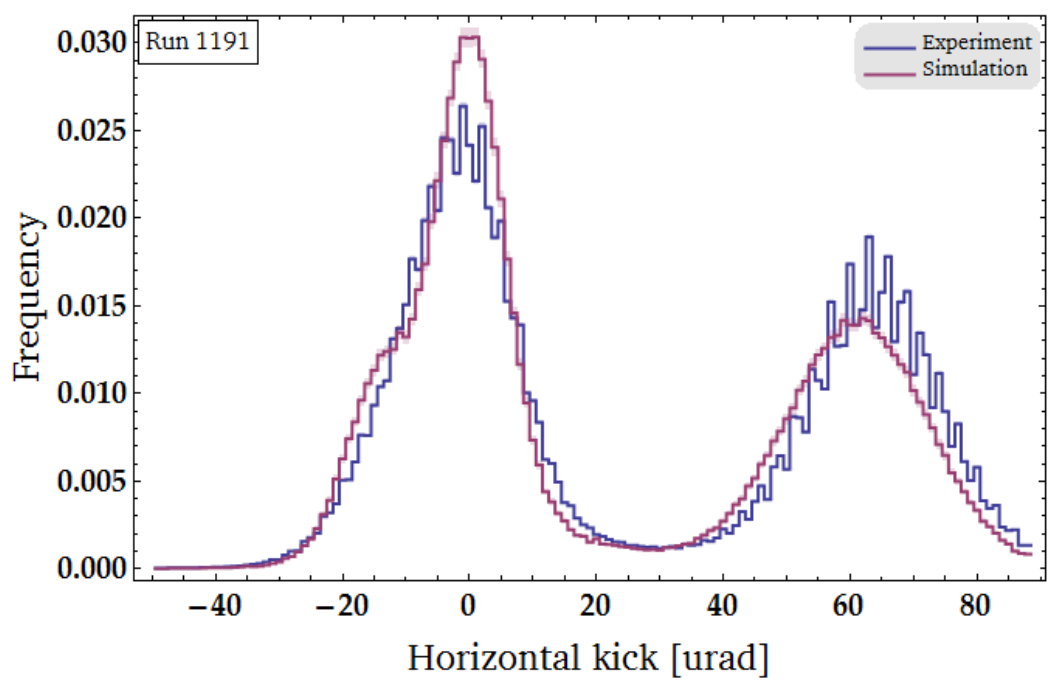

Fig. 5.3. Angular deflection distribution for run 1191. The experimental data is shown in blue and the simulation in red.

is homogeneous in $x$. In $y$ it is not the case as there is some torsion and the crystal ideal orientation is gradually shifted when going towards the top or bottom of the crystal. This causes a decrease of the channeling rate as the majority of incoming particles, having $\theta_{x}$ distributed close to a Gaussian distribution (centered in fig. 5.2), will nto see the crystal along its channels orientation. This effect is stronger in the lower part of the crystal as the ideal orientation shifts further away from the beam mean orientation there than in the upper part.

At this stage of the analysis, all parameters needed for the simulation are known :

- Horizontal angular offset (as the crystal was not perfectly aligned with the beam).

- The vertical position of the beam.

- The angular divergence in both planes (especially the horizontal one).

- The torsion coefficient.

\subsection{4 | Comparison with simulation}

Comparing simulation with experiment in a crystal channeling framework consists in evaluating quantities like the channeling rate, the angular spread at channeling exit, the dechanneling rate and the angular spread for VR/AM particles. They are obtained from the (horizontal) angular deflection distribution. However, one cannot draw conclusions about the crystal performance from this distribution yet as it includes all particles enter- 
ing the crystal even with large angles, thus having a low probability of getting channeled independently of the crystal quality.

Table 5.2. Key figures of the angular deflection distribution for the experimental run 1191 and the corresponding simulation. The rate is expressed in $\%$ and the deflection characteristics in $\mu$ rad.

\begin{tabular}{lcc}
\hline RUN 1191 & EXPERIMENT & SIMULATION \\
\hline Number of events & 2619108 & 400000 \\
CH rate & $39.58(6)$ & $37.03(15)$ \\
CH peak st. dev. & $8.81(1)$ & $10.90(4)$ \\
DC rate & $3.79(3)$ & $2.80(5)$ \\
VR/AM peak mean & $-7.01(2)$ & $-3.32(4)$ \\
VR/AM peak st. dev. & $9.30(1)$ & $9.46(3)$ \\
\hline
\end{tabular}

The distributions of horizontal deflections at crystal exit are shown in fig. 5.3 for the experiment in blue and the simulation in red. Related figures are compiled in table 5.2. The agreement is quite good for the two peaks and the dechanneling profile.

A small discontinuity in the VR/AM peak slope can be seen around $-8 \mu \mathrm{rad}$. It corresponds to the transition between AM and VR regimes. Although it is smoothed by the introduction of the hybrid mode (to be seen in p. 36) it is still visible as the nominal VR kick is larger than the MCS spread.

\subsection{5 | Torsion compensation}

In order to know the efficiency of a crystal, the data must be filtered down to keep only angles $\theta_{x} \ll \theta_{c}$ in addition to the torsion compensation. The latter consists in a modification of the incoming angle of the track taking into account the actual channel orientation at the $y$ position of impact :

$$
x^{\prime} \rightarrow x^{\prime}-T \Delta y
$$

where $\Delta y$ is the vertical offset with respect to the point of maximal channeling before compensation. The beam distribution after this manipulation is shown in fig. 5.4 where the blue curve stands for all particles and the red curve for the channeled ones. Channeled particles are now more localized around the ideal orientation of the crystal where the proportion of channeled particles has increased.

Fig. 5.5 shows the channeling rate in percent along each of the three investigated dimensions for incoming angles allowing channeling in fig. $5.4([-12,12] \mu \mathrm{rad})$. From the top graph, it can be seen that the channeling rate is constant along $x$, as deduced from 


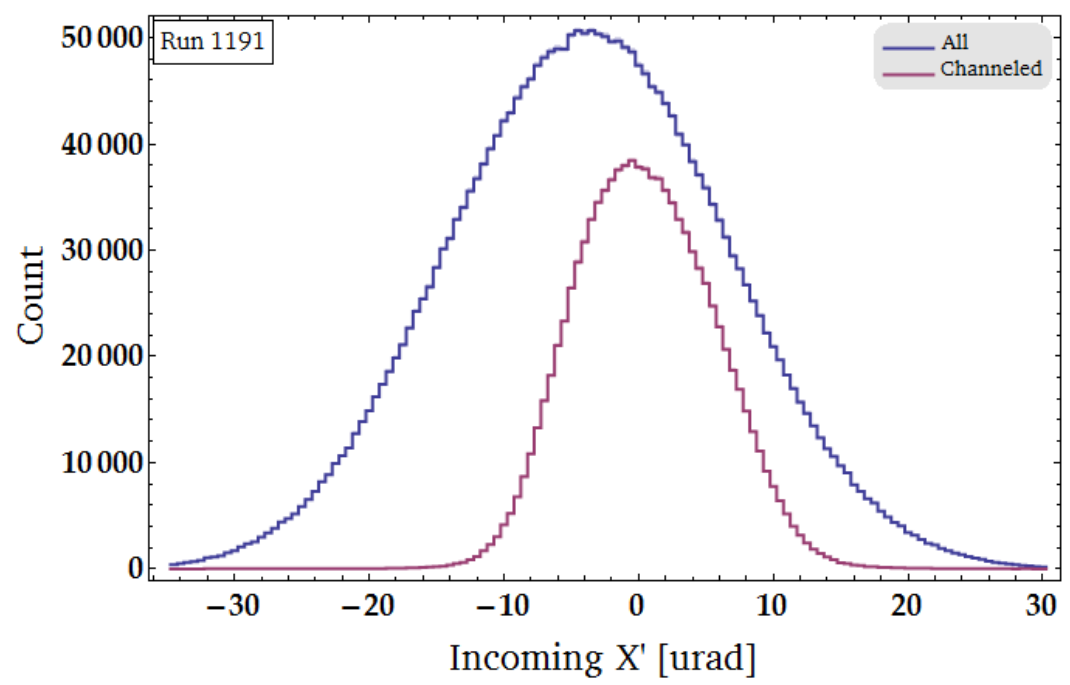

Fig. 5.4. Distribution of the incoming angles after torsion compensation for run 1191. The blue curve represents the entire population and the red curve, the channeled particles.

fig. 5.2,(b). For what concerns $y$, the channeling rate is low in the bottom of the crystal and then increases up to a plateau which is eventually slightly decreasing. This is due to the linear fitting failing to compensate exactly the torsion appearing in fig. 5.2,(a), clearly not linear. In the lower part, where the fit is the least accurate, the rate estimation drops strongly. Finally, along $x^{\prime}$, the curve just reflects the fact that the closer the incoming angle is to the perfect orientation, the larger the probability of getting channeled. The efficiency, corresponding to very small incoming angles, is found to be equal to $81 \%$.

Selecting incoming particles only in a very small angular window ( $\pm 1 \mu \mathrm{rad})$, as in fig. 5.6, allows to obtain the exit kick distribution for an ideal perfectly aligned beam without divergence. The resulting large channeling peak reflects the high channeling efficiency of the considered crystal. 

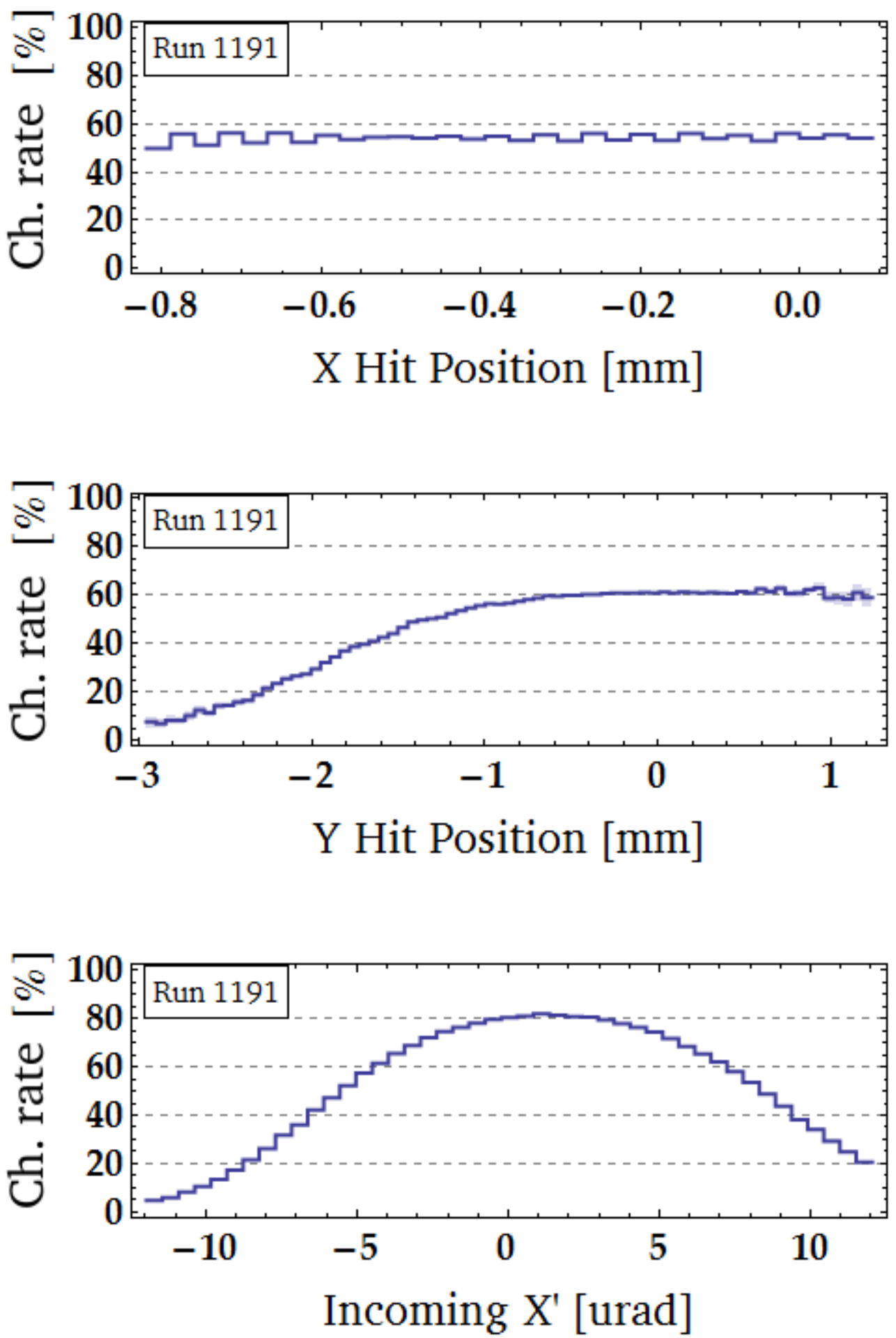

Fig. 5.5. Channeling rate along $x, y$ and $x^{\prime}$ obtained for run 1191 after torsion compensation. 95\% confidence intervals are indicated in light blue. 


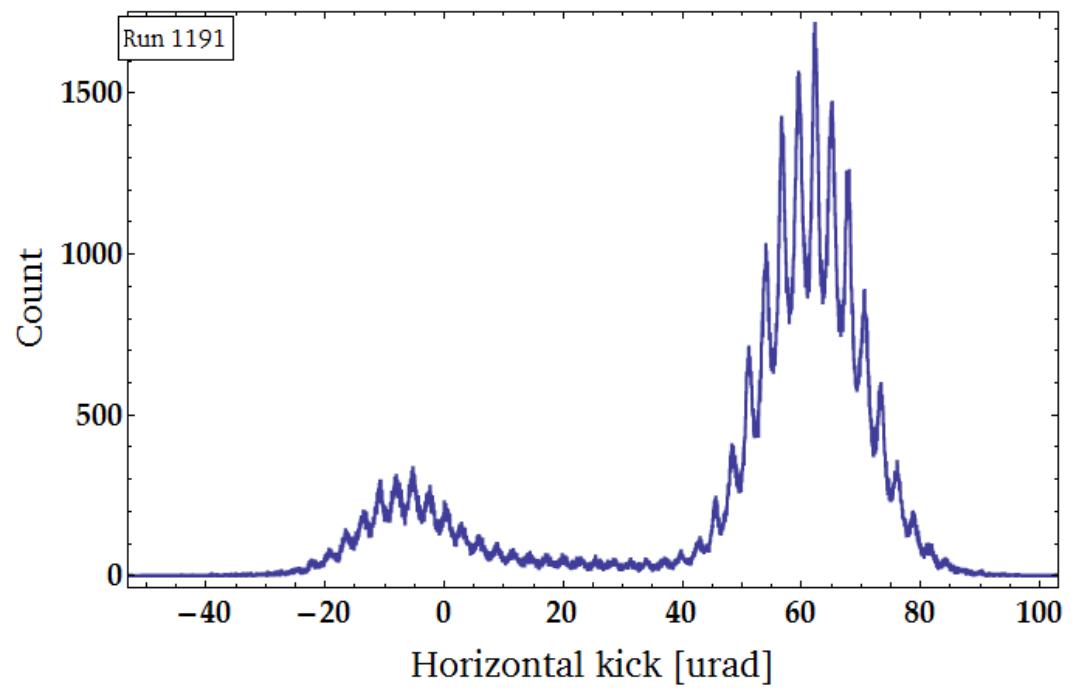

Fig. 5.6. Exit kick distribution after torsion compensation and narrow filter of the incoming angle $( \pm 1 \mu \mathrm{rad}$ ) for the LHC strip crystal. The channeling rate is maximum in this case. Artifacts due to the non-linearity of the charge deposition in the strip detectors are visible. 


\subsection{Strip crystal for the SPS (STF 50)}

The crystal STF 50 is a $2 \mathrm{~mm}$-long strip crystal with a large bending angle (170 $\mu \mathrm{rad}$ ) manufactured in INFN Ferrara. It has been tested in runs 889 to 892 in July 2012 in the H8 beamline and is a candidate for installation as part of the UA9-SPS experimental setup. The critical angle for $400 \mathrm{GeV} / \mathrm{c}$ protons in this crystal is $6.6 \mu \mathrm{rad}$.

\subsection{1 | Torsion measurement}

After retaining only crystal-hitting tracks and classifying them in a suitable binning along the $x, x^{\prime}$ and $y$ dimensions, the channeling rate map as a function of $x^{\prime}$ and $y$ is obtained (see fig. 5.7). The torsion value found through the same fitting process as in sec. 5.2 .3 is $4 \mu \mathrm{rad} / \mathrm{mm}$.

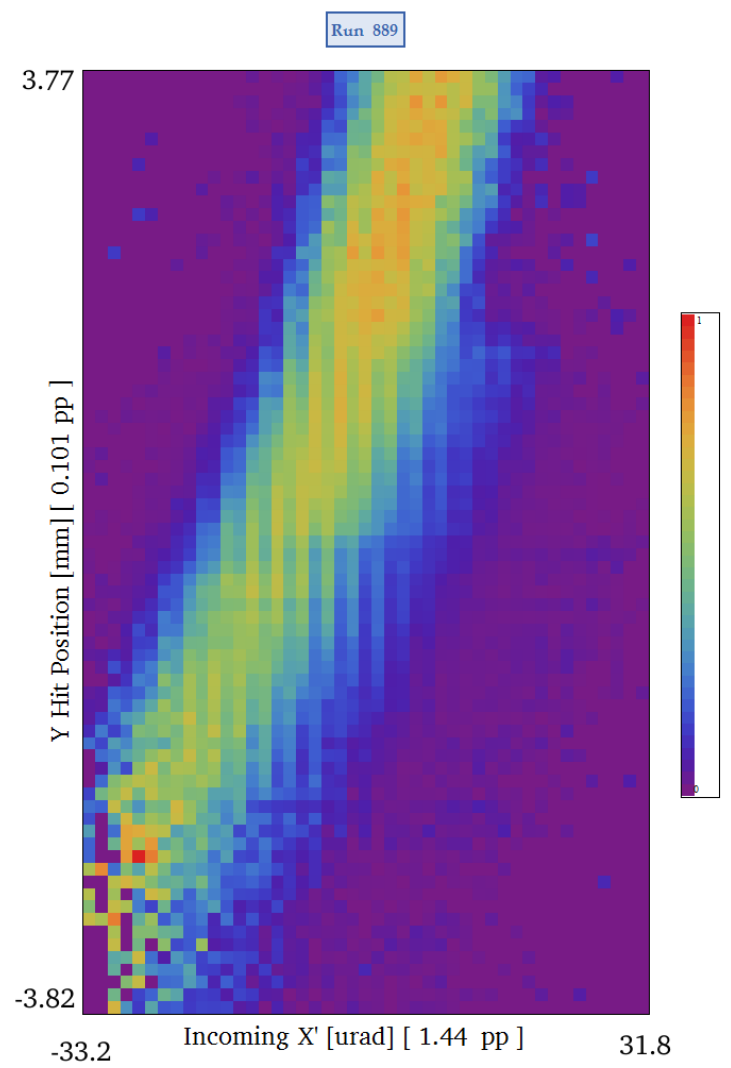

Fig. 5.7. Channeling rate map of the STF 50 crystal as a function of the horizontal incoming angle and the vertical hit position. The effect of torsion is clearly visible (cf. fig. 5.2). 


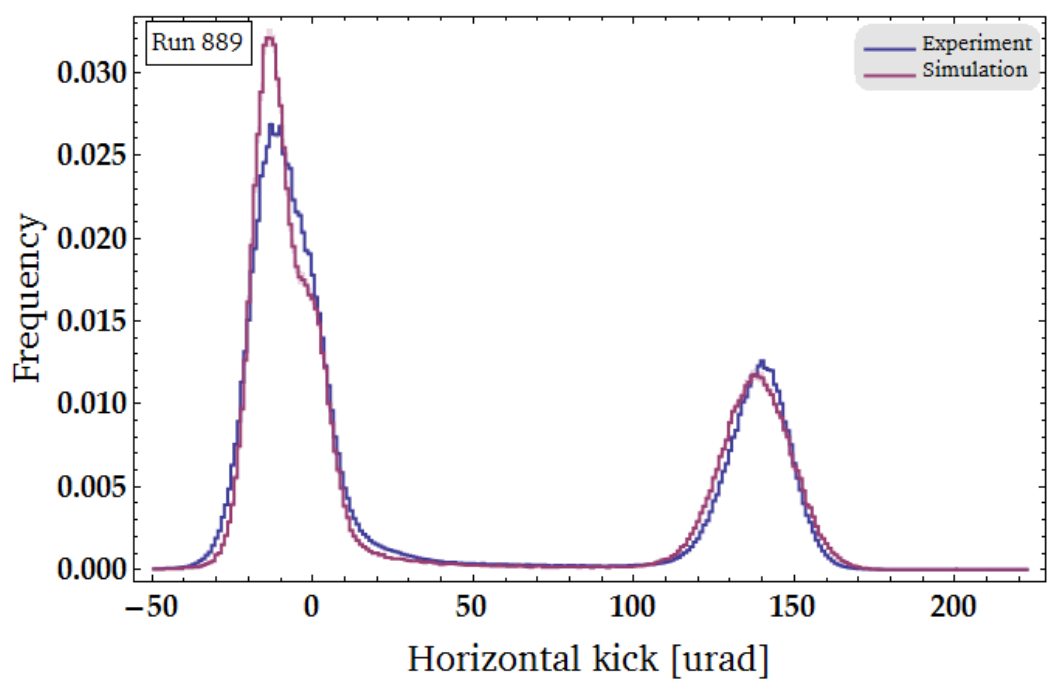

Fig. 5.8. Angular deflection distribution for crystal STF 50 - run 889. The experimental data is shown in blue and the simulation in red.

Table 5.3. Key figures of the angular deflection distribution for the experimental run 889 and the corresponding simulation. The rate is expressed in $\%$ and the deflection characteristics in $\mu \mathrm{rad}$.

\begin{tabular}{lcc}
\hline RUN 889 & EXPERIMENT & SIMULATION \\
\hline Number of events & 2489137 & 400000 \\
CH rate & $29.1(6)$ & $31.5(2)$ \\
CH peak st. dev. & $9.26(2)$ & $10.63(4)$ \\
DC rate & $3.65(3)$ & $2.67(5)$ \\
VR/AM peak mean & $-8.23(2)$ & $-9.37(4)$ \\
VR/AM peak st. dev. & $10.15(1)$ & $9.32(3)$ \\
\hline
\end{tabular}

\subsection{2 | Exit kick distribution}

The exit kick distribution for the STF 50 crystal is shown in fig. 5.8 for the experiment (blue) and the simulation (red). Respective figures can be found in table 5.3. The agreement is very good in most respects. Analogously to the previous case, the VR/AM peak appears slightly higher and narrower in the simulation.

\subsection{3 | Channeling rates}

Figure 5.9 shows the channeling rate curves after torsion compensation. The rate is constant along the $x$ dimension as for the previous case (apart from the edges). On 

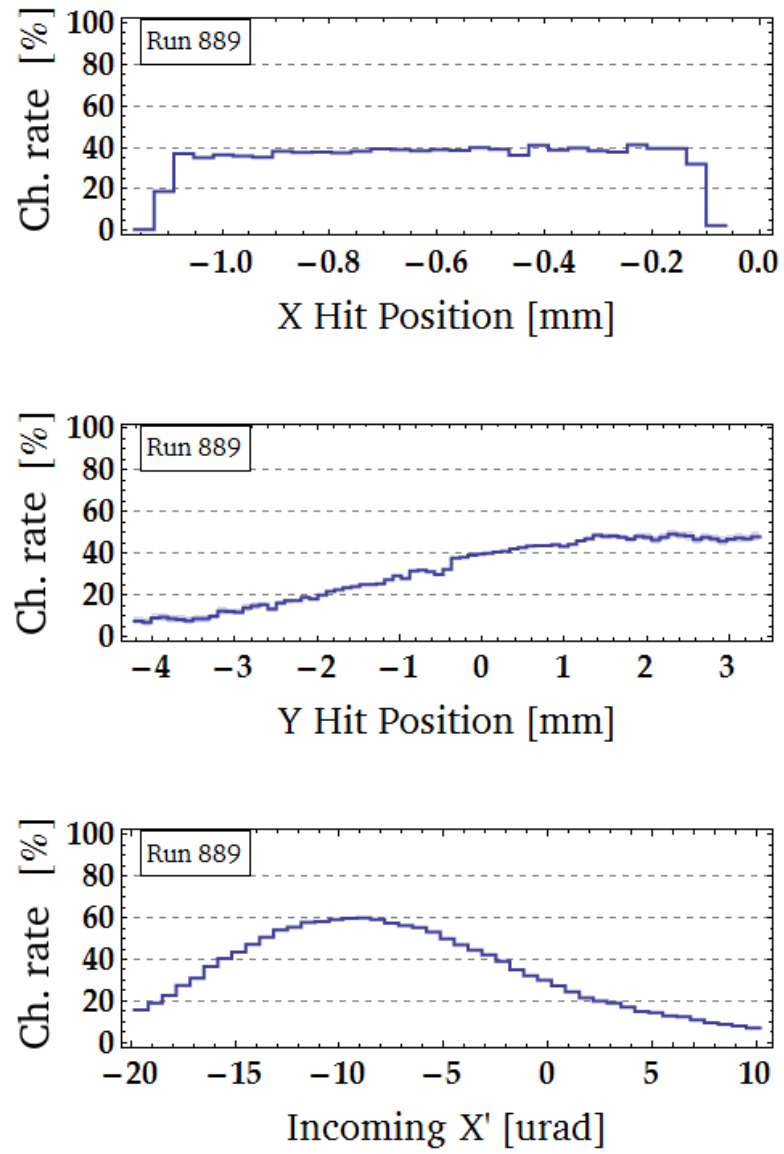

Fig. 5.9. Channeling rate along $x, y$ and $x^{\prime}$ obtained for run 889 after torsion compensation.

the contrary, along the vertical dimension, channeling is more frequent towards the top of the crystal. The last graph indicates a relative misorientation of $10 \mu \mathrm{rad}$. Here the efficiency approaches $60 \%$.

\subsection{4 | Volume reflection orientation}

The volume reflection orientation is reached by rotating the crystal by an angle between $\theta_{c}$ and $\theta_{b}$ in the direction opposite to its bend with respect to the ideal channeling orientation. In general, to minimize interferences from the channeling or amorphous regimes, the crystal is positioned at an angle of about $\theta_{b} / 2$ off the ideal orientation. In this orientation, the resulting kick distribution at exit is shown (in a logarithmic scale to better appreciate volume capture) in fig. 5.10 for the experiment (blue) and the simulation (red). In this case, the main peak corresponds to reflected particles only and its mean value is the kick given through the volume reflection process. Its spread is related 


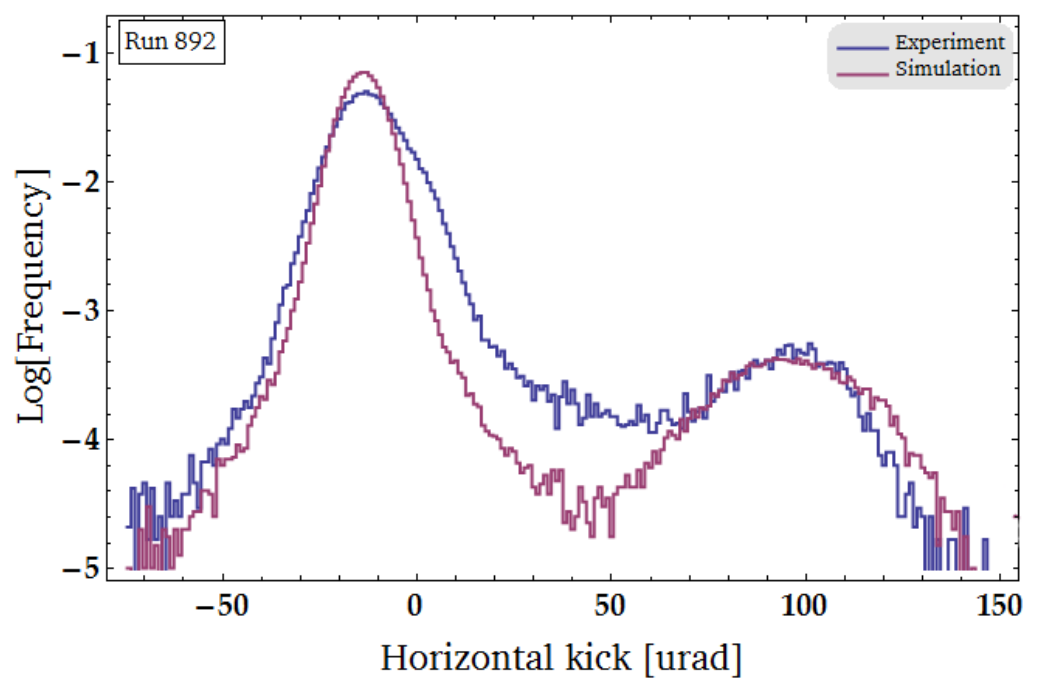

Fig. 5.10. Angular deflection distributions from the experiment (blue) and the simulation (red) for the STF 50 crystal in VR orientation. The main peak corresponds to reflected particles. The bump at about $100 \mu \mathrm{rad}$ is due to particles being captured and staying channeled until the end. Particles being dechanneled after the capture are distributed in between.

Table 5.4. Key figures of the angular deflection distribution for the run in volume reflection. The rate is expressed in \% and the deflection characteristics in $\mu \mathrm{rad}$.

\begin{tabular}{l|cc}
\hline & Exp. & Sim. \\
\hline VR Peak mean & $-12.95(4)$ & $-14.66(2)$ \\
VR Peak rms & $8.36(2)$ & $5.81(1)$ \\
VC rate & $4.38(8)$ & $3.10(5)$ \\
\hline
\end{tabular}

to the MCS over the length of the crystal.

The volume capture peak, seen at an angle close to $100 \mu \mathrm{rad}$ appears rather well reproduced by the simulation. The calculated volume capture rate is in relatively good agreement with the experiment. The captured particles can be dechanneled and populate the region between the VR and VC peaks. The dechanneling of captured particles is much rarer in the simulation than what the experiment shows. According to these findings, one might think of reevaluating the way the initial transverse energy of captured particles is assigned. Finally, one can notice an asymmetry in the VR peak, with a larger population on its positive side. In the simulation, the peak is purely constituted of reflected particles. In reality, some particles in amorphous mode with a deflection around 0 caused by MCS might subsist in this orientation which would explain this shape. 


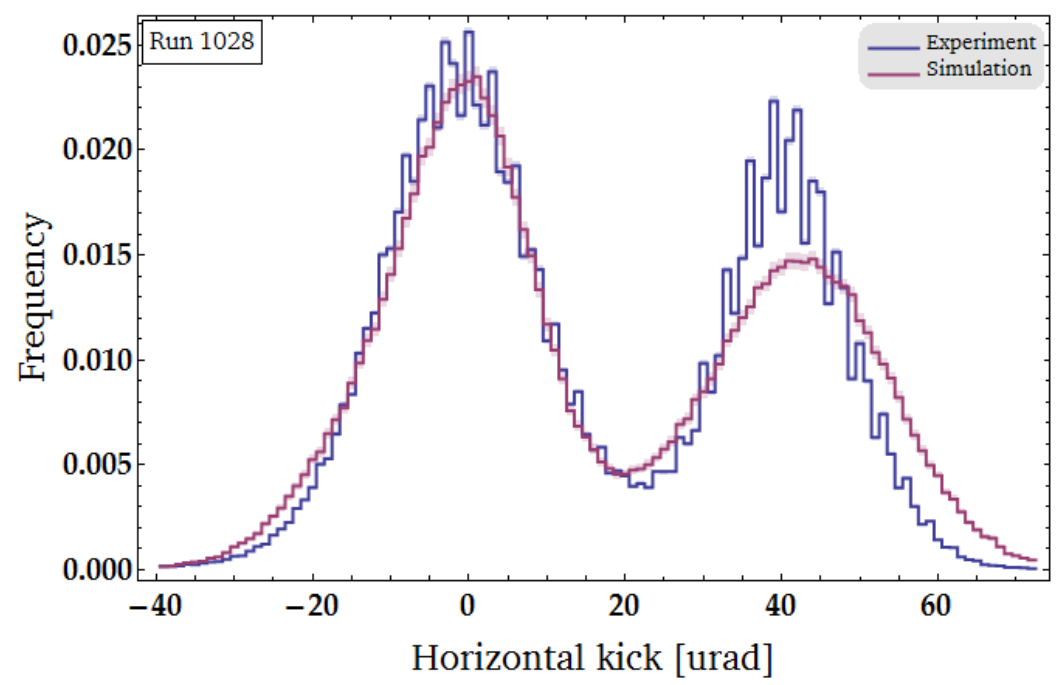

Fig. 5.11. Angular deflection distributions from the experiment (blue) and the simulation (red) for the QMP 28 crystal.

\subsection{Quasi-mosaic crystal for the LHC (QMP 28)}

Quasi-mosaic crystals differ from strip crystals by the type of mechanical curvature used to deflect particles (see chapter 4). Quasi-mosaic crystals are also wider and their curvature is slightly varying in orientation along their transverse dimensions. Instead of treating all particles impacting the crystal, the analysis of a QM crystal run focuses on a zone within few millimeters from the edge where channeling is favored by the alignment procedure.

\subsection{1 | Exit kick distribution}

The torsion is calculated via the method described above, yielding a value of $1.7 \mu \mathrm{rad}$. The corresponding angular deflection distributions are shown in fig. 5.11. The agreement between simulation and data is quite good, in particular for the VR/AM peak and the dechanneling zone. The MCS makes the dechanneling rate harder to evaluate precisely, as the edges of the two peaks overlap. For a beam at nominal LHC energy though, MCS will be strongly reduced and this profile would change substantially. 

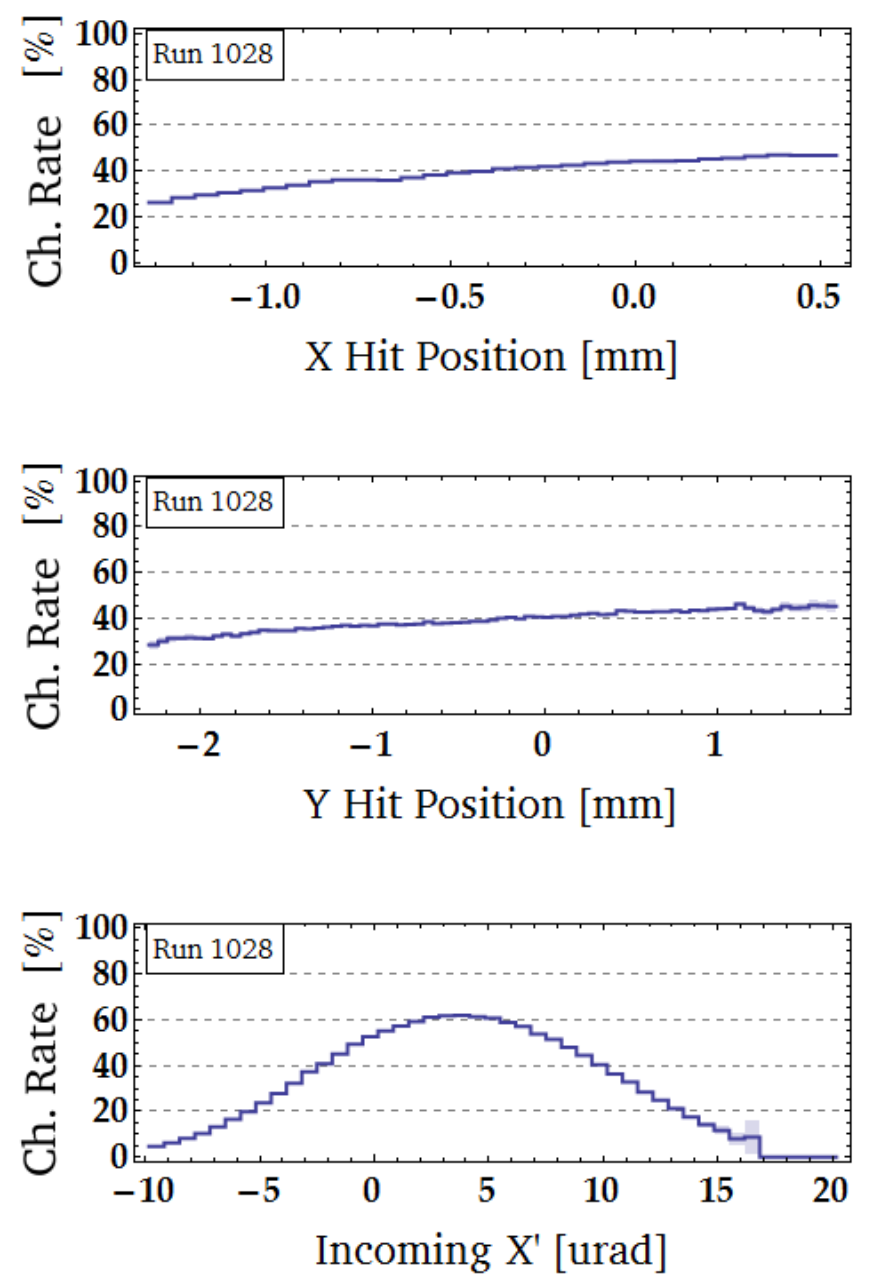

Fig. 5.12. Channeling efficiency along $x, y$ and $x^{\prime}$ obtained for the QMP 28 crystal after torsion compensation.

\subsection{2 | Channeling rates}

After torsion compensation, the channeling rate of the QM crystal is evaluated along the three binning axes and results are shown in fig. 5.12. The rate becomes larger with increasing values of $x$ because the QM crystal has an anticlastic curvature that makes the channels orientation change with $x$. Channels in the part of the crystal closer to the holder turn out to be better aligned with the beam. The variation in $y$ is related to the QM crystal primary curvature inducing a variation of the orientation along $y$. Finally, the bottom graph gives the maximum efficiency attained (62\%). 
Table 5.5. Key figures of the angular deflection distribution for the experimental run 1028 and the corresponding simulation. The rates are expressed in $\%$ and the deflection characteristics in $\mu$ rad.

\begin{tabular}{lcc}
\hline RUN 1028 & EXPERIMENT & SIMULATION \\
\hline Number of events & 2313383 & 400000 \\
CH rate & $38.9(1)$ & $35.4(2)$ \\
CH peak st. dev. & $7.01(1)$ & $9.79(4)$ \\
DC rate & $9.96(4)$ & $5.34(7)$ \\
VR/AM peak mean & $-4.16(2)$ & $-1.03(5)$ \\
VR/AM peak st. dev. & $8.63(1)$ & $11.7(1)$ \\
\hline
\end{tabular}


Table 5.6. Crystal parameters as determined through data analysis. The channeling efficiency is very sensible to the filtering. Here an angular window of $1 \mu \mathrm{rad}$ is typically applied.

\begin{tabular}{lcccc}
\hline CRYstAL & RUN & $\begin{array}{c}\text { BENDING ANGLE } \\
{[\mu \mathrm{rad}]}\end{array}$ & $\begin{array}{c}\text { TORSION } \\
{[\mu \mathrm{rad} / \mathrm{mm}]}\end{array}$ & $\begin{array}{c}\text { MAX. EFFIC. } \\
{[\%]}\end{array}$ \\
\hline STF 47 & 608 & $35.5(1)$ & $3.0(7)$ & 57 \\
STF 48 & 630 & $141.8(6)$ & $7.8(5)$ & 59 \\
STF 50 & 889,892 & $138.5(1)$ & $4.0(2)$ & 60 \\
STF 51 & 899 & $36.6(1)$ & $0.81(20)$ & 45 \\
STF 70 & 1240 & $54.50(15)$ & $0.35(12)$ & 82 \\
STF 71 & 1191 & $61.26(2)$ & $7.7(2)$ & 81 \\
QMP 26 & 1012 & $50.83(2)$ & $9.8(1.1)$ & 58 \\
QMP 28 & 1028 & $41.20(15)$ & $1.7(1)$ & 62 \\
\hline
\end{tabular}

\subsection{Summary of additional benchmarking cases}

In this section, additional experimental runs are briefly examined before results are summarized and discussed more extensively.

During the last 3 years, more than 20 crystals have been tested in $\mathrm{H} 8$ including multistrips, crystals with variable curvature and thin flat crystals. Ultimately, this study could be extended to crystals with a variable curvature quite easily ${ }^{4}$. The case of multi-strip crystals is even simpler, as their characteristics are naturally reproduced with the use of FLUKA geometry.

Concentrating on the strip and quasi-mosaic devices, table 5.6 gives a list of other crystals that have been tested. Their corresponding run number is indicated along with the nominal bending and torsion, as measured during the experiment and the channeling efficiency they allowed. It has to be noted that the crystal torsion can be modified via the holder and is not an intrinsic characteristic of the crystal. The graphs containing the angular deflection distributions of these additional runs can be found in appendix $\mathrm{C}$.

Table 5.7 lists the means and standard deviations of the channeling peaks and the VR/AM peaks. Minor differences are seen among which the fact that the simulated channeling peaks have a slightly larger standard deviation in almost all the runs. Additionally, the simulated AM/VR peaks show few $\mu$ rad offsets probably due to the limited applicability of the geometrical model giving the VR kick value (eq 2.34). In the case of LHC crystals, the channeling peak can be poorly defined (see fig. C.3, p. C.3 for run 899).

\footnotetext{
${ }^{4}$ The radius is in fact already reevaluated at each step in the current model to take into account the weak effect of torsion on the radius which depends on the vertical component of the particle motion.
} 
Table 5.7. Channeling and reflected/amorphous peak characteristics from experiment and simulation. Values are given in $\mu \mathrm{rad}$.

\begin{tabular}{c|cccc|cccc}
\hline \multirow{2}{*}{ RUN } & \multicolumn{3}{|c|}{ CHANNELING PEAK } & \multicolumn{4}{c}{ VR/AM PEAK } \\
& \multicolumn{2}{|c}{ Experiment } & \multicolumn{2}{c}{ Simulation } & \multicolumn{2}{c}{ Experiment } & Simulation \\
& Mean & Sigma & Mean & Sigma & Mean & Sigma & Mean & Sigma \\
\hline 608 & $35.53(3)$ & $7.27(2)$ & $34.83(6)$ & $10.17(4)$ & $-1.06(3)$ & $10.7(1)$ & $-2.28(5)$ & $13.58(4)$ \\
630 & $141.8(1)$ & $11.3(1)$ & $142.0(1)$ & $10.68(4)$ & $-1.96(3)$ & $8.40(2)$ & $-3.75(3)$ & $8.09(2)$ \\
889 & $138.5(1)$ & $9.26(2)$ & $137.7(1)$ & $10.63(4)$ & $-8.23(2)$ & $10.2(1)$ & $-9.37(4)$ & $9.32(3)$ \\
899 & $36.64(1)$ & $7.38(1)$ & $34.86(4)$ & $9.97(4)$ & $-5.15(1)$ & $9.01(1)$ & $-6.12(4)$ & $10.7(1)$ \\
1012 & $50.83(2)$ & $9.14(2)$ & $50.82(6)$ & $10.98(4)$ & $-4.21(2)$ & $9.00(1)$ & $-3.18(4)$ & $9.32(3)$ \\
1028 & $41.20(2)$ & $7.01(1)$ & $41.84(5)$ & $9.79(4)$ & $-4.16(2)$ & $8.63(1)$ & $-1.03(5)$ & $11.7(1)$ \\
1191 & $61.26(2)$ & $8.81(1)$ & $60.18(6)$ & $10.90(4)$ & $-6.39(2)$ & $9.97(1)$ & $-3.32(4)$ & $9.46(3)$ \\
1240 & $54.50(2)$ & $8.55(1)$ & $54.05(5)$ & $10.56(3)$ & $-5.61(2)$ & $9.09(1)$ & $-6.37(5)$ & $10.6(1)$ \\
\hline
\end{tabular}

Then, channeled, dechanneled and amorphous-mode particle angular distributions overlap significantly, leading to a difference of up to $7 \%$ between experiment and simulation whereas the distribution profile is shown to be consistent nonetheless. In addition, QM crystals have a non-uniform curvature profile, the variations of which are not reproduced by the simulation leading to an overestimation of the channeling rate.

Looking at table 5.8 one sees that the channeling rate, which is the most critical quantity in view of the design of a collimation layout, is reasonably well reproduced. In two cases, the overestimation reaches $7 \%$ of the impacting beam population. The dechanneling rate tends to be underestimated by the simulation. In some cases, the experimental values are large numbers that can result from a too small separation between the two peaks in comparison with the MCS rms deflection at that energy. The dechanneling population is then identified with difficulty.

Finally, one must not forget that there is a non-negligible measurement error due to the non ideal apparatus : the error reaches $5.4 \mu \mathrm{rad}$ in the horizontal angle dimension. In addition, the benchmarking of the code must always be put in the perspective of the quality of the crystals. The model presupposes that crystals are ideal and does not take into account any defect of the crystalline lattice. 
Table 5.8. Channeling and dechanneling rates, expressed in \%, obtained through data analysis for experimental runs and the corresponding simulations. Populations are defined iteratively as comprised within 3 standard deviations from angular kicks given in table 5.7 (see sec. 3.7.1).

\begin{tabular}{c|cccc}
\hline \multirow{2}{*}{ RUN } & \multicolumn{3}{|c}{ Channeling Rate } & \multicolumn{3}{c}{ DeChanNeling RATE } \\
& Exp. & Sim. & Exp. & Sim. \\
\hline 608 & $29.04(15)$ & $29.15(14)$ & $3.31(6)$ & $2.29(5)$ \\
630 & $25.12(10)$ & $28.16(14)$ & $8.05(7)$ & $2.06(4)$ \\
889 & $29.04(6)$ & $31.35(15)$ & $3.65(2)$ & $2.67(5)$ \\
899 & $24.99(3)$ & $31.41(15)$ & $14.7(1)$ & $9.20(9)$ \\
1012 & $32.54(6)$ & $37.06(15)$ & $4.54(28)$ & $2.27(5)$ \\
1028 & $36.87(6)$ & $35.44(15)$ & $9.96(39)$ & $5.34(7)$ \\
1191 & $39.58(6)$ & $37.03(15)$ & $2.78(20)$ & $2.80(5)$ \\
1240 & $50.73(3)$ & $47.86(16)$ & $3.49(22)$ & $3.98(6)$ \\
\hline
\end{tabular}





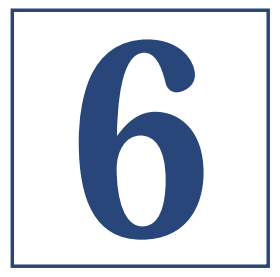

\section{SIXTH CHAPTER}

\section{CONCLUSIONS AND PERSPECTIVES}

A new event generator has been presented, simulating the coherent physical processes related to crystal channeling. This new tool has been developed in order to be fully incorporated in FLUKA, the multipurpose Monte Carlo code used at CERN for beammachine interaction calculations. It allows comprehensive treatment of beam particles and secondaries and is designed to be able to cope with positively charged hadrons at any (high) energy in any synchrotron crystal-assisted collimation layout. Until now, state-of-the-art channeling codes have not yet been interfaced with beam tracking codes like SixTrack that are commonly used in the design of large accelerators, the CPU time implied by the equation of motion integration being a severe issue. On the other hand, an empirical channeling routine has already been coupled to SixTrack. However as it is based on data fit at SPS energy, an extrapolation to the LHC conditions is needed. Therefore, the implementation of channeling in FLUKA, whose interaction models are physics-driven and well benchmarked, represents an interesting step especially in the perspective of the SixTrack-FLUKA coupling presently under development at CERN.

In the first part of this thesis, it is has been reminded how crystals form channels along specific directions. Crystal channeling applications concern almost exclusively diamond-shaped lattices, such as Silicon or Germanium ones. In these structures, string or planes of atoms induce axial or planar channeling respectively. Such crystals can be bent in order to be able to deviate beam particles. In particular, they can extract the beam halo onto downstream collimators. The smaller size of the crystal compared with a standard primary LHC collimator ( $~ 300$ times smaller) and the reduction of the interaction rates for channeled particles can lead to a major reduction of inelastic events which are responsible for losses in sensitive regions of the machine. 
This work has been focused on planar channeling because of its wide use in crystal collimation nowadays. The electromagnetic potential due to adjacent atomic planes in a well-oriented lattice is approximated by the continuous potential model incorporating the atomic thermal motion and the Moliere screening function with the addition of the centrifugal potential due to crystal bending. Particles having an energy associated to the transverse dimension $E_{x}$ lower than the effective barrier $U_{0}$, depending on crystal parameters such as bending radius and torsion, are channeled. Particles entering channeling mode undergo interactions modifying $E_{x}$. If it goes above $U_{0}$, they get dechanneled and propagate in amorphous mode in the remaining of the crystal. However, as the channeled particles travel further away from the lattice, suppression of Coulomb scattering and nuclear interactions takes place. In the case of Coulomb scattering, a form factor is calculated based on the classical minimal impact parameter related to the oscillation of the particle in the channel. It yields a rejection probability for the interaction which is a function of the momentum transfer, using a Fourier transform to go from one phase space to the other. In the case of nuclear scattering, the FLUKA cross-sections are tempered by a scaling factor representing the fraction of nuclear density integrated along the classical oscillatory path of the particle. Non-channeled particles, whose trajectory can come close to tangency with the curved crystal planes in the middle of the crystal, have the possibility to be captured in channeling or to be reflected by the planes and acquire a small kick in the direction opposite to the crystal bend, evaluated from Bondarenco's geometrical model.

In the second part of this report, an overview of the UA9 single-pass experimental activity in CERN North Area has been given. This experiment is the place where crystals are first tested and their characteristics measured before they can be considered as potential component of the SPS or LHC experimental crystal collimation layout. The experiment uses silicon strip detectors to reconstruct pairs of particle tracks upstream and downstream the crystal, yielding information about angular deflections imparted by the crystal.

In chapter 5, the comparison of this data with results of the simulation has been discussed. For experimental runs conducted between 2010 and 2012, from which several examples were treated, we have shown a quite good agreement in terms of channeling and dechanneling rates as well as in terms of angular distributions in the AM/VR and $\mathrm{CH}$ peaks. Simulated channeling rates differ by only few percents with respect to the experimental values. Other aspects of the angular distributions are reasonably reproduced, including VR/AM peak merging, dechanneling particles deflection profile or negative deflection offset of the VR/AM peak. On this last point, the accuracy of the kick seems improvable, with a discrepancy ranging from about +3 to almost $-2 \mu \mathrm{rad}$. The geometrical model applicability depends on the ratio between the crystal radius of curvature and the critical radius, here reaching limit values. 
Nuclear interaction reduction factors are consistent with expectations but have not been compared with the experiment. Indeed, the amount of nuclear interactions is not directly measured by the UA9 apparatus, and their precise estimation is still an open question among the collaboration. Similarly, lead ions runs can be handled by the code but no benchmarking could be performed due to the insufficient data collection.

Quasi-channeled particles are considered to behave like amorphous ones, except for their possibility of being captured or reflected during their crossing. In reality, it has been observed that quasi-channeled particles are resenting some of the crystal lattice symmetry and having different interaction rates than amorphous or channeled particles. This aspect can be improved by designing a separate treatment for quasi-channeled particles though, in the framework of crystal collimation, the description of channeling remains the main point of interest. This is only one of the perspectives of improvement for the future of the model. Several others can be pursued such as :

- The benchmarking of the event generator with $\mathrm{H} 8157 \mathrm{~A} \mathrm{GeV} / \mathrm{c}$ lead ions would be of critical interest to assess precisely the performance of the code in the case of a heavy ion beam.

- To obtain data about the nuclear interaction rates would allow to proceed to the benchmarking of the event generator in this respect.

- The implementation of a dedicated model of ionization for channeled particles.

- The treatment of ion electromagnetic dissociation in channeling.

- The channeling of negatively charged particles, that would require a different potential and revising the single scattering alteration.

- The incorporation of axial channeling into the model would make the tool more complete even though it is only marginally used in recent applications.

The model presented here accounts in a new and general way for coherent channeling events of positively charged particles. It paves the way for the development of a tool allowing critical energy deposition studies in a crystal environment, with the possibility of extending them to any synchrotron crystal-assisted collimation layout if used in conjunction with the FLUKA-SixTrack coupling. 



\section{APPENDIX A}

\section{IMPLEMENTATION DETAILED}

\section{A.1 Main function}

This appendix provides a slightly more technical description of the implementation. Four flowcharts explain the logic followed in the event generator. Key elements are the determination of channeling, the handling of interactions and of the tangency zone where volume effects occur. The core of the model (the main) is shown in figure A.1. For the sake of readability, three separated diagrams showing dedicated tasks are discussed in the following sections and shown in figs. A.2 to A.4.

The program starts with the initialization of beam and crystal parameters, taken from the input file, as well as the setting up of FLUKA media and geometry-related values. Upon entry in the crystal region, the transverse energy of the particle is calculated, which allows the determination of its regime (see section A.2, Channeling regime). If the particle is channeled, its direction is set to the channel one, the trajectory curvature is enabled and the amplitude of the dummy magnetic field used to reproduce the curvature is calculated.

The tracking of any particle in FLUKA is done by steps. For channeled and quasichanneled particles, processes that are normally handled in a continuous manner (MCS, ionization) are incorporated in the treatment of each individual step. The first stage is to determine the length of this step. For all particles, it is evaluated as the distance until the next interaction taking into consideration the cross-sections of all the involved processes. The particle is then transported along that step (along a curvy trajectory if the particle is channeled) and for quasi-channeled particles, the entry condition into the volume effects zone is verified. If applicable, volume effects-related operations are carried out (see section A.3). 


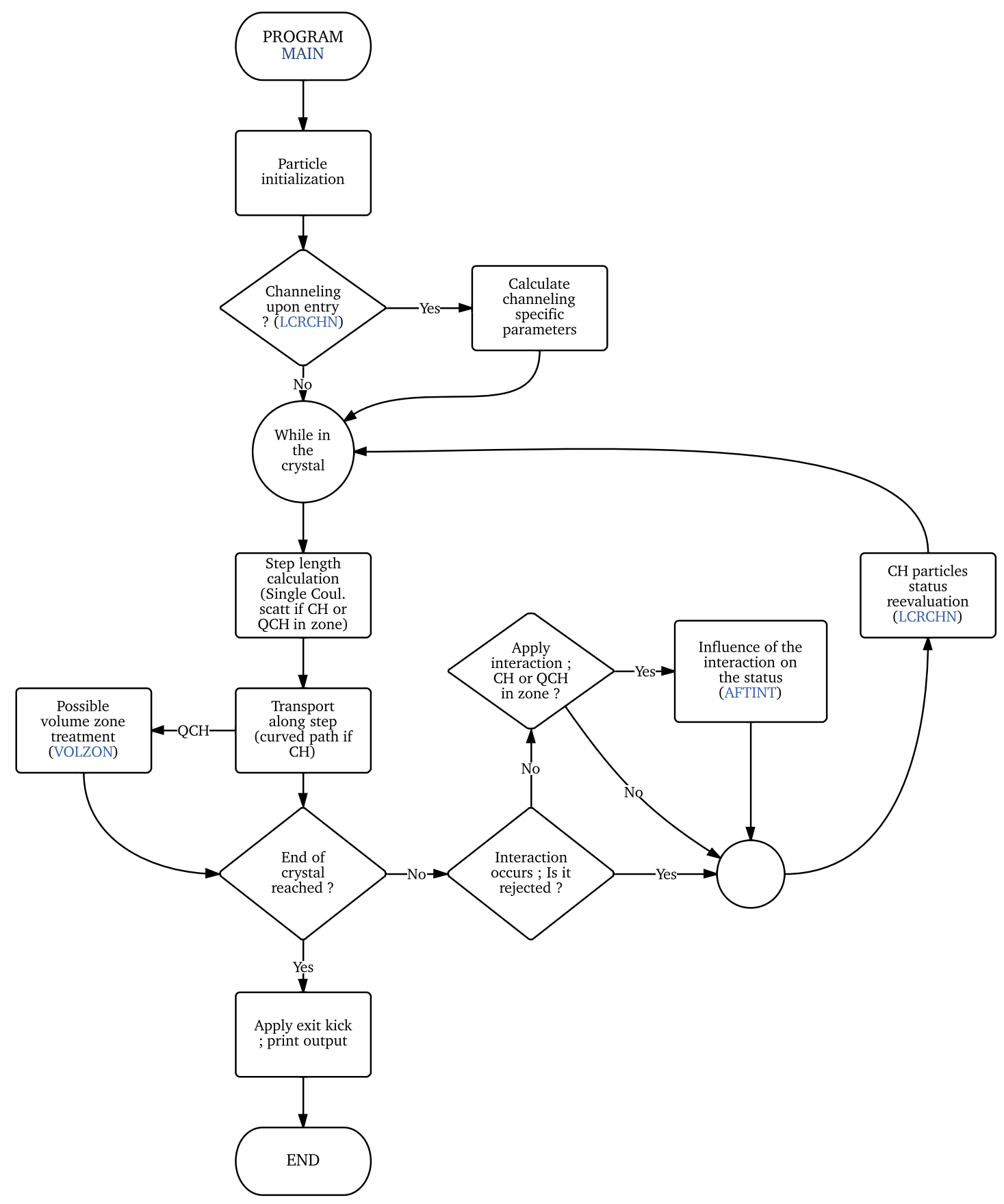

Fig. A.1. Flowchart representing the event generator main loop. Subroutines are indicated in blue and are detailed in the next sections. $\mathrm{CH}, \mathrm{QCH}$ and AM refer to particles in channeling, quasi-channeling and amorphous regimes respectively. 
If the particle does not exit the CRYSTAL region during the step, an interaction occurs whose type is determined based on the specific cross-sections of the phenomena. For channeled particles, single Coulomb scattering events involving large momentum transfers can be rejected depending on the oscillation amplitude of the particle (see form factor, p. 40. Channeled particles, as well as quasi-channeled particles in the volume effects zone, have their transverse energy modified by the interaction (see section A.4). The channeled particles trajectories, modified by the interaction, have then to be realigned with the channel direction and their status is reevaluated based on their new transverse energy value, possibly leading to capture or dechanneling. After this operation the particles enters a new step.

\section{A.2 Channeling regime determination}

This section explains the determination of the channeling status (AM, $\mathrm{CH}$ or QCH) of particles that are entering the crystal, or of those having undergone an interaction and thus a modification of their transverse energy. The corresponding flowchart is shown in fig. A.2.

The event generator is called as soon as a particle enters a region of the geometry flagged as CRYSTAL. When a particle enters the crystal, FLUKA recognizes the change of region. The channel orientation at the particle hit position corresponds to the vector $\boldsymbol{A}$ adjusted for torsion. The particle orientation in both transverse dimensions (along $\boldsymbol{A}$ and $\boldsymbol{B})$ are determined, taking care of using cross-products rather than dot-products. Indeed for angles used in channeling applications (few $\mu \mathrm{rad}$ ), the use of dot-products introduces operations on numbers very close to 1 leading to a critical loss of precision. The effective radius of curvature is then calculated. It differs from the nominal one for a crystal with torsion and depending on the particle motion component along $\boldsymbol{B}$ obtained before.

The next stage is the calculation of the particle transverse energy. This module is called upon entry in the region and when a particle is captured. It performs the following tasks :

- Moliere potential calculation with centrifugal component.

- Transverse position sampling (unless capture).

- Calculation of the potential and kinetic terms of the transverse energy which gives the total transverse energy.

- Determination of the potential well depth. 


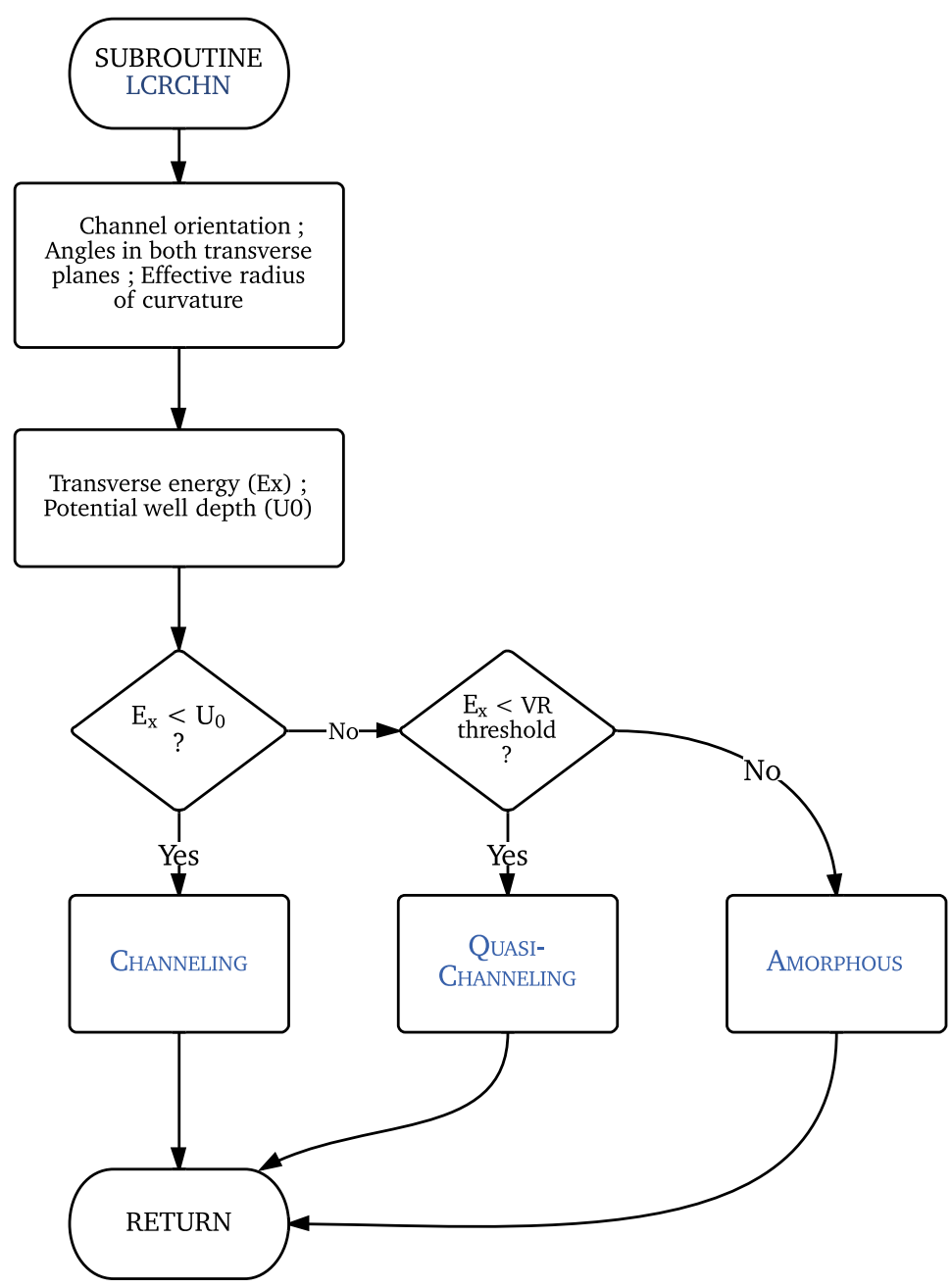

Fig. A.2. Flowchart illustrating how the status of a particle is (re)evaluated either upon entry in the crystal region or after an interaction, which modifies its transverse energy and can lead to dechanneling.

The transverse energy is compared with the thresholds defined in table 3.1 to determine of the particle is in channeling, quasi-channeling or amorphous mode. If it is channeled, the transverse energy value is stored for use in the dechanneling and interaction suppression determination (see sections 3.5 and 3.6).

\section{A.3 Volume effects zone}

Quasi-channeled particles propagate in the crystal in single-scattering mode. This means that, as for channeled particles, Coulomb scattering is evaluated step-by-step in- 


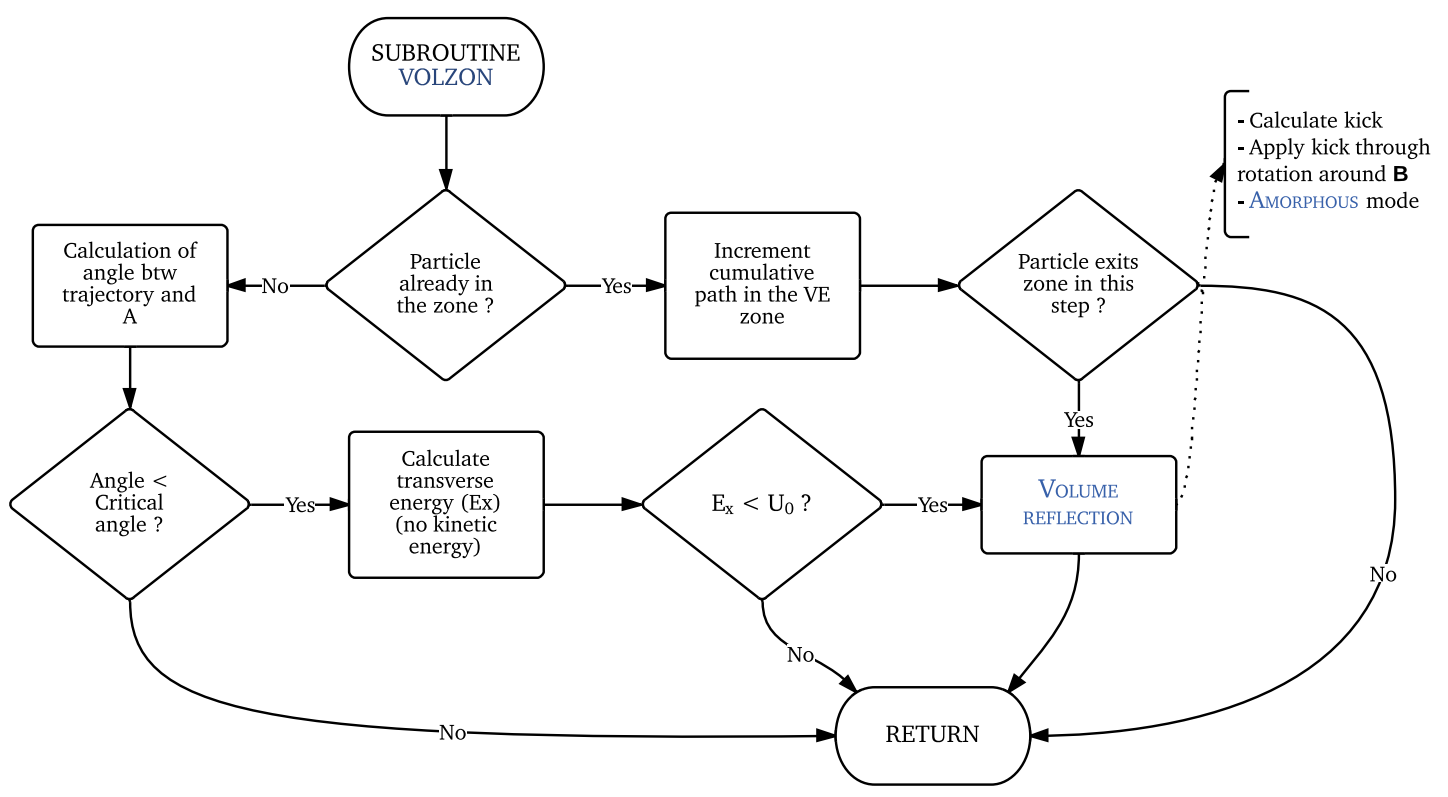

Fig. A.3. This flowchart shows how volume effects are implemented. Quasi-channeled particles enter the volume effects zone when their angle with the crystal planes gets smaller than the critical angle. At that point, their transverse energy is evaluated. If it is smaller than U0, volume reflection occurs. If in subsequent calls, the transverse energy is found to have dropped below $U_{0}$, the particle is captured. If not, the particle is reflected at the end of the zone. Upon return to the main, cumulative path and transverse energy are kept in memory and used in following steps. 
stead of the usual continuous MCS treatment. However, interaction rates and trajectories are the ones of amorphous mode particles.

At each step, the code verifies if a QCH particle trajectory is approaching tangency with respect to the crystal planes (angle smaller than $\theta_{c}$ ). If this is the case, the particle is attributed a transverse energy $E_{x}$ evaluated in a similar way as for channeled particles except that the kinetic term is considered to be 0 , corresponding to perfect tangency. If $E_{x}$ is smaller than $U_{0}$, the particle is reflected as it is considered to have been stopped by the previous potential barrier (imagine a particle of low transverse energy propagating to the right in fig. 2.9 and being reflected off of a lower energy channel). If not, the particle continues its way through the crystal, keeping $E_{x}$ value.

In subsequent steps, $E_{x}$ is reevaluated at each interaction, like for channeled particles (however close-collisions are not rejected in the case of QCH particles). As soon as this energy drops below $U_{0}$, the particle is captured. At the end of the zone, if $E_{x}$ has never dropped below $U_{0}$, the particle undergoes VR as it leaves the tangency region and propagate in the rest of the crystal in amorphous mode.

\section{A.4 After an interaction}

Figure A.4 sketches the steps conducted after an interaction for channeled particles as well as for quasi-channeled particles in the volume effects zone. The reevaluation of the transverse energy takes place at every step and gives an opportunity for dechanneling (respectively capture) of $\mathrm{CH}$ (resp. QCH) particles if $E_{x}$ goes above (resp. below) the potential barrier.

The particle direction vector before and after the interaction are normalized and projected on the plane perpendicular to $\boldsymbol{B}$. Then, the angle they form is translated to a transverse energy offset caused by the interaction. This contribution is considered to have $50 \%$ chance of increasing the transverse energy and $50 \%$ chance of decreasing it. The orientation of the channeled particle (only) is reset to the channel one. The interaction purpose is solely to modify $E_{x}$ and not change the trajectory. 


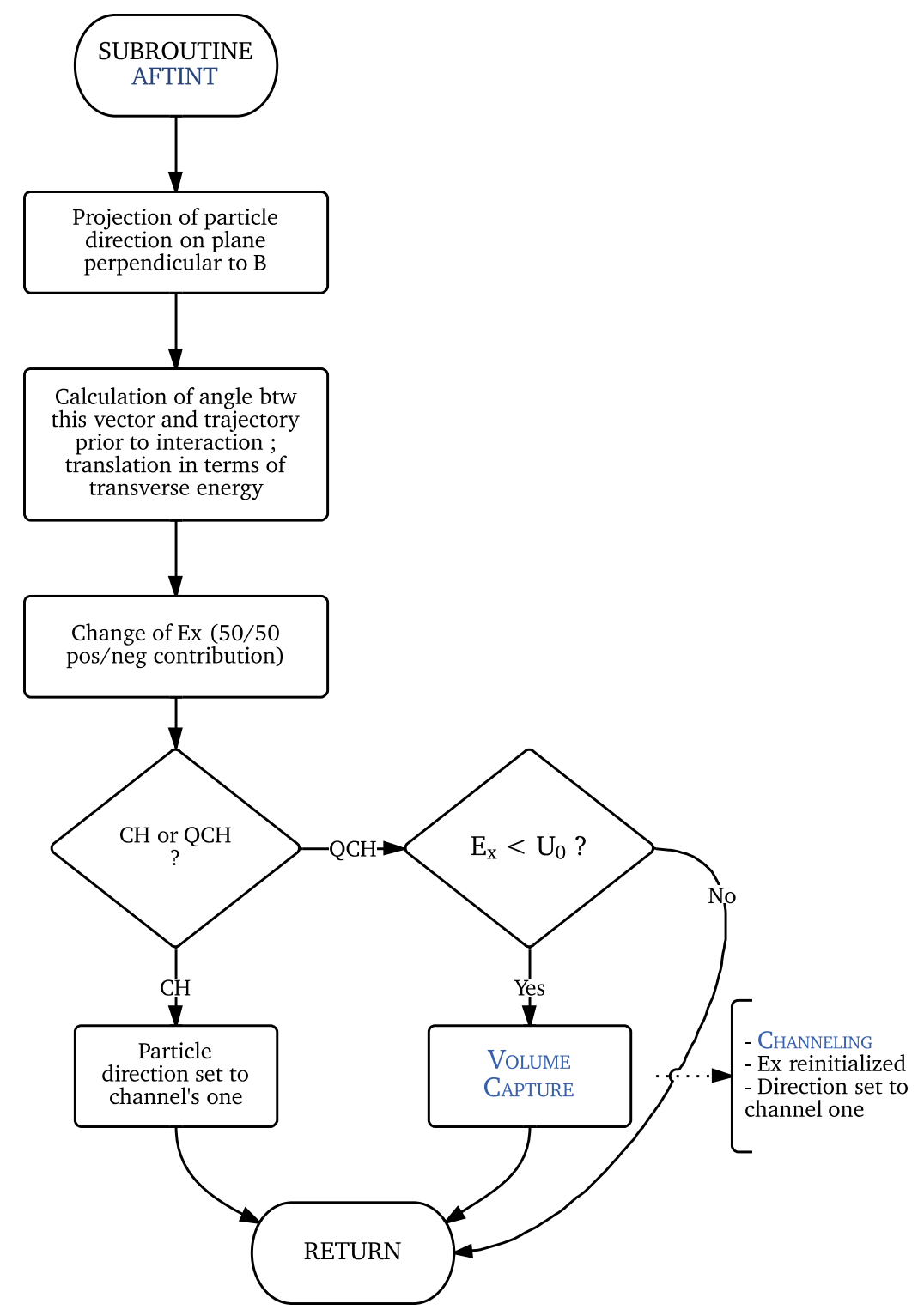

Fig. A.4. Transverse energy reevaluation of $\mathrm{CH}$ and QCH particles after an interaction. 



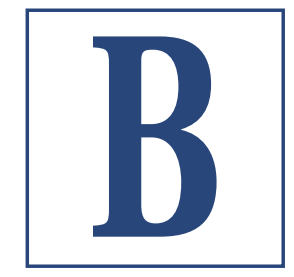

\section{APPENDIX B}

\section{CONFIDENCE INTERVALS}

The data analysis deals with three types of figures :

Means : positions in $x$ and $y$, total kick angle,

Standard deviations : beam divergence in $x^{\prime}$ and $y^{\prime}$, sigmas of the means given above,

Rates : channeling, dechanneling, VC,...

Errors bars are determined for the three types of data according to the standard results of statistics for a normal distribution with unknown $\mu$ and unknown $\sigma$ [67].

\section{B.1 Confidence interval on the mean}

Considering a population of interest with a normal distribution of unknown mean $\mu$ and variance $\sigma^{2}$ from which we have measured a sample $X_{1}, \cdots, X_{n}$ of mean $\bar{X}$ and variance $S^{2}$. The distribution of the random variable

$$
Y=\frac{\bar{X}-\mu}{\sigma \sqrt{n}}
$$

is normal. As the standard deviation is not known, it cannot be used to determine the confidence interval (CI). In reality, one has to use $S$ (which has some uncertainty) and to transform the normally-distributed variable $Y$ in a Student $t$-distributed $\hat{Y}$ variable

$$
\hat{Y}=\frac{\bar{X}-\mu}{S \sqrt{n}}
$$

For the sake of illustration, Student's distribution is shown in fig. B.1 where it is compared with a normal one. Student distribution tends to the normal distribution for large numbers of degrees of freedom. 


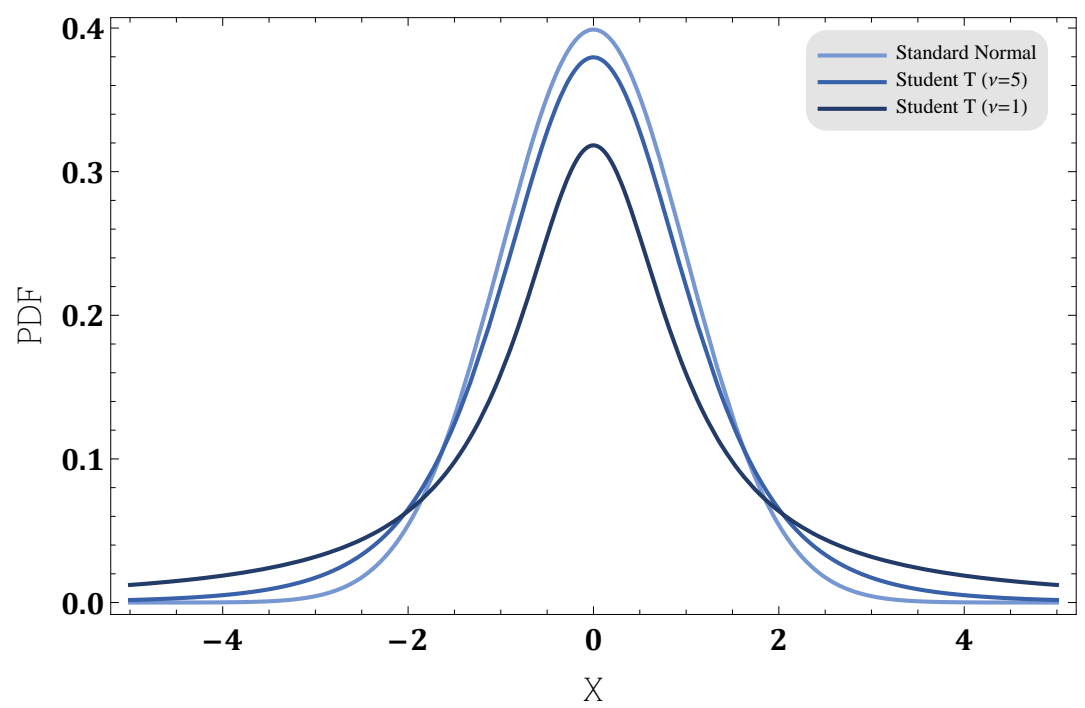

Fig. B.1. Student t-distribution with 1 and 5 degrees of freedom compared with the standard normal law. Student distribution has thicker tails and tends to be Gaussian for very large $v$.

In the case of crystal channeling data analysis, the number of degrees of freedom is as high as hundreds of thousands (number of events), and the distribution can be considered as normal. The CI corresponding to a confidence level of $100(1-\alpha) \%$ is then :

$$
\bar{X}-g_{\alpha / 2} \frac{S}{\sqrt{n}}<\mu<\bar{X}+g_{\alpha / 2} \frac{S}{\sqrt{n}}
$$

where $g_{\alpha}$ is the position of outer tail probability $\alpha$ in a normal distribution. The $\mathrm{CI}$ is, for large $n$, the same than in the case where the variance is known.

\section{B.2 Confidence interval on the variance}

The distribution of the measured variance $S$ normalized to the real variance $\sigma$

$$
X^{2}=\frac{(n-1) S^{2}}{\sigma^{2}}
$$

follows a chi-squared distribution with $(n-1)$ degrees of freedom ${ }^{1}$ (see fig. B.2). The interval corresponding to a confidence of $100(1-\alpha) \%$ is :

$$
S \sqrt{\frac{n-1}{\chi_{\alpha / 2, n}^{2}}}<\sigma<\sqrt{\frac{n-1}{\chi_{1-\alpha / 2, n}^{2}}}
$$

where $\chi_{\alpha / 2, n}^{2}\left(\chi_{1-\alpha / 2, n}^{2}\right)$ is the percentage point of the chi-squared distribution with $n$ degrees of freedom and a right (left) tail probability $\alpha / 2$.

\footnotetext{
${ }^{1}$ sum of squares of (n-1) normal standard random variables
} 


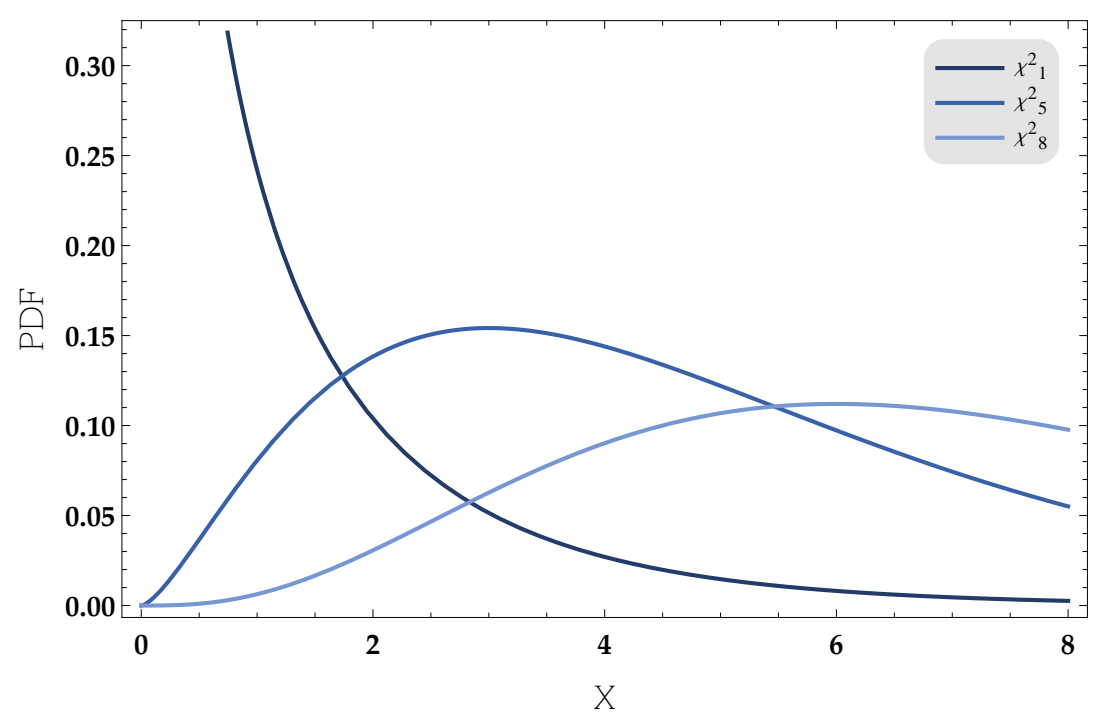

Fig. B.2. Examples of Chi-squared distributions for 1, 5 and 8 degrees of freedom. The variance of a normally distributed ensemble of samples with unknown mean and variance follows the chi-squared distribution with (n-1) degrees of freedom where $n$ is the number of samples in the population.

\section{B.3 Confidence interval for a population rate}

In a data analysis, the goal can also be to estimate the proportion of elements belonging to a class of interest. For example, the rate of particles having an exit kick larger than a given value. Let us consider a random sample of $n$ elements among which $m$ belong to our class of interest.

$$
\hat{p}=\frac{m}{n}
$$

is an estimator of the population proportion belonging to this class. The real proportion is distributed around $\hat{p}$ following a binomial distribution $(n, p)$. The mean and variance of such a distribution are :

$$
\begin{aligned}
\mu & =n \hat{p} \\
\sigma^{2} & =\frac{\hat{p}(1-\hat{p})}{n}
\end{aligned}
$$

When $n p \gg 1$, the distribution can be considered as normal. The CI becomes then :

$$
\hat{p}-g_{\alpha / 2} \sqrt{\frac{\hat{p}(1-\hat{p})}{n}}<p<\hat{p}+g_{\alpha / 2} \sqrt{\frac{\hat{p}(1-\hat{p})}{n}}
$$





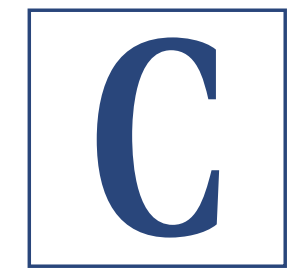

\section{APPENDIX C}

\section{ADDITIONAL COMPARISON WITH DATA}

In this appendix are presented additional angular deflection distributions corresponding to the 5 runs $608,630,899,1012$ and 1240 which have not been shown in chapter 5 . Information about the corresponding crystals, and the results of each run can be found in tables 5.6, 5.7 and 5.8.

The simulated horizontal deflection angle distributions are relatively close to the experiment. In the case of run 608 and 899, however, there is a non-negligible discrepancy at the level of the AM/VR peak. There, the distribution of reflected particles is somewhat

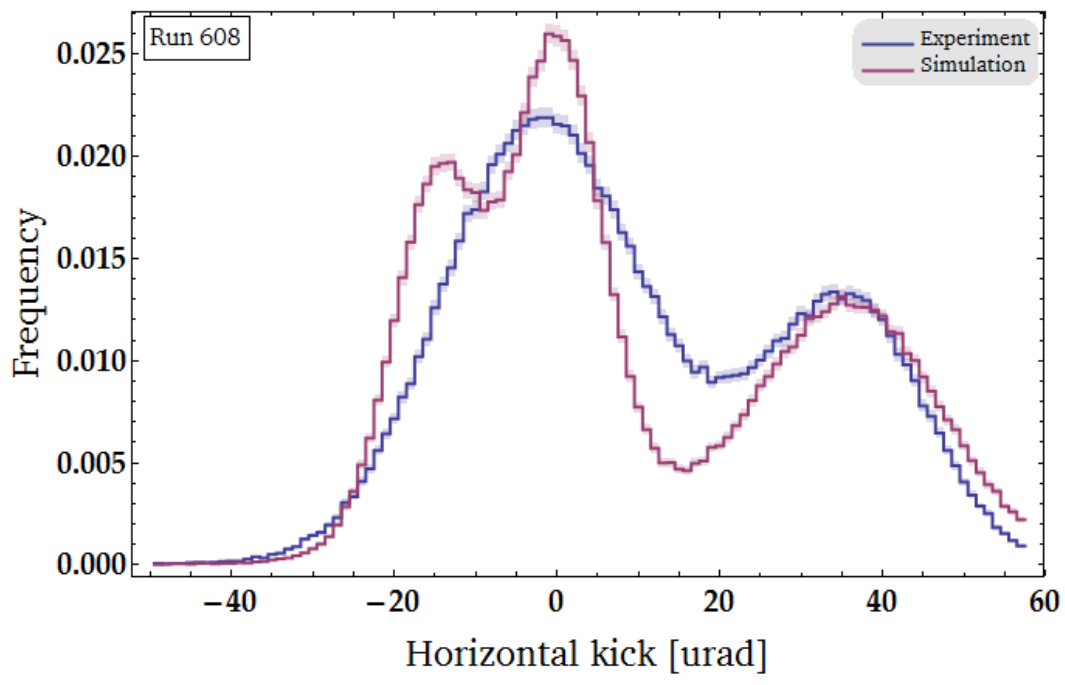

Fig. C.1. Angular deflection distributions for the experiment (blue) and the simulation (red) in channeling orientation for run 608 conducted with crystal STF 47. 


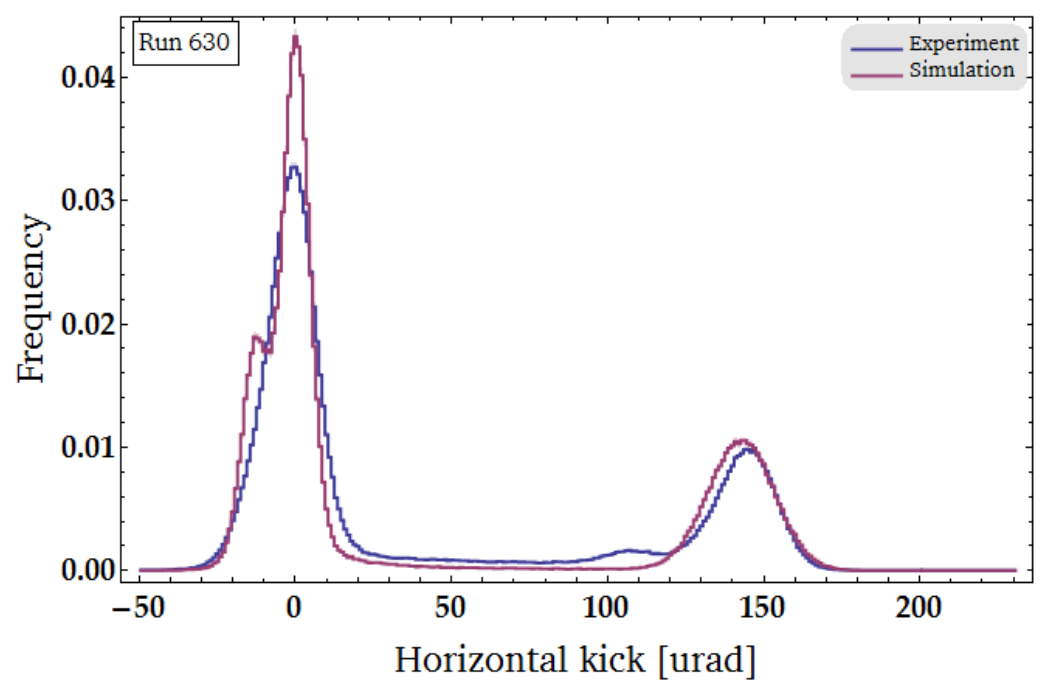

Fig. C.2. Angular deflection distributions for the experiment (blue) and the simulation (red) in channeling orientation for run 630 conducted with crystal STF 48.

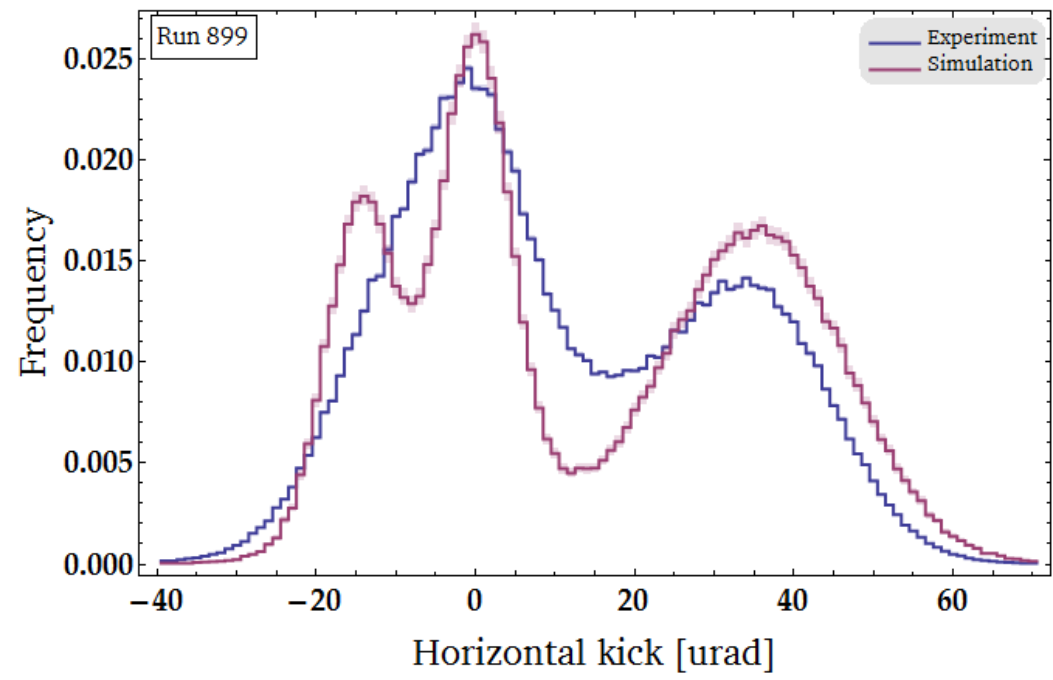

Fig. C.3. Angular deflection distributions for the experiment (blue) and the simulation (red) in channeling orientation for run 899 conducted with crystal STF 51. 


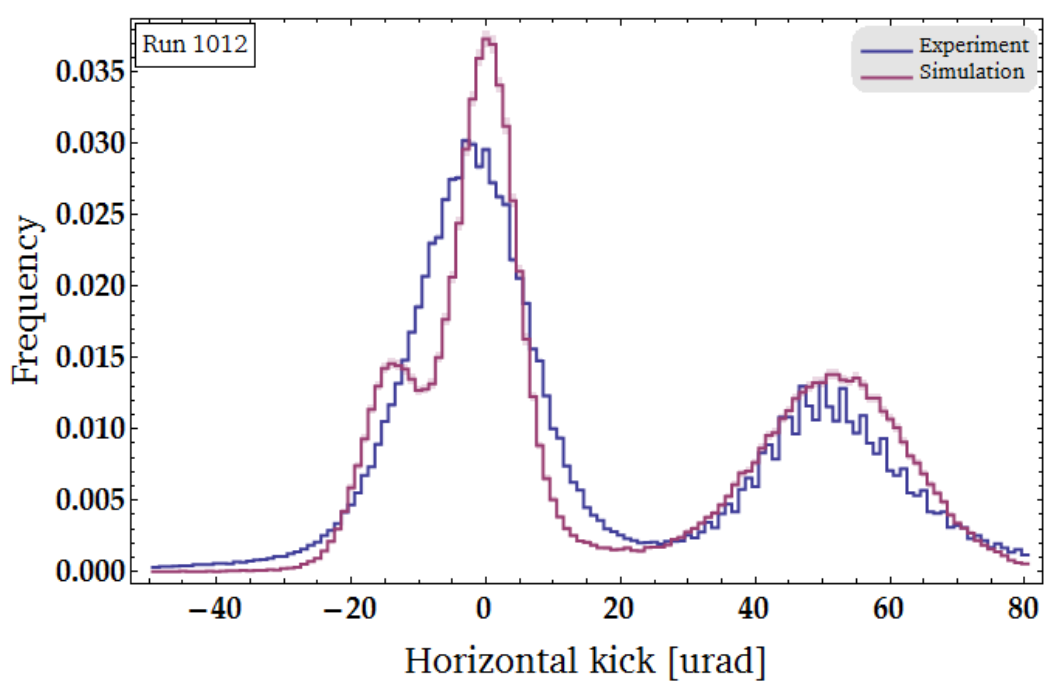

Fig. C.4. Angular deflection distributions for the experiment (blue) and the simulation (red) in channeling orientation for run 1012 conducted with crystal QMP 26.

detached from the amorphous mode particles one. The VR kick obtained from eq. (2.34) turns out to be quite large at $15 \mu \mathrm{rad}$, especially compared with the nominal bending angle of the crystal, at $35 \mu \mathrm{rad}$. As in the cases treated in the chapter 5 , the beam is almost perfectly centered on the crystal channels orientation and we expect VR and AM particles to represent comparable fractions of the total population.

This is not the case in the experiment. We can estimate the proportion of particles in both modes through a fit with a sum of two Gaussian distributions of same sigma and fixed means, but different amplitudes. The standard deviation is chosen to be the rms kick of the MCS process in the crystal. Also, the mean of the distributions is put at 0 , for the AM population and $-\theta_{v r}$, for the VR one. This fitting gives an estimate of the VR population around 4 times lower than the AM population whereas using the same method with the simulation results yields a factor 1.5 only. In addition to this imbalance, not properly reproduced by the simulation, the relatively high value of the VR kick larger than the MCS kick received in a silicon crystal of this thickness - is also a factor causing the peaks to be separated. 


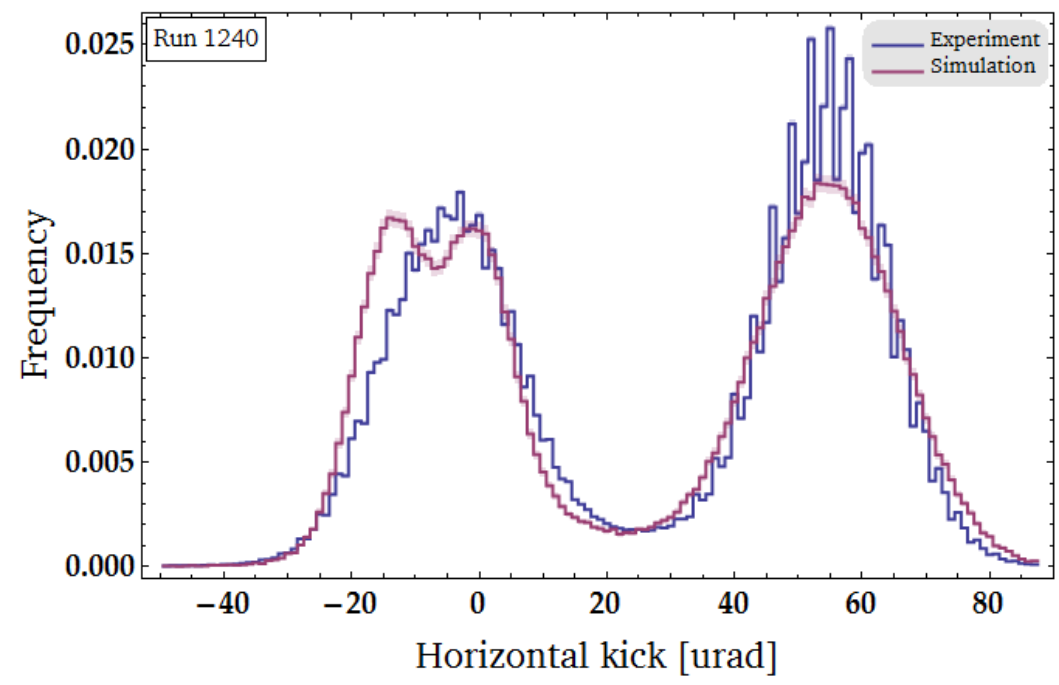

Fig. C.5. Angular deflection distributions for the experiment (blue) and the simulation (red) in channeling orientation for run 1240 conducted with crystal STF 70. 


\section{BIBLIOGRAPHY}

[1] W. SCANDALE et al., 'UA9 Report for 2011.' Technical Report CERN-SPSC-2011-037, CERN (2012).

[2] W. Scandale, G. ARduini, R. Assmann et al., 'Comparative results on Collimation of the SPS Beam of Protons and Pb Ions with Bent Crystals.' Physics Letters B, 703 (2011) 547 .

http://www.sciencedirect.com/science/article/pii/S0370269311009580

[3] G. Robert-Demolaize, Design and Performance Optimization of the LHC Collimation System. Ph.D. thesis, Université Joseph Fourier, Grenoble (2006).

[4] W. SCANDALE et al., 'LHC collimation with bent crystals - Letter of intent.' Technical Report CERN-LHCC-2011-007, CERN (2011).

[5] V. Previtali, Performance Evaluation of a Crystal-enhanced Collimation System for the LHC. Ph.D. thesis, Ecole Polytechnique Fédérale de Lausanne (2010).

[6] L. LARI, Beam-Machine Interaction Studies for the Phase II LHC Collimation System. Ph.D. thesis, Ecole polytechnique fédérale de Lausanne (2010).

[7] A. Lechner, M. Calviani, M. D. Castro et al., 'Robustness test of a silicon strip crystal for crystal-assisted collimation studies in the LHC.' In 'Proccedings of IPAC 2013,' IPAC 2013.

[8] N. Ashcroft, I. Mermin and N. David, Solid State Physics. Brooks/Cole (1976), ISBN 0-03-08993-9.

[9] 'Wikipedia Commons.'

http://upload.wikimedia.org/wikipedia/commons/

[10] V. M. Biryukov, Y. A. Chesnokov and V. I. Kotov, Crystal Channeling and its Application at High-Energy Accelerators. Springer (1997), ISBN 3-540-60769-2.

[11] J. LindHARD, 'Influence of Crystal Lattice on Motion of Energetic Charged Particles.' Matematisk-Fysiske Meddelelser Kon. Dan. Vid. Selskab, 34 (1965) 1.

[12] G. Moliere, 'Theorie der Streuung schneller geladener Teilchen I : Einzelstreuung am abgeschirmten Coulomb-Feld.' Zeitschrift für Naturforschung, A (1947) 133.

[13] G. Diambrini Palazzi, 'High-Energy Bremsstrahlung and Electron Pair Production in Thin Crystals.' Review of Modern Physics, 40 (1968) 611.

[14] P. Flubacher, A. Leadbetter and J. Morrison, 'Heat Capacity of Pure Silicon and Germanium.' Philosophical Magazine, 4 (1959) 273. 
[15] M. Bondarenco, 'Model Solution for Volume Reflection of Relativistic Particles in a Bent Crystal.' Preprint : Arxiv:0911.0107, (2010).

[16] W. Scandale, D. A. Still, A. Carnera et al., 'High-efficiency Volume Reflection of an Ultrarelativistic Proton Beam with a Bent Silicon Crystal.' Physical Review Letters, 98 (2007) 154801.

http://link.aps.org/doi/10.1103/PhysRevLett.98.154801

[17] J. STARK and G. WENDT, 'Über das Eindringen von Kanalstrahlen in feste Körper.' Annalen der Physik, 38 (1912) 921.

[18] J. A. Davies, J. D. Mcintyre, R. L. Cushing and M. Lounsbury, 'The Range of Alkali Metal Ions of keV Energies in Aluminum.' Canadian Journal of Chemistry, 38 (1960) 1535.

http://www.nrcresearchpress.com/doi/abs/10.1139/v60-214

[19] J. A. Davies, J. Friesen and J. D. McIntyre, 'A Radiochemical Technique for studying Range Energy Relationships for Heavy Ions of keV Energies in Aluminum.' Canadian Journal of Chemistry, 38 (1960) 1526.

[20] M. W. Thompson, 'A Numerical Correction to "The Penetration of Energetic Ions through the Open Channels in a Crystal Lattice" by R. S. Nelson and M. W. Thompson, Phil. Mag., 8 (1963), 1677.' Philosophical Magazine, 9 (1964) 1069. http://www.tandfonline.com/doi/abs/10.1080/14786436408211917

[21] C. Lehmann and G. LeIBFRIED, 'Long-Range Channeling Effects in Irradiated Crystals.' Journal of Applied Physics, 34 (1963) 2821.

http://link. aip.org/link/?JAP/34/2821/1

[22] J. LindhARD, 'Motion of Swift Charged Particles, as Influenced by Strings of Atoms in Crystals.' Physics Letters, 12 (1964) 126.

[23] D. ERginsoy, 'Anisotropic Effects in Interactions of Energetic Charged Particles in a Crystal Lattice.' Physical Review Letters, 15 (1965) 360.

http://link.aps.org/doi/10.1103/PhysRevLett.15.360

[24] D. S. Gemmell and R. E. Holland, 'Blocking Effects in the Emergence of Charged Particles from Single Crystals.' Physical Review Letters, 14 (1965) 945.

http://link.aps.org/doi/10.1103/PhysRevLett.14.945

[25] A. Tulinov, V. Kulikauskas and M. Malov, 'Proton Scattering from a Tungsten Single Crystal.' Physics Letters, 18 (1965) 304 . http://www.sciencedirect.com/science/article/pii/0031916365903495

[26] O. Fich, J. Golovchenko, K. Nielsen et al., 'Channeling of $1.1 \mathrm{GeV} / \mathrm{c}$ Protons and Pions.' Physics Letters B, 57 (1975) 90 .

http://www.sciencedirect.com/science/article/pii/0370269375902518 
[27] E. Tsyganov, 'Some Aspects of the Mecanism of a Charged Particle Penetration through a Monocrystal.' Technical Report TM-682, Fermi National Accelerator Laboratory (1976).

[28] A. Elishev, N. Filatova, V. Golovatyuk et al., 'Steering of Charged Particle Trajectories by a Bent Crystal.' Physics Letters B, 88 (1979) 387 . http://www.sciencedirect.com/science/article/pii/0370269379904921

[29] J. Bak, G. Melchart, E. Uggerøj et al., 'Bending of High Energy Beams Using Axial and Planar Channeling.' Physics Letters B, 93 (1980) 505 . http://www.sciencedirect.com/science/article/pii/0370269380903767

[30] V. M. Biryukov, V. I. Kotov and Y. A. Chesnokov, 'Steering of high-energy charged-particle beams by bent single crystals.' Physics-Uspekhi, 37 (1994) 937. http://stacks. iop.org/1063-7869/37/i=10/a=R01

[31] J. BAK, P. Jensen, H. MADsbøll et al., 'Detailed Investigation of the Channeling Phenomena Involved in Bending of High-Energy Beams by Means of Crystals.' Nuclear Physics B, 242 (1984) 1 .

http://www.sciencedirect.com/science/article/pii/0550321384901305

[32] W. Gibson, I. Kim, M. Pisharody et al., 'Deflection of High Energy Channeled Charged Particles by Elastically Bent Silicon Single Crystals.' Nuclear Instruments and Methods in Physics Research B, 2 (1984) 54.

http://www.sciencedirect.com/science/article/pii/0168583X8490154X

[33] V. A. Andreev, V. V. Baublis, E. A. Damaskinski et al., 'Experimental Observation of Volume Capture by a Curved Single Crystal in the Channeling Regime.' JETP Letters, 36 (1982) 340 . http://www.jetpletters.ac.ru/ps/1337/article_20196.shtml

[34] A. TARATin and S. Vorobiev, 'Volume reflection of high-energy charged particles in quasi-channeling states in bent crystals.' Physics Letters A, 119 (1987) 425 . http://www. sciencedirect.com/science/article/pii/0375960187905871

[35] H. Akbari, X. Altuna, S. Bardin et al., 'First results on proton extraction from the CERN-SPS with a bent crystal.' Physics Letters B, 313 (1993) 491 . http://www.sciencedirect.com/science/article/pii/037026939390024C

[36] K. Elsener, G. Fidecaro, M. Gyr et al., 'What did we learn from the Extraction Experiments with Bent Crystals at the CERN SPS ?' In 'Proceedings of the EPAC-98,' EPAC98.

[37] A. G. Afonin, V. T. BARAnov, V. M. Biryukov et al., 'High-Efficiency Beam Extraction and Collimation Using Channeling in Very Short Bent Crystals.' Physical Review Letters, 87 (2001) 094802.

http://link.aps.org/doi/10.1103/PhysRevLett.87.094802 
[38] R. P. F. III, A. Drees, D. GAssner et al., 'RHIC Crystal Collimation.' Nuclear Instruments and Methods in Physics Research B, 234 (2005) 47. http://www.sciencedirect.com/science/article/pii/S0168583X05002260

[39] R. CARRIgAN, 'Channeling Collimation Studies at the Fermilab Tevatron.' Technical Report Fermilab-CONF-06-309-AD, FNAL (2006).

[40] W. SCANDALE et al., 'UA9 Report for 2012.' Technical Report CERN-SPSC-2012-033, CERN (2012).

[41] A. Ferrari, P. R. SAla, A. FAsso and J. RANFt, 'FLUKA: a Multi-Particle Transport Code.' Technical Report CERN-2005-10, CERN (2005).

[42] G. Battistoni, S. Muraro, P. R. Sala et al., 'The FlUKA Code : Description and Benchmarking.' In 'Proceedings of the Hadronic Shower Simulation Workshop 2006,' HSS06, pages 31-49.

[43] F. Schмidt, 'SixTrack : User’s Reference Manual.' (2010).

[44] N. HolDEN, 'Development of the ICOSIM program and application to magnetised collimators in the LHC.' Technical Report AB-Note-2008-054, CERN (2008).

[45] D. Mirarchi, W. Scandale, S. Redaelli and V. Previtali, 'Layouts for Crystal Collimation Tests at the LHC.' In 'Proceedings of IPAC 2013,' page 966.

[46] V. BiRYukov, 'Crystal Channeling Simulation CATCH 1.4 : User Guide.' (2001).

[47] A. TARATIN, 'Computer Simulation of Accelerator Beam Extraction with a Bent Crystal.' Nuclear Instruments ans Methods in Physics Research B, 95 (1995) 243 .

[48] A. Mereghetti, F. Cerutti, R. De Maria et al., 'SixTrack-Fluka Active Coupling for the Upgrade of the SPS.' In 'Proceedings of IPAC 2013,' .

[49] 'FLUKA official website.'

www.fluka.org/

[50] A. Ferrari, P. R. Sala, R. Guaraldi and F. Padoani, 'An Improved Multiple Scattering Model for Charged Particle Transport.' Nuclear Instruments and Methods in Physics Research B, 71 (1992) 412.

[51] J. LEWIN and P. SMith, 'Review of Mathematics, Numerical Factors, and Corrections for Dark Matter Experiments based on Elastic Nuclear Recoil.' Astroparticle Physics, 6 (1996) 87 .

http://www.sciencedirect.com/science/article/pii/S0927650596000473

[52] U. FANO, 'Inelastic Collisions and the Moliere Theory of Multiple Scattering.' Physical Review, 93 (1954) 117. 
[53] B. R. Appleton, C. Erginsoy and W. M. Gibson, 'Channeling Effects in the Energy Loss of 3-11-MeV Protons in Silicon and Germanium Single Crystals.' Physical Review, 161 (1967) 330.

http://link.aps.org/doi/10.1103/PhysRev.161.330

[54] H. Esbensen and J. Golovchenko, 'Energy Loss of Fast Channeled Particles.' Nuclear Physics A, 298 (1978) 382.

[55] W. Scandale, R. Losito, M. Silari et al., 'Probability of inelastic nuclear interactions of high-energy protons in a bent crystal.' Nuclear Instruments and Methods in Physics Research B, 268 (2010) 2655.

http://www.sciencedirect.com/science/article/pii/S0168583X1000635X

[56] C. PAJAReS and Y. M. ShabelSKI, Relativistic Nuclear Interactions. Editorial URSS (2007).

[57] A. Mazzolari, Manipulation of Charged Particle Beams through Coherent Interactions with Crystals. Ph.D. thesis, Univeristà degli Studi di Ferrara (2009).

[58] S. Timoshenko and J. Goodier, Theory of Elasticity. McGraw-Hill Book Company, 2 edition (1951).

[59] J. J. Wortman and R. A. Evans, 'Young's Modulus, Shear Modulus, and Poisson's Ratio in Silicon and Germanium.' Journal of Applied Physics, 36 (1965) 153. http://link. aip.org/link/?JAP/36/153/1

[60] Y. Ivanov, A. Petrunin and V. Skorobogatov, 'Observation of the elastic quasimosaicity effect in bent silicon single crystals.' JETP Letters, 81 (2005) 99. http://dx.doi.org/10.1134/1.1897998

[61] Y. AdischeV, S. AfANASIEV, V. Boiko et al., 'First observation of parametric X-rays produced by moderate relativistic protons and carbon nuclei in Si crystals.' Nuclear Instruments and Methods in Physics Research B, 252 (2006) 111. http://www.sciencedirect.com/science/article/pii/S0168583X0600855X

[62] W. Scandale, G. Arduini, R. Assmann et al., 'Observation of parametric X-rays produced by $400 \mathrm{GeV} / \mathrm{c}$ Protons in bent Crystals.' Physics Letters B, 701 (2011) 180 http://www.sciencedirect.com/science/article/pii/S0370269311005910

[63] W. Scandale, A. Vomiero, E. Bagli et al., 'Multiple volume reflections of highenergy Protons in a Sequence of bent Silicon Crystals assisted by Volume Capture.' Physics Letters B, 688 (2010) 284. http://www.sciencedirect.com/science/article/pii/S0370269310005071 
[64] M. Pesaresi, W. Ferguson, J. Fulcher et al., 'Design and Performance of a highrate, high angular Resolution Beam Telescope used for Crystal Channeling Studies.' Journal of Instrumentation, 6 (2011) P04006.

[65] M. Pentia, G. Iorgovan and A. Minul, 'Multiple Scattering Error Propagation in Particle Track Reconstruction.' Preprint : arxiv.org/abs/hep-ex/9406006, (1994).

[66] M. French, L. Jones, Q. Morrissey et al., 'Design and results from the APV25, a deep sub-micron CMOS front-end chip for the CMS tracker.' Nuclear Instruments and Methods in Physics Research A, 466 (2001) 359. http://www.sciencedirect.com/science/article/pii/S0168900201005897

[67] D. Montgomery and G. Runger, Applied Statistics and Probability for Engineers. John Wiley and Sons, 5th edition (2010), ISBN 978-0-470-05304-1. 